\title{
SOLUTION OF THE PLATEAU PROBLEM FOR $m$-DIMENSIONAL SURFACES OF VARYING TOPOLOGICAL TYPE
}

\author{
BY \\ E. R. REIFENBERG \\ Bristol
}

\section{Introduction}

The Plateau Problem consists in showing that the greatest lower bound of the areas of surfaces with a given boundary is attained. This depends primarily on the meaning we attach to the word surface. In the classical conception we start with a two-dimensional manifold $R$ with boundary $C$ and a set $A$ homeomorphic to $C$; we then say that $S$ is a surface of class $\mathcal{G}_{R}$ with boundary $A$ if there is a continuous mapping of $R$ onto $S$ which maps $C$ onto $A(1-1)$ and bicontinuously. In this sense the problem was very elegantly solved by Jesse Douglas in the 1930's [5].

But this solution leaves the question incomplete in a number of important respects. In the first place the problem so posed deals only with a class of surfaces which are all of the same topological type and for each topological type we have a separate theorem. Now if we are prepared to consider as a surface any set $S$ which is a mapping of a manifold $R$ whose boundary is homeomorphic to $A$, then surely we should be interested in the class of all such $S$ where $R$ is allowed to vary. When dealing with the problem in this light it seems intuitive that a minimum will be attained provided we admit as surfaces sets which, while they are manifolds at points away from the boundary, will, when the boundary is complicated, have infinitely many loops and infinite connectivity near the boundary. However this involves a comparison between surfaces which are not mappings of a common base space, and the classical methods are inherently very ill adapted for this. In the second place while it is intuitive that any set which is a surface of minimum area in some sense will be locally well-behaved, this is a result which it would be nice to prove and this cannot be done if we only consider locally well-behaved surfaces in the first place. In other

1-60173032, Acta mathematica. 104. Imprimé le XX 21 septembre 1960 
words it would be nice to investigate the structure of sets of minimum area without prejudging the issue by assuming that the sets are manifold like.

Finally the classical solution dealt only with the two-variable case. A great deal is known about functions of two variables particularly about harmonic and conformal functions and these are particularly relevant to this problem; on the other hand little is known about functions of $m$ variables and hence the study of the Plateau problem for $m$-dimensional surfaces was beyond the scope of the classical methods.

We considered that a possibly fruitful approach might be to consider as a surface any closed set $S$ containing the boundary $A$ provided only that it satisfied some topological condition ensuring that it "spanned the holes in $A$ ". If we could prove that the minimum area was attained in such a wide class we might then go further and investigate what sort of structure a minimum area surface must have. This programme has been carried out in the present work. The reader should note that once we have proved that the surface of minimum area is locally Euclidean then in the two-dimensional case we link up with the classical theory established by Lebesgue, Tonelli, Rado, Douglas, Morrey, McShane, and others, to whose papers the reader is referred. For [10] if a manifold is of minimum area in the sense of Hausdorff measure (which we use) then it is so also in the sense of Lebesgue area and hence (e.g. [12]) it is a minimal surface in the sense of differential geometry. Of course for three or higher dimensional suriaces this still leaves an important problem.

However to get back to the question of defining a surface, so far we have merely replaced the question of what we mean by saying " $S$ is a surface" by the question of what we mean by " $S$ spans the holes in $A$ ". But it is precisely to answer this last question that algebraic topology was invented and I was very fortunate in having from the start the co-operation of Dr. J. F. Adams who devised suitable definitions of a surface and wrote an appendix to this paper proving that his definitions had the intuitively plausible properties which would permit us to cut holes in surfaces and patch these again by other surfaces and generally indulge in natural geometrical constructions. He also devised a multitude of ingenious examples both to illustrate his definitions and to show their limitations; these also are given in his appendix. I should also like to take this opportunity of thanking Dr. H. B. Griffiths without whose presence in Bristol the writing up of this paper might have suffered a great deal more, from the fact that Dr. Adams was 150 miles away in Cambridge. The methods of algebraic topology are powerful by their abstraction and generality and this implies as usual that they are not easily visualized by the non-specialist. I have no doubt that our definitions will be generally accepted as 
reasonable but it seemed desirable to state two special cases which can be deduced from our general theorems and which can be stated in geometrically plausible terms. They do in fact, for this very reason, cover what $I$ feel are the most interesting results of this paper.

The reader will have noticed that I have not discussed the meaning of area. All reasonable definitions of area should have the same value on Minimal surfaces and so the choice does not seem inherently important. One could define what is an equivalent of Lebesgue area for arbitrary sets and I shall discuss this in the section on unsolved problems, but since we are dealing with the problem from a set theoretic point of view it seems sensible to use a measure rather than an area. Hausdorff spherical measure-first introduced into this field by A. S. Besicovitch [3, 4]-has proved a powerful tool and I have chosen it. The main justification for this choice lies $I$ feel in the existence of this paper.

In order to state the first of the geometrical theorems mentioned above we will have to talk about one boundary $B$ being "near" to another boundary $A$. To say merely that $B$ lies in a neighbourhood of $A$ is altogether too crude and cannot be enough; on the other hand, since in our case we cannot demand that $A$ and $B$ are homeomorphic the Frechet concept of distance is not applicable either. The following definition meets the case:-If $A$ and $B$ are two finite sets of disjoint simple closed Jordan curves then $B$ is said to be near $A$ if there exists a manifold $R$ (which may be one-sided or two-sided) and a classical surface $S \in \mathcal{G}_{R}$ with boundary $A+B$ such that the whole of $S$ lies near $A$. Suppose now that given a finite set $A$ of simple closed disjoint Jordan curves (which may be knotted or interlinked in any manner) in three-dimensional Euclidean space, we define $S$ to be a surface of class $\mathcal{G}$ with boundary $A$ if it is the sum of a monotone increasing sequence of manifolds whose boundaries tend to $A$. Then we have

THEOREM 1. The minimum area in $\mathcal{G}$ is attained.

As for the second of our "special case theorems", suppose that $A$ is a homeomorph of an $(m-1)$-dimensional sphere surface in $n$-dimensional Euclidean spacein other words a topological $m-1$ sphere. We say that a set $S$ is a surface of class $\mathcal{G}^{*}$ with boundary $A$ if $S$ is closed and contains $A$ while there is no continuous mapping of $S$ into $A$ which maps each point of $A$ onto itself-that is if $A$ is not a retract of $S$. If $S$ is a surface in this sense and there are no proper subsets of $S$ which are surfaces with boundary $A$ then we say that $S$ is a proper surface. We then have 


\section{THEOREM 2.}

i) The minimum area in $\mathcal{G}^{*}$ is attained.

ii) Every surface in $\mathcal{G}^{*}$ contains a proper surface.

iii) Every proper surface of minimum area in $\mathcal{G}^{*}$ has positive m-dimensional Hausdorff measure and is locally Euclidean of dimension $m$ at almost all points not belonging to $A$.

Theorem 1 solves the problem of finding a surface of minimum area in a class containing all manifolds. Theorem 2 is interesting not only because it is the first case of a solution of the Plateau problem for $m$ dimensional surfaces $(m>2)$ but also because of the extreme weakness of the definition of a surface together with the great strength of conclusion (iii). Example 7 of the Appendix gives a proper two-dimensional surface of minimum area of class $\mathcal{G}^{*}$ whose boundary is a simple closed Jordan curve but which does not belong to $\mathcal{G}$ and has a line along which it is not locally Euclidean. Examples 8 and 9 of the Appendix however show that even $\mathcal{G}^{*}$ is not exhaustive and that there are sets not belonging to it which we might well wish to call a surface. The matter is dealt with further in the section on unsolved problems,

I shall next state the theorem of which Theorems 1 and 2 are special cases. First I will define a surface; this definition is given again with more detail and some discussion in Dr. Adams' appendix.

Definition. Let $G$ be a compact Abelian group. Let $S$ be a closed set in n-dimensional Euclidean space and $\boldsymbol{A}$ a closed subset of $S$. Let $m$ be a non-negative integer. Then there is defined the Cech homology group $H_{m}(S, A ; G)$; if $A$ is empty this is written $H_{m}(S ; G)$. Let $K$ be the kernel of the inclusion homomorphism

$$
i_{*}: H_{m-1}(A ; G) \rightarrow H_{m-1}(S ; G)
$$

Let $L$ be any subgroup of $H_{m-1}(A ; G)$. Then we say that $S$ is a surface of class $\mathcal{G}^{G}$ with boundary $\supset L$ if $K \supset L$. Moreover if $S$ is a surface in the above sense but there are no closed proper subsets of $S$ containing $A$ which are surfaces with boundary $\supset L$ then $S$ is said to be a proper surface.

It will be proved that every surface contains a proper surface. Let $W_{m}$ be the volume of an $m$-dimensional solid Euclidean unit sphere, and let $\Lambda^{m}$ dencte Hausdorff spherical $m$-dimensional measure.

MAIN THEOREM. The minimum area of surfaces of class $\mathcal{G}^{G}$ with boundary $\supset L$ is attained and if $S$ is a proper surface of minimum area then $S$ will be locally 
Euclidean at all non-boundary points at which the lower density does not exceed one, that is at almost all non-boundary points. Moreover if $P \in S-A$ and $S(P, r)$ is a sphere not meeiing $A$ then $\Lambda^{m} S S(P, r) \geqslant W_{m} r^{m}$.

It will be shown that theorem I follows from this by taking $G$ to be the group of integers modulo 2 and that theorem 2 follows from the case where $G$ is the group of real numbers modulo 1 .

The proof of the main theorem is long and intricate--indeed it takes up almost the whole rest of the paper--I am therefore going now first to give the main structure and general ideas of the proof without any attempt at rigour or precision. I shall use the language of the case $m-2, n=3$.

In the first place the class of closed sets is known to form a locally compact metric space with the obvious distance function $d(X, Y)=-\underset{x \in X}{\operatorname{Max}} d(x, Y) \div \underset{y \in Y}{\operatorname{Max}} d(y, X)$. The topological condition we impose on a set to make it a surface is preserved under convergence in this space provided the boundary is kept fixed and hence any bounded class of surfaces with a given boundary is compact. Thus in order to prove the existence theorem we need only find a sequence of surfaces whose areas tend to the minimum and such that the convergence is lower semi-continuous in area. Now we cannot just take any sequence because a surface may have long thin tentacles which contribute little to the area but result in the limit set containing a lot of unnecessary points. In order to cut out the possibility of such ientacles we divide space into cubes and amongst our surfaces of area near the minimum we select the one which meets the least number of such cubes. To obtain a sequence of surfaces we take the cubes of finer and finer mesh. The fact that this will cut out the tentacles is plausible but the question is how to express it. Suppose $A(P, r)$ is the area of the part of the surface inside a sphere $S\left(P^{\prime}, r\right)$ of centre $P$ and radius $r$ and that $l(P, r)$ is the length of the intersection with the sphere surface then at the end of a tentacle $\mathrm{A}(P, r) / r^{2}$ will be small for small $r$ and we must therefore show that under the above construction $A(P, r) / r^{2}$ will be bounded below whenever $r$ is larger than the cube mesh.

In the Appendix Dr. Adams proves various theorems of the kind that if we cut a hole in a surface and replace it with another surface then we still have a surface. In Chapter I I utilise these to construct a number of sets having suitable epiperimetric properties which are then available to patch holes in surfaces in this way. The epiperimetric inequalities used are of two types, the first is $A<k l^{2}$ where $A$ is the area of the construct and $l$ the length of its boundary (in the case $m=2$ ). From this we 
obtain $\int l(P, r) d r<A(P, r)<k l^{2}(P, r)$ whence we can prove that $l(P, r)>r / 2 k$ and $A(P, r)>r^{2} / k$.

The second type of inequality is $A<\frac{1}{2} l r$ from which we can prove that $A(P, r) / r^{2}$ is increasing.

In Chapter 2 we use these techniques to obtain a sequence $S_{n}$ of surfaces whose areas tend to the minimum and with the property that $\phi(P, r)=\lim _{n \rightarrow \infty} A_{n}(P, r) / r^{2}$ is bounded below for all $P$ of the limit surface $S$ and all $r$ such that $P$ is further than $r$ from the boundary. This incidentally implies that $S$ has finite area. We consider this sequence further in Chapter 3.

Let $\beta$ be the greatest lower bound of $\phi(P, r)$. We can find $P_{0}$ and $r_{0}$ so that $\phi\left(P_{0}, r_{0}\right)<\beta+\varepsilon$. As indicated above we can show that $\phi$ increases with $r$ and we use this to show that we can find $r_{1}$ such that for every sphere $S(P, r) \subset S\left(P_{0}, r_{1}\right)$ $\beta \leqslant \phi(P, r)<\beta+\varepsilon$. Clearly the surface will be very well behaved in $S\left(P_{0}, r_{1}\right)$. We show first that in each $S(P, r) \subset S\left(P_{0}, r_{1}\right)$ there will be a substantial spherical hole containing no points of $S$. This depends effectively speaking only on $S$ having zero three-dimensional measure. Now we can show that at a point of $S$ on the boundary of this hole there will be a tangent plane in quite a strong sense. Thus we obtain an everywhere dense set of tangential bits of $S$. Next we show that unless most of these tangent bits pass through $P$ the equation $\int l(P, r) \leqslant A(P, r)$ can be strengthened to $\int l(P, r)<(1-k) A(P, r)$ whence we obtain $\int l(P, r)<\frac{1}{2}(1-k) r l(P, r)$ which leads to a contradiction. Thus most of these tangent bits pass through all points $P \in S$ near them and hence they all lie in the same plane. The existence of a tangent plane now gives $\beta \geqslant 1$. Thus $\phi(P, r) \geqslant 1$ whence a direct application of the definition of Hausdorff spherical measure gives lower semi-continuity and hence the existence theorem. We have incidentally proved a tangential property which is then used in Chapter 4 to prove local Euclideanness by means of theorem 3 which may be of in. dependent interest since it does not use the fact that the set is a surface but is applicable to any point set.

This theorem (1) says roughly that if the contents of each sphere $S(P, r)$ with $P \in S$ lie in a narrow strip of width less than $2^{-2000 N^{*}} r$, then the surface is locally Euclidean. It should be noted that the strip is allowed to vary both with $P$ and $r$.

The proof consists of constructing inductively a series of disks which converge to a piece of the surface and are such that each disk is the image of the previous

(1) Stated near the end of Chapter 4. 
one in such a manner that both the mapping function and its inverse are Lipschitz with constant $k$ where $k$ is fixed throughout the series. Once this series has been constructed the rest is easy.

In order to construct this series suppose we consider neighbourhoods of a definite more or less fixed size on the surface; from the point of view of ignoring smaller variations than this there will be a tangential direction at each point of the surface. Suppose we have constructed a disk which is parallel to the surface at each point and also a system of moving co-ordinate axes attached to each point near $S$, which vary subject to a Lipschitz condition and whose first $m$ axes lie in the tangent plane. Consider now a system of much smaller neighbourhoods (whose size must be neither too big nor too small). We can take a network of points $X_{i}$ on $S$ whose distances apart are of the order of magnitude of these neighbourhoods and which "cover" the whole of $S$. At each of these points we set up co-ordinate axes appropriate to the tangential direction given by the new size of neighbourhood. We now refer the directions of these co-ordinate axes to the previous system of "larger scale" moving co-ordinate axes. The direction cosines so referred will not vary very rapidly and we can now perform an averaging process by which we obtain a new system of Lipschitzian moving axes more or less equal to the new smaller scale axes at the $X_{i}$. These new axes will be defined at each point $P$ of the disk already defined and each point $X_{i}$ of our network will have co-ordinates with respect to these axes. For each $P$ we now perform an averaging process of these co-ordinates over the $X_{i}$. This maps $P$ onto a new point $P^{*}$; as $P$ varies on the disk, $P^{*}$ traces out a new disk through the $X_{i}$ and parallel to the surface from the point of view of the new smaller neighbourhoods. This completes the induction.

This process involves several averaging processes, each of which introduces complicated estimations; in addition we must consider a large number of small quantities which must be chosen to have the right relationship in order of magnitude, consequently the proof is in parts unavoidably messy-particularly lemma 4. This completes the proof of the main theorem. To prove theorem 1 we must first prove that when $G$ is the group of integers modulo 2 and $m=2, n=3$ the density does not exceed one at any point. This is done in Chapter 5. Consider the part of the surface in the sphere $S(P, r)$; dilate this to unit radius. In this way we obtain a series of minimum surfaces in the unit sphere. These surfaces will converge in a subsequence to a surface of minimum area whose area is then shown to be equal to that of the cone with the same boundary. Hence we have a cone of minimum area which it can be shown by methods of the calculus of variations must be a plane disc. 
The proof of Theorem 1 is then completed by means of a simple construction, while Theorem 2 is shown to follow almost at once from the main theorem with $G=$ group of reals modulo 1 .

I will now discuss some as yet unsolved problems.

\section{Problem 1 .}

A surface of minimum area is known to be locally Euclidean p.p.; it remains to prove that it is differentiable and that it is a minimal surface in the sense of differential geometry, where $m \geqslant 3$.

\section{Problem 2.}

The structure of surfaces of minimum area at points where they are not locally Euclidean. I conjecture that for each $m, n$ there exists $k=k(m, n)$ such that a minimum surface will consist of the union of at most $k$ half dises at every point and that when $G$ is the group of integers mod 2 the number of half discs is even in every case. The reader should study examples $7,8,9$ of the appendix. We cannot demand that the surface shall be locally a disc everywhere except when $m=2$ and $n=3$ and $G \equiv$ Integers $\bmod 2$. For consider $m=2, n=4$ and a boundary consisting of $x^{2}+y^{2}=1, z=0, t=0$ plus $z^{2}+t^{2}=1, x=0, y=0$. The origin is a singular point. An extension of the method of Chapter 5 might again be helpful. Certainly the crux of the problem seems to be to prove that if a cone is a minimum surface then it consists of plane bits.

\section{Problem 3.}

If $m>2$ it has not been proved that there exists a minimum in the class of discs. Suppose we take all the surfaces $S_{n}$ of Chapters 2 and 3 to be dises it might still be possible to carry out everything to this point-care would be needed with Chapter 1. We then have a situation where a sequence of dises $S_{n}$ whose area approaches the minimum converges to a set $S$ lower semi-continuously. We must then prove that $S$ is a disc: very difficult.

\section{Problem 4.}

A very special case of part of the last problem but possibly the crux. Let $M$ be a manifold with boundary $C$. Let $D_{n}$ be discs with the same boundary. Let $\mu$ be the lower bound of the areas of discs with boundary $C$. Suppose $D_{n} \rightarrow M$ and $\Lambda^{2} D_{n} \rightarrow \Lambda^{2} M=\mu$. Prove that $M$ is a disc. Also the same for $m$-dimensional sets. 


\section{Problem 5.}

If $m=2, n=3, G=$ Integers $\bmod 2$ and the boundary is a finite set of disjoint polygons prove that the surface of minimum area is a manifold. This should not be hard. (1)

\section{Problem 6.}

To find a definition of surface which includes examples 8 and 9 of the appendix. The point appears to be that a physical deformation cannot tear apart points once they have been brought together and hence lies somewhere between a deformation retraction and an isotopy. It should certainly be studied. It is not even obvious how best to define a "physical deformation".

\section{Problem. 7.}

Suppose $D$ is an open set on a surface $S$. Let $\mu(D)$ be the lower bound of the areas of surfaces with the same boundary as $D$. Consider the class of finite sets of disjoint sets $D_{i}$. Define $A(S)$ as the least upper bound of $\sum \mu\left(D_{i}\right) . A(S)$ considered as the area of the surface should be a powerful tool. Perhaps the $D_{i}$ should be taken to be the part of $S$ in a sphere; it seems likely that this will give the same value but might be easier to handle. (This in itself might be interesting to prove.)

The author used $A(S)$ with the $D_{i}$ restricted to be simply connected Jordan domains to investigate two dimensional discs in three space. In this case $A(S)$ was shown to be lower semi-continuous and equal to the Lebesgue area and it was then proved that, if $A(S)<\infty, A(S)$ equalled the Hausdorff measure of the set points of $S$ where a tangent plane exists. These results are likely to extend in some form; the last result would be particularly interesting for $m>2$, or even for $m=2, n>3$.

\section{PROBLEM 8.}

In theorem 1, provided we assume that the curves of $A$ are tamely embedded in three space, it might be possible to replace $\mathcal{G}$ by the class of sets $S$ such that for each $\varepsilon>0$ there exists a manifold with boundary $A$ coinciding with $S$ at points further than $\varepsilon$ from $A$.

It is not clear if this is a more interesting form of the theorem but it is much harder to prove, as the essential restriction on $A$ shows.

\section{Problem 9.}

This is a minor problem concerned with the question of whether the term intrinsic area has any real meaning. Suppose $S_{1}$ and $S_{2}$ are two topological 2 -spheres

(1) This has now been solved by the Author. 
in three-space. Suppose given a homeomorphism $\phi$ between them such that if $C_{1}=\phi\left(C_{2}\right)$ and $C_{1}, C_{2}$ are continua on $S_{1}$ and $S_{2}$ then their diameters are equal. Prove that $S_{1}$ and $S_{2}$ are congruent apart from translation, rotation or reflection. For polyhedra the result is easy.

\section{Problem 10.}

This is to generalise Theorem 2 to the case where the boundary is any manifold. Example 4 of the Appendix shows that the problem is non-trivial.

Conjecture:-If $M \subset S$ is an $(m-1)$-dimensional manifold (and $G=$ reals $\bmod$ one) the necessary and sufficient condition that $S$ is a surface with boundary $M$ is that there shall be no $(m-1)$-dimensional set $M^{*}, S \supset M^{*} \supset M$ which is a retract of $S$.

\section{Notation.}

Throughout the following work we use the following symbols:-

Suppose $P$ is a point, $X$ a set of points, $\Pi$ a plane, $r$ a positive number, $S_{\alpha}$ a surface, then $S(P, r)$ is the closed solid sphere with centre $P$ and radius $r$. $S^{0}(P, r)$ is the open solid sphere. $s(P, r)$ is the surface of the sphere. $(X, r)$ is the set of points whose distance from $X$ does not exceed $r$. $C(P, X)$ is the cone with vertex $P$ and base $X$.

$C\left(\prod, X\right)$ is $\sum_{P \in X} I_{p}$ where $I_{p}$ is the closed interval joining $P$ to its projection on $\Pi$. $K_{\alpha}(P, r)=S_{\alpha} S(P, r)$. $l_{\alpha}(P, r)=S_{\alpha} s(P, r)$.

$\Lambda^{m} X$ is the $m$-dimensional Hausdorff spherical measure as defined at the beginning of Chapter 1.

LEMMAS $1 A-26 A$ belong to the appendix.

Lemmas 1-15 belong to Chapter 1 .

LEMMAS $1^{*}-7^{*}$ belong to Chapter 3 .

LEMMAS $1^{\prime}-5^{\prime}$ belong to Chapter 5 .

Chapter 4 is written independently from the rest of the paper and can be read on its own; its lemmas are numbered 1-9.

The group $G$ involved in the definition of a surface is kept fixed throughout the bulk of the paper and will therefore be suppressed. Moreover I use the following notational convention:- 
Suppose $\left\{A_{i}\right\},\left\{B_{i}\right\}$ are two sets of closed sets and that $X=\sum A_{i}+\sum B_{i}$, and that $L_{i} \subset H_{m}\left(A_{i}\right)$ and $M_{i} \subset H_{m}\left(B_{i}\right)$ while $i_{*}$ denotes the natural inclusion homomorphism then

$$
\sum L_{i}=\sum M_{i}
$$

will mean

$$
\sum i_{*}\left(X, A_{i}\right) L_{i}=\sum i_{*}\left(X, B_{i}\right) M_{i}
$$

This notation is very convenient when such equations are quoted for referenceparticularly if the $A_{i}$ and $B_{i}$ are very complicated expressions.

There is no possibility of ambiguity provided the conventional equation is not manipulated, but the reader must be warned that without reference back to the parent equation any manipulation may lead to false results.

\section{Chapter 1}

Suppose $X$ is a set in $N$-dimensional Euclidean space. Consider a set of spheres $S\left(P_{i}, r_{i}\right)$ such that $r_{i}<\delta$ and $X \subset \sum_{i} S\left(P_{i}, r_{i}\right)$. I define $\Lambda_{\delta}^{m} X$ to be the lower bound of $\sum_{i} W_{m} r_{i}^{m}$ taken over all such sets of spheres; where $W_{m}$ is the elementary volume of an $m$-dimensional solid unit sphere. Let then $\Lambda^{m} X=\lim _{\delta \rightarrow 0} \Lambda_{\delta}^{m} X$.

LEMMA 1. If $\Lambda^{m} X<\infty$, and we have a class of spheres such that there are arbitrarily small spheres of the class with centre at any point of $X$, then we can select a non-overlapping set of spheres of the class containing almost all of $X$.

This may be proved by a straightforward generalisation of the proof of the first main theorem in [2].

Lemma 2. If $X$ is an m-dimensional unit cube then $\Lambda^{m} X=1$. Moreover if $P \rightarrow P^{*}$ is a mapping of the space into itself such that $\left|P^{*} Q^{*}\right| \leqslant \lambda|P Q|$ and $S$ is a set of points such that $S \rightarrow S^{*}$ then $\Lambda^{m} S^{*} \leqslant \lambda^{m} \Lambda^{m} S$.

This result is, of course, classical. It can be deduced immediately from the definition by the use of lemma 1 .

LEMMA 3. If $\Lambda^{m} X<\infty$ then

$$
0<\varlimsup_{r \rightarrow 0} \frac{\Lambda^{m} X S(P, r)}{W_{m} r^{m}} \leqslant 1
$$

at almost all points $P \in X$. Moreover we can find a $G_{\delta}$ set containing $X$ and of the same measure.

This is a straightforward generalisation of results in the early part of [1]. 
Lемма 4. Suppose $X_{h}$ is the set of points of $X$ at a distance $h$ from a fixed $M$-dimensional plane in $N$-dimensional Euclidean space, $N-1 \geqslant M \geqslant 0$. Then

$$
\int_{0}^{\infty} \Lambda^{m-1} X_{h} d h \leqslant \Lambda^{m} X .
$$

This is a straightforward generalisation of lemma 3 of [3].

L ммм 5. If $X$ is an $(m-1)$-dimensional polyhedron then the cone $C(P, X)$ with vertex $P$, on $X$ will be an m-dimensional polyhedron and if $X \subset S(P, r)$, then

$$
\Lambda^{m} C(P, X) \leqslant \frac{r \Lambda^{m-1} X}{m} .
$$

This is clear by lemma 2 .

LEMMA 6. If $\prod$ is an $M$-dimensional plane let $C\left(\prod, X\right)=\sum_{P \in X} I_{P}$ where $I_{P}$ is the interval joining $P$ to its projection on $\Pi$. Then if $X \subset(\Pi, r)$ and $N-1 \geqslant M \geqslant 0$

$$
\Lambda^{m} C\left(\prod, X\right) \leqslant \frac{2^{m} W_{m}}{W_{m-1}} r \Lambda^{m-1} X .
$$

Suppose $\left\{S\left(P_{i}, r_{i}\right)\right\}$ is a set of spheres $r_{i}<\delta$ such that

$$
\begin{aligned}
& \sum_{i} S\left(P_{i}, r_{i}\right) \supset X \text { and } \\
& \sum_{i} W_{m-1} r_{i}^{m-1} \leqslant \Lambda_{\delta}^{m-1} X+\delta .
\end{aligned}
$$

Let $P_{i j}, 0 \leqslant j \leqslant\left(r-r_{i}\right) / r_{i}$ be the point on $I_{P_{i}}$, whose distance from $P_{i}$ is $j r_{i}$. If $P \subset S\left(P_{i}, r_{i}\right)$

$$
I_{P} \subset \sum_{j} S\left(P_{i j}, 2 r_{i}\right) \text { and hence }
$$

$\Lambda_{2 \delta}^{m} C(\Pi, X) \leqslant \sum_{i} \frac{r}{r_{i}} W_{m}\left(2 r_{i}\right)^{m} \leqslant \frac{2^{m} W_{m}}{W_{m-1}} r \sum_{i} W_{m-1} r_{i}^{m-1} \leqslant \frac{2^{m} W_{m}}{W_{m-1}} r\left(\Lambda_{\delta}^{m-1} X+\delta\right)$.

Whence letting $\delta \rightarrow 0$ the result follows.

L Eм м 7. If $A=\sum_{j=1}^{n} A_{j}$ where $A_{j} \subset S\left(P_{0}, r_{j}\right)$ and $\varepsilon>0$ there will exist a surface $X$ with boundary $\left(^{1}\right) \supset H_{m-1}^{*}(A)$ such that $X$ is contained in the convex hull of $A+P_{0}$ and

$$
\Lambda^{m} X \leqslant \sum_{j} \frac{r_{j} \Lambda^{m-1} A_{j}}{m}(1+\varepsilon)
$$

(1) $H_{m}^{*}$ is $H_{m}$ if $m>0$ and Ker $\varepsilon$ if $m=0$. 
and there is an m-dimensional polyhedron $X^{\prime}$ contained in $X$ such that

$$
\Lambda^{m}\left(X-X^{\prime}\right)<\varepsilon
$$

If we define $\Lambda^{0} X$ to be the number of points in $X$ if this is finite and $\infty$ otherwise the lemma will be trivially true for $m=1$ by taking $X=X^{\prime}=C\left(P_{0}, A\right)$. I will now prove the lemma by induction. Suppose then that the lemma is true for $m=m_{0}-1$. Let $r_{0}=\max r_{j}$. Take $\delta>0$. If $\Lambda^{m_{0}-1} A_{j} \neq 0$ we may, by Lemma 3 , choose an open set $G_{j} \supset A_{j}$ such that $\Lambda^{m_{\mathrm{e}}-1} A G_{j} \leqslant(1+\delta) \Lambda^{m_{\mathrm{a}}-1} A_{j}$. Thøn at almost all points $P \in A_{j}$ there will exist arbitrarily small $r<\delta$ such that $S(P, r) \subset G_{j}$ and

$$
\Lambda^{m_{0}-1} A S(P, r) \geqslant(1-\delta) \frac{r \Lambda^{m_{\mathrm{o}}-2} A s(P, r)}{m_{0}-1}
$$

for otherwise there will exist $r^{*}$ such that for all $r<r^{*}$ we have, using Lemma 4 ,

$$
\int_{0}^{r} \Lambda^{m_{0}-2} A s(P, t) d t \leqslant \Lambda^{m_{0}-1} A S(P, r) \leqslant \frac{(1-\delta) r \Lambda^{m_{0}-2} A s(P, r)}{m_{0}-1} .
$$

Writing $\int_{0}^{r} \Lambda^{m_{0}-2} A s(P, t) d t=F(r)$, we obtain

$$
F(r) \leqslant \frac{(1-\delta) r}{m_{0}-1} F^{\prime}(r) \text { for almost all } r . \quad \text { Hence } \frac{F^{\prime}(r)}{F(r)} \geqslant \frac{m_{0}-1}{1-\delta} \cdot \frac{1}{r} .
$$

Integrating this from $r_{2}$ to $r_{1}$ we have

$$
\frac{F^{\prime}\left(r_{1}\right)}{F\left(r_{2}\right)} \geqslant\left(\frac{r_{1}}{r_{2}}\right)^{\left(m_{0}-1\right) /(1-\delta)}, r_{2}<r_{1}<r^{*} .
$$

Letting $r_{2} \rightarrow 0$ we obtain $\quad F(r)=o\left(r^{m_{0}-1}\right)$.

Hence there will exist $r_{n}, 2^{-n-1}<r_{n}<2^{-n}<r^{*}$, such that

$$
\Lambda^{m_{0}-2} A s\left(P, r_{n}\right)=o\left(r_{n}^{m_{0}-2}\right) \text { as } n \rightarrow \infty .
$$

Thus by (2) above $\quad \Lambda^{m_{0}-1} A S\left(P, r_{n}\right)=o\left(r_{n}^{m_{0}-1}\right)$ as $n \rightarrow \infty$.

But $\Lambda^{m_{0}-1} A S(P, r)$ is a monotone increasing function of $r$ and hence

$$
\Lambda^{m_{0}-1} A S(P, r)=o\left(r^{m_{0}-1}\right) \text { as } r \rightarrow 0
$$

which can only happen at a set of points $P$ of $\Lambda^{m_{a}-1}$ measure zero, by Lemma 3 . Thus (1) is established. 
We may therefore by Lemma 1 cover almost all of $A$ by a set of non-overlapping spheres $\left\{S\left(P_{i}, \varrho_{i}\right)\right\}$ such that $\varrho_{i}<\delta, P_{i} \in A$ and

$$
\Lambda^{m_{0}-1} A S\left(P_{i}, \varrho_{i}\right) \geqslant(1-\delta) \frac{\varrho_{i} \Lambda^{m_{0}-2} A s\left(P_{i}, \varrho_{i}\right)}{m_{0}-1} .
$$

Moreover if $P_{i} \in A_{j}$ we may suppose that $S\left(P_{i}, \varrho_{i}\right) \subset G_{j}$.

We will choose a finite subset of these non-overlapping spheres such that

$$
\sum_{i} \Lambda^{m_{0}-1} A S\left(P_{i}, \varrho_{i}\right)>\Lambda^{m_{0}-1} A-\delta
$$

Since $\Lambda^{m_{0}-1} A s\left(P_{i}, \varrho_{i}\right)=0$ for all $i$, we then have

$$
\Lambda^{m_{0}-1} \overline{A-\sum_{i} S\left(P_{i}, \varrho_{i}\right)}<\delta
$$

If we write $D_{0}=\overline{A-\sum_{i} S\left(P_{i}, \varrho_{i}\right)}$ and $D_{i}=A S\left(P_{i}, \varrho_{i}\right)$ we will have $A=D_{0}+\sum_{i} D_{i}$ and $D_{i} \cdot D_{i^{\prime}}=0$ if $i \neq i^{\prime} \neq 0$, and $D_{0} \cdot D_{i}=A s\left(P_{i}, \varrho_{i}\right)$.

Hence if $X_{i}$ is a surface with boundary $\supset H_{m_{0}-2}^{*}\left(A s\left(P_{i}, \varrho_{i}\right)\right)$ then by Lemma $15 \mathrm{~A}$

$$
H_{m_{0}-1}\left(D_{0}+\sum_{i} X_{i}\right)+\sum_{i \geqslant 0} H_{m_{0}-1}\left(D_{i}+X_{i}\right) \supset H_{m_{0}-1}(A)
$$

Now by our inductive hypothesis we may choose $X_{i}$ so that it lies in the convex hull of $P_{i}+A s\left(P_{i}, \varrho_{i}\right)$ and

$$
\Lambda_{m_{\mathrm{o}}-1} X_{i} \leqslant \frac{\varrho_{i} \Lambda^{m_{\mathrm{o}}-2} A s\left(P_{i}, \varrho_{i}\right)}{m_{0}-1}(1+\delta)
$$

while there is an $\left(m_{0}-1\right)$-dimensional polyhedron $X_{i}^{\prime} \subset X_{i}$ such that

$$
\Lambda_{m_{\mathrm{a}-1}}\left(X_{i}-X_{i}^{\prime}\right) \leqslant \frac{\delta \Lambda^{m_{0}-1} A S\left(P_{i}, \varrho_{i}\right)}{\Lambda^{m_{0}-1} A} .
$$

In view of (6) above, Lemma $3 \mathrm{~A}$ and $11 \mathrm{~A}$ imply that

$$
X=C\left(P_{0}, D_{0}+\sum_{i} X_{i}\right)+\sum_{i} C\left(P_{i}, D_{i}+X_{i}\right)
$$

is a surface with boundary $\supset H_{m_{0}-1}(A)$.

Since $P_{i} \in A$ and $X_{i}$ lies in the convex hull of $A, X$ will lie in the convex hull of $P_{0}+A$.

$$
\begin{aligned}
& X^{\prime}=C\left(P_{0}, \sum_{i} X_{i}^{\prime}\right) \text { will be a polyhedron and } \\
& X=X^{\prime}+C\left(P_{0}, D_{0}+\sum_{i} X_{i}-X_{i}^{\prime}\right)+\sum_{i} C\left(P_{i}, D_{i}+X_{i}\right)
\end{aligned}
$$




$$
S\left(P_{0}, r_{0}\right)+\sum_{i} S\left(P_{i}, \varrho_{i}\right) \subset S\left(P_{0}, r_{0}+\delta\right)
$$

and so by Lemma 6 and (4), (5), (7) and (8) above

$$
\begin{aligned}
\Lambda^{m_{\circ}}\left(X-X^{\prime}\right) & \leqslant \frac{2^{m_{0}} W_{m_{0}}}{W_{m_{0}-1}}\left\{\left(r_{0}+\delta\right)(\delta+\delta)+\sum_{i} \varrho_{i}\left(1+\frac{1+\delta}{1-\delta}\right) \Lambda^{m_{0}-1} A S\left(P_{i}, \varrho_{i}\right)\right\} \\
& \leqslant \frac{2^{m_{0}} W_{m_{0}}}{W_{m_{0}-1}}\left\{2 \delta\left(r_{0}+\delta\right)+\frac{2 \delta}{1-\delta} \Lambda^{m_{0}-1} A\right\}
\end{aligned}
$$

which is less than $\varepsilon$ provided $\delta$ is small.

Moreover by Lemma 5 and (4) and (7) above

$$
\begin{aligned}
\Lambda^{m_{0}} X^{\prime} \leqslant \sum_{j=1}^{n} \frac{r_{j}+\delta}{m_{0}} \sum_{P_{i} \in A_{j}} \frac{1+\delta}{1-\delta} \Lambda^{m_{0}-1} A S\left(P_{i}, \varrho_{i}\right) & \leqslant \sum_{j=1}^{n} \frac{r_{j}+\delta}{m_{0}} \cdot \frac{1+\delta}{1-\delta} \Lambda^{m_{0}-1} A G_{j} \\
& \leqslant \sum_{j=1}^{n} \frac{r_{j}+\delta}{m_{0}} \cdot \frac{(1+\delta)^{2}}{1-\delta} \Lambda^{m_{0}-1} A_{j},
\end{aligned}
$$

which, by taking $\delta$ small, establishes the lemma.

Lемм A. There exists $K_{m}^{N}<\infty$ such that if $A$ is a bounded set in $N$-dimensional Euclidean space, and $m \geqslant 2$, then there will exist a surface $X$ with boundary $\supset H_{m-1}(A)$, lying in the convex hull of $A$, such that

$$
\Lambda^{m} X \leqslant K_{m}^{N}\left\{\Lambda^{m-1} A\right\}^{m /(m-1)}
$$

and

$$
X \subset\left(A, K_{m}^{N}\left\{\Lambda^{m-1} A\right\}^{1 /(m-1)}\right) .
$$

Write

$$
\left\{\Lambda^{m-1} A\right\}^{1 /(m-1)}=l
$$

Consider first the case $m=2$. Suppose $\Sigma$ is an $(N-1)$-dimensional plane and that we have some Cartesian co-ordinate system in which the axis of $x$ is orthogonal to $\Sigma$. Let $\sum_{t}$ be the set of planes parallel to $\sum$ and passing through the points with $x$ coordinate $t+2 \lambda l$ where $\lambda$ takes all integer values, positive, negative or zero. By Lemma 4

$$
\int_{0}^{2 t} \Lambda^{0}\left(A \sum_{t}\right) d t \leqslant \Lambda^{1} A=l
$$

and hence there will be $t_{0}$ such that $A \sum_{t_{0}}$ is null. Taking $N$ orthogonal systems of planes we see that $A$ may be divided up into a finite number of closed disjoint subsets $A_{i}$, each contained in a cube of side $2 l$. If $P_{i} \in A_{i}$ then $A_{i} \subset S\left(P_{i}, 2 l \sqrt{N}\right)$ 
so that, by Lemma $3 \mathrm{~A}, X=\sum_{i} C\left(P_{i}, A_{i}\right)$ will be a surface with boundary $\supset H_{m-1}(A)$, and by Lemma 6 ,

$$
\Lambda^{2} X \leqslant 2 \cdot 2 l \sqrt{N} \cdot \Lambda^{1} A=4 \sqrt{N} l^{2}
$$

Moreover $X$ is contained in the convex hull of $A$ and

$$
X \subset(A, 2 l \sqrt{N}) \subset(A, 4 l \sqrt{N})
$$

so that taking $K_{2}^{N}=4 \sqrt{N}$ we have our result.

To consider the general case let $P(m, N, k)$ be the proposition:-there exists ${ }^{k} K_{m}^{N}<\infty$ such that given $k$ orthogonal $(N-1)$-dimensional planes in $N$-dimensional Euclidean space and a set $A$ no point of which lies further than $\left\{\Lambda^{m-1} A\right\}^{1 /(m-1)}$ from any of these planes, and $m \geqslant 2$, then there will exist a surface $X$ with boundary $\supset H_{m-1}(A)$, lying in the convex hull of $A$ and such that

$$
\Lambda^{m} X \leqslant{ }^{k} K_{m}^{N}\left\{\Lambda^{m-1} A\right\}^{m /(m-1)}
$$

and

$$
X \subset\left(A,{ }^{k} K_{m}^{N}\left\{\Lambda^{m-1} A\right\}^{1 /(m-1)}\right) .
$$

We have above proved the proposition $P(2, N, k) k \leqslant N$. Consider now $P(m, N, N)$; $\mathrm{i}^{\mathrm{n}}$ this case $A$ will be contained in a cube of side $2 l$ and we may prove the proposition exactly as above. $P\left(m_{0}, N, 0\right)$ is simply the case $m=m_{0}$ of the lemma. Suppose then that $P\left(m_{0}-1, N, 0\right)$ and $P\left(m_{0}, N, k_{0}\right)$ are established; I will deduce $P\left(m_{0}, N, k_{0}-1\right)$ and hence, since we know $P\left(m_{0}, N, N\right)$ we can conclude by down wards induction on $k$ that $P\left(m_{0}, N, 0\right)$ holds and then the lemma will follow by induction on $m$.

Suppose now that $A$ satisfies the hypotheses of $P\left(m_{0}, N, k_{0}-1\right)$. Let $\sum$ be a plane orthogonal to the $k_{0}-1$ planes of that proposition, and suppose we have some Cartesian co-ordinate system in which the $x$ axis is orthogonal to $\Sigma$. Let $\Sigma_{t}$ be the system of planes parallel to $\Sigma$ and passing through the points with $x$ co-ordinate $\lambda l+t$ where $\lambda$ takes all integer values, positive, negative or zero. By Lemma 4

$$
\int_{0}^{l} \Lambda^{m_{0}-2}\left(A \sum_{t}\right) d t \leqslant \Lambda^{m_{0}-1} A=l^{m_{0}-1} .
$$

Hence there is a $t_{0}$ such that

$$
\Lambda^{m_{0}-2}\left(A \sum_{t_{0}}\right) \leqslant l^{m_{0}-2} .
$$

Let $\left\{\sum_{i}\right\}(i=1,2 \ldots n)$ be the smallest set of consecutive planes of $\sum_{t_{0}}$ containing $A \sum_{t_{0}}$. 
Since $A$ is bounded this will be a finite set of planes. There will, by $P\left(m_{0}-1, N, 0\right)$, exist a surface $X_{i}$ with boundary $\supset H_{m_{0}-2}\left(A \sum_{i}\right)$

$$
\Lambda^{m_{0}-1} X_{i} \leqslant{ }^{0} K_{m_{0}-1}^{N}\left\{\Lambda^{m_{0}-2} A \sum_{i}\right\}^{\left(m_{0}-1\right) /\left(m_{0}-2\right)} \text {. }
$$

Moreover we may suppose that $X_{i}$ lies in the convex hull of $A \sum_{i} \subset A$ and, by (9), that

$$
X_{i} \subset\left(A \sum_{i},{ }^{0} K_{m_{0}-1}^{N} l\right)
$$

Let $A_{i}(i=1,2 \ldots n-1)$ be the part of $A$ between $\Sigma_{i}$ and $\sum_{i+1}$, including the points on these planes. Let $A_{0}$ be the part of $A$ on the opposite side of $\Sigma_{1}$ to $\Sigma_{2}$, again including $A \Sigma_{1}$; similarly define $A_{n}$. In order to simplify the notation I will use the symbols $X_{0}$ and $X_{n+1}$ but they will denote null sets. Then $A=\sum_{i} A_{i}, A_{i} \cdot A_{i^{\prime}}=0$ $\left|i-i^{\prime}\right|>1$, and $A_{i} \cdot A_{i+1}=A \sum_{i+1}$. Hence by Lemma $16 \mathrm{~A}$

$$
\sum_{i=0}^{n} H_{m_{0}-1}\left(X_{i}+A_{i}+X_{i+1}\right) \supset H_{m_{0}-1}(A)
$$

Now $X_{i}+X_{i+1}+A_{i}$ satisfies the hypotheses of $P\left(m_{0}, N, k_{0}\right)$ and hence there will exist a surface $Y_{i}$ with boundary $\supset H_{m_{0}-1}\left(X_{i}+A_{i}+X_{i+1}\right)$ lying in the convex hull of $A$ and such that

$$
\Lambda^{m_{0}} Y_{i} \leqslant{ }^{k_{0}} K_{m_{0}}^{N}\left\{\Lambda^{m_{0}-1}\left(X_{i}+X_{i+1}+A_{i}\right)\right\}^{m_{0} /\left(m_{0}-1\right)},
$$

and

$$
Y_{i} \subset\left(A_{i}+X_{i}+X_{i+1},\left\{\Lambda^{m_{0}-1}\left(X_{i}+X_{i+1}+A_{i}\right)\right\}^{1 /\left(m_{0}-1\right)}\right) .
$$

By (12) and Lemma $11 \mathrm{~A} \sum_{i=0}^{n} Y_{i}$ will be a surface with boundary $\supset H_{m_{0}-1}(A)$. By (9), (10), (11) and (14)

$$
Y_{i} \subset\left(A,{ }^{0} K_{m_{0}-1}^{N} l+\left\{2^{0} K_{m_{0}-1}^{N}+1\right\}^{1 /\left(m_{0}-1\right)} l\right) .
$$

Hence if we take ${ }^{k_{0}-1} K_{m_{0}}^{N} \geqslant{ }^{0} K_{m_{0}-1}^{N}+\left\{2^{0} K_{m_{0}-1}^{N}+1\right\}^{1 /\left(m_{0}-1\right)}$

$$
\sum_{i=0}^{n} Y_{i} \subset\left\{A,{ }^{k_{0}-1} K_{m_{0}}^{N} l\right\}
$$

Finally by (9), (10) and (13)

$$
\begin{aligned}
\Lambda^{m_{0}} \sum_{i=0}^{n} Y_{i} & \leqslant{ }^{k_{0}} K_{m_{0}}^{N} \sum_{i=0}^{n}\left\{\Lambda^{m_{\mathrm{o}}-1} X_{i}+\Lambda^{m_{\mathrm{o}}-1} X_{i+1}+\Lambda^{m_{\mathrm{o}}-1} A_{i}\right\}^{m_{0} /\left(m_{\mathrm{o}}-1\right)} \\
& \leqslant{ }^{k_{0}} K_{m_{\mathrm{o}}}^{N}\left\{\sum_{i=0}^{n} \Lambda^{m_{\mathrm{o}}-1} X_{i}+\Lambda^{m_{\mathrm{o}}-1} X_{i+1}+\Lambda^{m_{0}-1} A_{i}\right\}^{m_{\mathrm{o}} /\left(m_{\mathrm{o}}-1\right)}
\end{aligned}
$$

2-60173032. Acta mathematica. 104. Imprimé le 21 septembre 1960 


$$
\begin{aligned}
& \leqslant{ }^{k_{0}} K_{m_{0}}^{N}\left\{l^{m_{0}-1}+2^{0} K_{m_{0}-1}^{N} \sum_{i=1}^{n}\left\{\Lambda^{m_{0}-2} A \sum_{i}\right\}^{\left(m_{0}-1\right) /\left(m_{0}-2\right)}\right\}^{m_{0} /\left(m_{0}-1\right)} \\
& \leqslant{ }^{k_{0}} K_{m_{0}}^{N}\left\{l^{m_{0}-1}+2{ }^{0} K_{m_{0}-1}^{N}\left\{\sum_{i=1}^{n} \Lambda^{m_{0}-2} A \sum_{i}\right\}^{\left(m_{0}-1\right) /\left(m_{0}-2\right)}\right\}^{m_{0} /\left(m_{0}-1\right)} \\
& \leqslant{ }^{k_{0}} K_{m_{\mathrm{o}}}^{N}\left\{l^{m_{\mathrm{o}}-1}+2{ }^{0} K_{m_{0}-1}^{N} l^{m_{\mathrm{a}}-1}\right\}^{m_{\mathrm{o}} /\left(m_{\mathrm{o}}-1\right)} \\
& \leqslant{ }^{k_{0}} K_{m_{0}}^{N}\left\{1+2{ }^{0} K_{m_{0}-1}^{N}\right\}^{m_{0} /\left(m_{0}-1\right)} l^{m_{\mathrm{o}}}
\end{aligned}
$$

which, for a suitable choice of ${ }^{k_{0}-1} K_{m_{0}}^{N}$ is less than ${ }^{k_{0}-1} K_{m_{0}}^{N}\left\{\Lambda^{m_{0}-1} A\right\}^{m_{0} /\left(m_{0}-1\right)}$ so that the lemma is established.

LEMM 9. There exists $1<{ }^{*} K_{m}^{N}<\infty$ such that if $A$ is a set lying on $s(P, r)$ and $\left\{\Lambda^{m-1} A\right\}^{1 /(m-1)}<\frac{r}{{ }^{*} K_{m}^{N}}$, and $m \geqslant 2$, then there will exist a surface $X^{*} \subset s(P, r)$ having boundary $\supset H_{m-1}(A)$ such that

$$
\Lambda^{m} X^{*} \leqslant{ }^{*} K_{m}^{N}\left\{\Lambda^{m-1} A\right\}^{m /(m-1)} .
$$

If $X$ is the surface of Lemma 8 and we take ${ }^{*} K_{m}^{N}>2 K_{m}^{N}$ then

$$
X \subset S(P, r)-S\left(P, \frac{1}{2} r\right)
$$

We may therefore, by Lemma $6 \mathrm{~A}$, take $X^{*}$ to be the conical projection of $X$ onto $s(P, r)$ from $P$.

LEMMA 10. If $C$ is an open cube of side $\alpha$ lying in d-dimensional Euclidean space, $m<d \leqslant N$, and $A$ is a set lying on the surface $c$ of $C$, and $S \subset C$ is a surface with boundary $\supset L_{m-1} \subset H_{m-1}(A)$ such that

$$
\Lambda^{m} S C<\frac{\alpha^{m}}{4^{m}\left\{{ }^{*} K_{m}^{N}\right\}^{m-1}},
$$

then there will exist a surface $S^{*} \subset c$ with boundary $\supset L_{m-1}$ and such that

$$
\Lambda^{m}\left(S^{*}-S\right) \leqslant 2^{m+1} N^{m} \Lambda^{m}(S C) .
$$

Let $P$ be the centre of $C$. By Lemma 4

$$
\int_{1 \alpha \alpha}^{1 / \alpha} \Lambda^{m-1} S s(P, r) d r \leqslant \Lambda^{m}(S C) .
$$

Hence there exists $\frac{1}{4} \alpha<r_{0}<\frac{1}{2} \alpha$ such that

$$
\Lambda^{m-1} S s\left(P, r_{0}\right) \leqslant \frac{4 \Lambda^{m}(S C)}{\alpha} .
$$


Hence $\quad\left\{\Lambda^{m-1} S s\left(P, r_{0}\right)^{1 /(m-1)} \leqslant\left\{\frac{4 \Lambda^{m}(S C)}{\alpha}\right\}^{1 /(m-1)}<\frac{\alpha}{4^{*} K_{m}^{N}}<\frac{r_{0}}{{ }^{*} K_{m}^{N}}\right.$.

Moreover by Lemma $12 \mathrm{~A}$ there exists

$$
g_{m-1}^{1} \subset H_{m-1}\left(S s\left(P, r_{0}\right)\right) \text { and } g_{m-1}^{2} \subset H_{m-1}\left(S s\left(P, r_{0}\right)+A\right)
$$

such that $\overline{S-S\left(P, r_{0}\right)}$ is a surface with boundary $\supset g_{m-1}^{2}$ and

$$
g_{m-1}^{1}+g_{m-1}^{2} \supset L_{m-1} \text {. }
$$

We may thus apply Lemma 9 , so that there exists a surface $X^{*} \subset s\left(P, r_{0}\right)$ with boundary $\supset g_{m-1}^{1}$, such that

$$
\Lambda^{m} X^{*} \leqslant{ }^{*} K_{m}^{N}\left\{\Lambda^{m-1} S s\left(P, r_{0}\right)\right\}^{m /(m-1)} .
$$

By Lemma $11 \mathrm{~A}$ and (16) above $S^{\prime}=X^{*}+\overline{S-S\left(P, r_{0}\right)}$ will be a surface with boundary $\supset L_{m-1}$, and by (15) and (17)

$$
\begin{aligned}
\Lambda^{m}\left(S^{\prime} C\right) & \leqslant \Lambda^{m}(S C)+{ }^{*} K_{m}^{N}\left\{\frac{4 \Lambda^{m}(S C)}{\alpha}\right\}^{m /(m-1)} \\
& \leqslant \Lambda^{m}(S C)+{ }^{*} K_{m}^{N}\left\{\overline{\left(\Lambda^{m} S C\right)^{1 / m}\left({ }^{*} K_{m}^{N}\right)^{(m-1) / m}}\right\}^{m /(m-1)} \\
& \leqslant 2 \Lambda^{m}(S C) .
\end{aligned}
$$

Let now $S^{*}$ be the conical projection of $S^{\prime}$ from $P$ onto c. By Lemma 6 A $S^{*}$ will be a surface with boundary $\supset L_{m-1}$, and since $S^{\prime} c=S c$ and $S^{\prime}$ has no points in $S\left(P, \frac{1}{4} \alpha\right)$ it follows from Lemma 2 that

$$
\Lambda^{m}\left(S^{*}-S\right) \leqslant 2^{m} N^{m} \Lambda^{m}\left(S^{\prime} C\right) \leqslant 2^{m+1} N^{m} \Lambda^{m}(S C) ;
$$

which proves the lemma.

Lемма 11. There exists $K=K(N, m)<\infty$ such that if we have a system of parallel equal open cubes of side a whose centres form a lattice parallet to the cubes and of modulus, $\frac{1}{3} a$, and we have a surface $S$, with boundary $L_{m-1} \subset H_{m-1}(A)$, such that

$$
\Lambda^{m}(S-A)<\frac{\left(2^{m+2} N^{m}\right)^{-N+m} a^{m}}{3^{m} \cdot 4^{m} \cdot\left\{{ }^{*} K_{m}^{N}\right\}^{m-1}}
$$

and $S \subset \overline{C_{0}^{N}}, A \subset c_{0}^{N}$ where $C_{0}^{N}$ is a cube of the system and $c_{0}^{N}$ is its boundary, then if $\left\{C_{i}^{N}\right\}$ is the set of cubes of the system meeting $C_{0}^{N}$ there will exist a surface $S^{*} \subset c_{0}^{N}$ with boundary $\supset L_{m-1}$ such that 


$$
A C_{i}^{N}=0 \Rightarrow S^{*} C_{i}^{N}=0
$$

and

$$
\Lambda^{m}\left(S^{*}-A\right) \leqslant K \Lambda^{m}(S-A)
$$

By Lemma 10 we may assume without loss of generality that $S \subset c_{0}^{N}$ and

$$
\Lambda^{m}(S-A)<\frac{\left(2^{m+2} N^{m}\right)^{-N+m+1} a^{m}}{3^{m} \cdot 4^{m} \cdot\left\{{ }^{*} K_{m}^{N}\right\}^{m-1}}
$$

Now our system of cubes will divide the whole space into non-overlapping cubes of side $\frac{1}{3} a$, each of which is either contained in a cube $C_{i}^{N}$ or does not meet it. Thus we have $N$ sets of non-overlapping open $k$-dimensional cubes, $\left\{D_{j}^{k}\right\}$, of side $\frac{1}{3} a$ such that:-

Each $D_{j}^{k_{0^{-}}-1}$ will be a face of some $D_{j^{\prime}}^{k_{0}}, N \geqslant k_{0} \geqslant 1$.

$$
\begin{gathered}
D_{j}^{k} \cdot C_{i}^{N} \neq 0 \Rightarrow D_{j}^{k} \subset C_{i}^{N}, \quad N-1 \geqslant k \geqslant 0 . \\
c_{0}^{N} \cdot C_{i}^{N} \subset \sum_{k=0}^{N-1} \sum_{D_{j}^{k} \subset C_{i}^{N}} D_{j}^{k} .
\end{gathered}
$$

Let $\Gamma_{k}, k \geqslant 0$, be the class of those $D_{j}^{k}$ such that

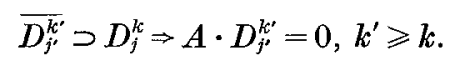

Write $S=S^{N-1}$. Suppose now that we have a surface $S^{t}$ with boundary $\supset L_{m-1}$ lying on $c_{0}^{N}$ and not meeting any $D_{j}^{k} \in \Gamma_{k}, k>t \geqslant 0$ and that

$$
\Lambda^{m}\left(S^{t}-A\right)<\frac{\left(2^{m+2} N^{m}\right)^{m-t} a^{m}}{3^{m} \cdot 4^{m} \cdot\left\{{ }^{*} K_{m}^{N}\right\}^{m-1}} .
$$

If $D_{j}^{t} \in \Gamma_{t}$ then for $k>t$

$$
\overline{D_{j^{\prime}}^{k}} \supset D_{j}^{t} \Rightarrow D_{j^{\prime}}^{k} \in \Gamma_{k} \Rightarrow D_{j^{\prime}}^{k} \cdot S^{t}=0
$$

Hence we can find a set $G_{j}^{t} \supset D_{j}^{t}$, open in $N$-dimensional space, such that

$$
S^{t} G_{j}^{t} \subset D_{j}^{t}
$$

Now by Lemma $12 \mathrm{~A}$ there exist

and

$$
\begin{gathered}
L_{m-1}^{j t} \subset H_{m-1}\left(S^{t}\left\{\overline{D_{j}^{t}}-D_{j}^{t}\right\}\right) \\
\left.L_{m-1}^{t} \subset H_{m-1}\left(A+\sum_{\Gamma_{t}} S^{t} \overline{\left\{D_{j}^{t}\right.}-D_{j}^{t}\right\}\right)
\end{gathered}
$$


such that $S^{t} \cdot \overline{D_{j}^{t}}$ is a surface with boundary $\supset L_{m-1}^{j t}$ and $S^{t}-\sum_{\Gamma_{t}} D_{j}^{t}=S^{t}-\sum_{\Gamma_{t}} G_{j}^{t}$ is a surface with boundary $\supset L_{m-1}^{t}$, while

$$
L_{m-1}^{t}+\sum_{j} L_{m-1}^{j t} \supset L_{m-1}
$$

Consider first the case $t>m$.

In virtue of (23) we can apply Lemma 10 so that there exists a surface $S_{j}^{t_{*}}$ $\subset \overline{D_{j}^{t}}-D_{j}^{t}$, with boundary $L_{m-1}^{j t}$, such that

$$
\Lambda^{m}\left(S_{j}^{t *}-S^{t} \cdot \overline{D_{j}^{t}}\right) \leqslant 2^{m+1} N^{m} \Lambda^{m}\left(S^{t} \cdot D_{j}^{t}\right)
$$

Hence by Lemma $11 \mathrm{~A}$ and (24) $S^{t-1}=\left\{S^{t}-\sum_{\Gamma_{t}} D_{j}^{t}\right\}+\sum_{\Gamma_{t}} S_{j}^{t *}$ is a surface with boundary $\supset L_{m-1}$, and by (25)

$$
\Lambda^{m}\left(S^{t-1}-A\right) \leqslant \Lambda^{m}\left(S^{t}-A\right)+2^{m+1} N^{m} \Lambda^{m}\left(S^{t}-A\right) \leqslant 2^{m+2} N^{m} \Lambda^{m}\left(S^{t}-A\right),
$$

whence in particular

$$
\Lambda^{m}\left(S^{t-1}-A\right) \leqslant \frac{\left(2^{m+2} N^{m}\right)^{m-t+1} a^{m}}{3^{m} \cdot 4^{m} \cdot\left\{{ }^{*} \bar{K}_{m}^{N}\right\}^{m-1}} .
$$

Moreover if $D_{j}^{k} \in \Gamma_{k}, k>t$

$$
S^{t-1} D_{j}^{k}=\left(S^{t}-\sum_{\Gamma_{t}} D_{j^{\prime}}^{t}\right) D_{j}^{k}+\sum_{\Gamma_{t}} S_{j^{*}}^{t *} \cdot D_{j}^{k} \subset S^{t} \cdot D_{j}^{k}+\sum_{\Gamma_{t}} \overline{D_{j^{\prime}}^{t}} \cdot D_{j}^{k}=0
$$

and if $D_{j}^{t} \in \Gamma_{t}$

$$
S^{t-1} D_{j}^{t}=\left(S^{t}-\sum_{\Gamma_{t}} D_{j^{\prime}}^{t}\right) D_{j}^{t}+\sum_{\Gamma_{t}} S_{j^{*}}^{t *} \cdot D_{j}^{t} \subset \sum_{\Gamma_{t}}\left(\overline{D_{j^{\prime}}^{t}}-D_{j^{\prime}}^{t}\right) D_{j}^{t}=0
$$

Thus we obtain a sequence of surfaces $S^{t}$ ending in $S^{m}$ such that for $k>m$

$$
D_{j}^{k} \in \Gamma_{k} \Rightarrow S^{m} \cdot D_{j}^{k}=0
$$

and

$$
\Lambda^{m}\left(S^{m}-A\right) \leqslant\left(2^{m+2} N^{m}\right)^{N-m} \Lambda^{m}(B-A)
$$

On the other hand if $t=m$ and $D_{j}^{m} \cdot A=0, \Lambda^{m} \overline{D_{j}^{m}} S^{m} \leqslant \Lambda^{m}\left(S^{m}-A\right)<\left(\frac{1}{3} a\right)^{m}$, so that by Lemma $8 \mathrm{~A}, L_{m-1}^{j m}=0$, while if $t<m \Lambda^{m-1}\left(\bar{D}_{j}^{t}-D_{j}^{t}\right)=0$ so that $L_{m-1}^{j t}=0$ by Lemma $17 \mathrm{~A}$.

Hence in either case by (24) and Lemmas $11 \mathrm{~A}$ and $1 \mathrm{~A}$

$$
\left.S^{t-1}=\left(S^{t}-\sum_{\Gamma_{t}} D_{j}^{t}\right)+\sum_{\Gamma_{t}} \overline{\left(D_{j}^{t}\right.}-D_{j}^{t}\right) S^{t}=S^{t}-\sum_{\Gamma_{t}} D_{j}^{t}
$$

is a surface with boundary $\supset L_{m-1}$.

We can thus construct a surface $S^{-1}=S^{*}$ which does not meet any $D_{j}^{k} \in \Gamma_{k}$, $k \geqslant 0$, and by $(27)$ 


$$
\Lambda^{m}\left(S^{*}-A\right) \leqslant\left(2^{m+2} N^{m}\right)^{N-m} \Lambda^{m}(S-A) .
$$

On the other hand if $C_{i}^{N} \cdot A=0$ we have for any $D_{j}^{k} \subset C_{i}^{N}$ by (19)

$$
\overline{D_{j^{\prime}}^{k^{\prime}}} \supset D_{j}^{k} \Rightarrow \overline{D_{j^{\prime}}^{k^{\prime}}} \cdot C_{i}^{N} \neq 0 \Rightarrow D_{j^{\prime}}^{k^{\prime}} \cdot C_{i}^{N} \neq 0 \Rightarrow D_{j^{\prime}}^{k^{\prime}} \subset C_{i}^{N} \Rightarrow D_{j^{\prime}}^{k^{\prime}} \cdot A=\mathbf{0}
$$

and hence $D_{j}^{k} \subset C_{i}^{N} \Rightarrow D_{i}^{k} \in \Gamma_{k}$.

Thus by (20) $S^{*} \cdot C_{i}^{N}=0$ which establishes the lemma.

Lемм а 12. If $\sum$ is an m-plane through $P, \varepsilon<\frac{1}{2}, A \subset s(P, r)\left(\sum, \varepsilon r\right), L_{m-1} \subset H_{m-1}(A)$ and $X$ is a surface with boundary $\supset L_{m-1}$ then either

$$
\Lambda^{m} X \geqslant W_{m} r^{m}-\Lambda^{m-1} A \cdot \frac{2^{2 m} W_{m}}{W_{m-1}} \varepsilon r
$$

and the projection of $X$ onto $\sum$ contains $\left.\sum S(P, r)(1-\varepsilon)\right)$ or there exists a surface $X^{\prime}$ with boundary $\supset L_{m-1}$ such that

$$
\Lambda^{m} X^{\prime} \leqslant \Lambda^{m-1} A \cdot \frac{2^{2 m} W_{m}}{W_{m-1}} \varepsilon r .
$$

Let $A^{*}$ be the conical projection from $P$ onto $s(P, r)$ of the orthogonal projection of $A$ onto $\sum$, and let $C$ be the conical projection from $P$ onto $s(P, r)$ of $C(\Sigma, A)$. By Lemma $10 \mathrm{~A}$ if $K$ is the algebraic boundary of $C$ in $A+A^{*}$ then there will exist $L_{m-1}^{*} \subset H_{m-1}\left(A^{*}\right)$ such that

and

$$
K+L_{m-1}^{*} \supset L_{m-1}
$$

Now by Lemmas 6 and 2

$$
\Lambda^{m} C \leqslant \frac{2^{m} W_{m}}{W_{m-1}} \varepsilon r \cdot \Lambda^{m-1} A \cdot\left|\frac{r}{(1-\varepsilon) r}\right|^{m} \leqslant \frac{2^{2 m} W_{m}}{W_{m-1}} \cdot \varepsilon r \cdot \Lambda^{m-1}(A) .
$$

Now if $L_{m-1}^{*}=0$ we may by Lemmas $11 \mathrm{~A}$ and $1 \mathrm{~A}$ take $X^{\prime}=C+A^{*}=C$ and if $L_{m-1}^{*} \neq 0$ then by Lemmas $11 \mathrm{~A}, 6 \mathrm{~A}$ and $8 \mathrm{~A}$ the projection of $X+C$ onto $\sum$ will contain $\sum S(P, r)$ from which the lemma follows.

LEMMA 13. If $S$ is a surface with boundary $\supset L_{m-1} \subset L_{m-1}(A)$ and $G$ is an open set such that $A \cdot \bar{G}=0$ then there will exist $L_{m-1}^{*} \subset H_{m-1}(S(\bar{G}-G))$ such that $S \dot{G}$ is a surface with boundary $\supset L_{m-1}^{*}$ and if $X$ is a surface with boundary $\supset L_{m-1}^{*}$ then $(S-G)+X$ will be a surface with boundary $\supset L_{m-1}$.

This follows at once from Lemmas $11 \mathrm{~A}$ and $12 \mathrm{~A}$. 
LEMma 14. The class of surfaces with boundary $\supset L$ is locally sequentially compact.

By $[7, \S 28]$, if $S_{n}$ is any sequence of surfaces with boundary $\supset L$ there will exist a subsequence $S_{n_{i}}$ converging to a set $S$ in the sense that for any $\varepsilon>0 S_{n_{i}} \subset(S, \varepsilon)$ and $S \subset\left(S_{n_{i}}, \varepsilon\right)$ for large $n_{i}$.

Moreover

$$
S=\prod_{k=1}^{\infty} \overline{\sum_{i \geqslant k} S_{n_{i}}}
$$

and hence by Lemmas $7 \mathrm{~A}$ and $21 \mathrm{~A}, S$ is a surface with boundary $\supset L$.

LEMMA 15. If $S$ is a surface with boundary $\supset L$ then there will exist a proper surface $S^{0} \subset S$ also with boundary $\supset L$.

This follows from Lemma $21 \mathrm{~A}$.

\section{Chapter 2}

Consider a system $T_{n}$ of equal parallel open cubes whose sides have length $2^{-2 n}$ and whose centres form an $N$-dimensional lattice parallel to the sides of the cubes and of modulus $2^{-2 n} / 3$. Let

$$
\begin{aligned}
& r_{n}=2^{-n} \\
& \sigma_{n}=\left(3 r_{n} 2^{2 n}\right)^{N} \\
& u_{n}=(2 K)^{-\sigma_{n}} \\
& \varepsilon_{n}=\min \left\{\frac{1}{2}\left(\frac{r_{n}}{8 m}\right)^{m}\left(\frac{u_{n}}{K_{m}^{N}}\right)^{m-1} ; \frac{\left(2^{m+2} N^{m}\right)^{-N+m+1} \cdot 2^{-2 n m}}{(2 K)^{\sigma_{n}} \cdot u_{n} \cdot 3^{m} \cdot 4^{m} \cdot\left\{{ }^{*} K_{m}^{N}\right\}^{m-1}}\right\},
\end{aligned}
$$

where $K>1, K_{m}^{N}$ and ${ }^{*} K_{m}^{N}$ are the constants defined in Lemmas 11,8 and 9 respectively.

Suppose now that $\Gamma$ is a closed set and that $L_{m-1} \subset H_{m-1}(\Gamma)$. Let $\mu\left(L_{m-1}\right)$ be the greatest lower bound of $\Lambda^{m} S$ taken over all sets $S$ which are surfaces with boundary $\supset L_{m-1}$. Let $\alpha_{n}(S)$ be the number of cubes of $T_{n}$ having points belonging to $S$. Let $\alpha_{n}$ be the greatest lower bound of $\alpha_{n}(S)$ taken over all surfaces $S$ with boundary $\supset L_{m-1}$ and such that

$$
\Lambda^{m} S<\mu\left(L_{m-1}\right)+\varepsilon_{n} .
$$

Since the $\alpha_{n}(S)$ are positive integers $\alpha_{n}$ will be attained and so we have a surface $S_{n}$ with boundary $\supset L_{m-1}$ meeting only $\alpha_{n}$ cubes of $T_{n}$ and such that

$$
\Lambda^{m} S_{n}<\mu\left(L_{m-1}\right)+\varepsilon_{\tilde{n}} \text {. }
$$

Let $\Gamma_{n}$ be the class of spheres not meeting $\Gamma$ and with centre on $S_{n}$. 
Suppose that there exists a sphere $S(P, r) \in \Gamma_{n}$ such that

$$
r>\frac{1}{2} r_{n} \text { and } \Lambda^{m} K_{n}(P, r)<2 \varepsilon_{n} .
$$

Then we will show that there exists $r^{\prime}, 3 r_{n} / 8>r^{\prime}>r_{n} / 4$ such that

$$
K_{m}^{N}\left\{\Lambda^{m-1} l_{n}\left(P, r^{\prime}\right)\right\}^{m /(m-1)} \leqslant u_{n} \int_{0}^{r^{\prime}} \Lambda^{m-1} l_{n}(P, t) d t
$$

For if not

$$
\begin{gathered}
\left\{\frac{K_{m}^{N}}{u_{n}}\right\}^{(m-1) / m} \frac{\frac{d}{d x} F_{n}(P, x)}{\left\{\boldsymbol{F}_{n}(P, x)\right\}^{(m-1) / m}}>1 \text { for almost all } x, \frac{3}{8} r_{n}>x>\frac{1}{4} r_{n} \\
\left(F_{n}(P, r)=\int_{0}^{r} \Lambda^{m-1} l_{n}(P, t) d t\right) .
\end{gathered}
$$

Integrating this from $r_{n} / 4$ to $3 r_{n} / 8$,

$$
m\left\{\frac{K_{m}^{N}}{u_{n}}\right\}^{(m-1) / m}\left[\left\{F_{n}\left(P, \frac{3}{8} r_{n}\right)\right\}^{1 / m}-\left\{F_{n}\left(P, \frac{1}{4} r_{n}\right)\right\}^{1 / m}\right]>\frac{1}{8} r_{n}
$$

so that by Lemma 4 and the definition of $\varepsilon_{n}$,

$$
\Lambda^{m} K_{n}(P, r) \geqslant F_{n}\left(P, \frac{3}{3} r_{n}\right)>\left(\frac{r_{n}}{8 m}\right)^{m}\left(\frac{u_{n}}{K_{m}^{N}}\right)^{m-1} \geqslant 2 \varepsilon_{n} .
$$

a contradiction with (2) above which establishes (3).

By Lemmas 8, 13 and 4 there will therefore exist a set $K_{n}^{*}\left(P, r^{\prime}\right)$ such that

$$
\begin{gathered}
S_{n}^{*}=\left\{S_{n}-K_{n}\left(P, r^{\prime}\right)\right\}+K_{n}^{*}\left(P, r^{\prime}\right) \text { is a surface with boundary } \supset L_{m-1}, \\
S_{n}^{*}-K_{n}^{*}\left(P, r^{\prime}\right)=S_{n}-K_{n}\left(P, r^{\prime}\right), \\
S_{n}^{*} S\left(P, r^{\prime}\right)=K_{n}^{*}\left(P, r^{\prime}\right), \\
\Lambda^{m} K_{n}^{*}\left(P, r^{\prime}\right) \leqslant u_{n} \int_{0}^{r^{\prime}} \Lambda^{m-1} l_{n}(P, t) d t \leqslant u_{n} \Lambda^{m} K_{n}\left(P, r^{\prime}\right) .
\end{gathered}
$$

and

Let $T_{n}(P)$ be the class of cubes of $T_{n}$ contained in $S\left(P, \frac{1}{2} r_{n}\right) . T_{n}(P)$ will have at most $\left(3 r_{n} 2^{2 n}\right)^{N}=\sigma_{n}$ members, let them be $C_{1}, C_{2} \ldots C_{\sigma_{n}}$.

I will now inductively define at sequence of at most $\sigma_{n}+1$ surfaces $S_{n}^{i}$ as follows:-

Let $S_{n}^{0}=S_{n}^{*}$. Suppose that we have defined a surface $S_{n}^{i_{0}-1}$ with boundary $\supset L_{m-1}$ and that 


$$
\begin{gathered}
S_{n}^{i_{0}-1} \supset S_{n}-K_{n}\left(P, r^{\prime}\right), \\
\Lambda^{m}\left\{S_{n}^{i_{0}-1}-\left(S_{n}-K_{n}\left(P, r^{\prime}\right)\right)\right\} \leqslant(2 K)^{i_{\mathbf{a}}-1} \Lambda^{m} K_{n}^{*}\left(P, r^{\prime}\right)
\end{gathered}
$$

and if $i \leqslant i_{0}-1, i_{0}>1$ then

$$
\left[S_{n}-K_{n}\left(P, r^{\prime}\right)\right] C_{i}=0 \Rightarrow S_{n}^{i_{0}-1} C_{i}=0
$$

while for $C \in T_{n}, i_{0}>1$

$$
S_{n}^{i_{a}-2} C=0 \Rightarrow S_{n}^{i_{0}-1} C=0
$$

If now $\left[S_{n}-K_{n}\left(P, r^{\prime}\right)\right] C_{i_{0}} \neq 0$ or if $S_{n}^{i_{0}-1} C_{i_{0}}=0$, I define $S_{n}^{i_{0}}$ by

otherwise we have

$$
S_{n}^{i_{0}}=S_{n}^{i_{a}-1}
$$

$$
\left[S_{n}-K_{n}\left(P, r^{\prime}\right)\right] C_{i_{0}}=\mathbf{0}
$$

and hence by (8)

$$
\begin{aligned}
\Lambda^{m} S_{n}^{i_{0}-1} C_{i_{0}} & \leqslant \Lambda^{m}\left[S_{n}^{i_{0}-1}-\left(S_{n}-K_{n}\left(P, r^{\prime}\right)\right)\right] C_{i_{0}}+\Lambda^{m}\left[S_{n}-K_{n}\left(P, r^{\prime}\right)\right] C_{i_{0}} \\
& \leqslant(2 K)^{i_{0}-1} \Lambda^{m} K_{n}^{*}\left(P, r^{\prime}\right) .
\end{aligned}
$$

Now by (6), (2) and the definition of $\varepsilon_{n}$

$(2 K)^{i_{0}-1} \Lambda^{m} K_{n}^{*}\left(P, r^{\prime}\right) \leqslant(2 K)^{\sigma_{n}-1} u_{n} \Lambda^{m} K_{n}\left(P, r^{\prime}\right) \leqslant(2 K)^{\sigma_{n}} u_{n} \varepsilon_{n} \leqslant \frac{\left(2^{m+2} N^{m}\right)^{-N+m+1} \cdot 2^{-2 n m}}{3^{m} \cdot 4^{m} \cdot\left\{K_{m}^{*}\right\}^{m-1}}$

and we may apply Lemmas 11 and 13 to construct a set $S_{n}^{* i_{0}-1}$ such that if

$$
S_{n}^{i_{0}}=\left(S_{n}^{i_{0}-1}-C_{i_{0}}\right)+S_{n}^{* i_{0}-1}
$$

then $S_{n}^{i_{0}}$ is a surface with boundary $\supset L_{m-1}$ and

$$
\begin{gathered}
\Lambda^{m}\left(S_{n}^{* i_{0}-1}-S_{n}^{i_{0}-1}\right) \leqslant K \Lambda^{m}\left(S_{n}^{i_{0}-1} C_{i_{0}}\right), \\
S_{n}^{* i_{0}-1} \subset \bar{C}_{i_{0}}-C_{i_{0}}
\end{gathered}
$$

and for any $C \in T_{n}$

$$
S_{n}^{i_{0}-1} \bar{C}_{i_{0}} C=0 \Rightarrow S_{n}^{* i_{0}-1} C=0
$$

Let $\left(7^{\prime}\right),\left(8^{\prime}\right),\left(9^{\prime}\right)$ and $\left(10^{\prime}\right)$ be equations $(7),(8),(9)$ and $(10)$ with $i_{0}$ substituted for $i_{0}-1$. $\left(7^{\prime}\right)$ follows from (7), (12) and (14); (8') follows from (14), (8), (15) and (13). (9') follows from (9), (14), (16) and (17); (10') follows from (14) and (17). Thus the inductive definition of the $S_{n}^{i}$ is established. 
Now if $n>N$ and $C \in T_{n}$

$$
C S\left(P, r^{\prime}\right) \neq 0 \Rightarrow C S\left(P, \frac{3}{8} r_{n}\right) \neq 0 \Rightarrow C \subset S\left(P, \frac{1}{2} r_{n}\right) \Rightarrow C \in T_{n}(P)
$$

Thus if $S_{n} C=0$ either $C S\left(P, r^{\prime}\right) \neq 0$ so that $C \in T_{n}(P)$ and $S_{n}^{\sigma_{n}} C=0$ by $(9)$; or else $C S\left(P, r^{\prime}\right)=0$ so that

$$
S_{n}^{0} C=S_{n}^{*} C \subset S_{n} C+K_{n}^{*}\left(P, r^{\prime}\right) C=0
$$

whence $S_{n}^{\sigma_{n}} C=0$ by $(10)$.

On the other hand there will be a cube $C$ of $T_{n}$ containing $P$ and then, since $n>N, C \subset S\left(P, r_{n} / 4\right) \subset S\left(P, r^{\prime}\right)$ so that by (18) and (9) $S_{n}^{\sigma_{n}} C=0$. Thus $S_{n}^{\sigma_{n}}$ will have points in fewer cubes of $T_{n}$ than $S_{n}$. But by (8), (6) and the definition of $u_{n}$

$$
\begin{aligned}
\Lambda^{m} S_{n}^{\sigma_{n}} & \leqslant(2 K)^{\sigma_{n}} \Lambda^{m} K_{n}^{*}\left(P, r^{\prime}\right)+\Lambda^{m}\left(S_{n}-K_{n}\left(P, r^{\prime}\right)\right) \\
& \leqslant(2 K)^{\sigma_{n}} u_{n} \Lambda^{m} K_{n}\left(P, r^{\prime}\right)+\Lambda^{m}\left(S_{n}-K_{n}\left(P, r^{\prime}\right)\right) \leqslant \Lambda^{m} S_{n} .
\end{aligned}
$$

This contradicts the definition of $\alpha_{n}$, and hence (2) above must be false.

Thus if $r>\frac{1}{2} r_{n}$ and $S(P, r) \in \Gamma_{n}$

$$
\Lambda^{m} K_{n}(P, r) \geqslant 2 \varepsilon_{n}
$$

Thus by Lemmas 8 and 13

$$
K_{m}^{N}\left\{\Lambda^{m-1} l_{n}(P, r)\right\}^{m /(m-1)} \geqslant \Lambda^{m} K_{n}(P, r)-\varepsilon_{n} \geqslant \frac{1}{2} \Lambda^{m} K_{n}(P, r) \geqslant \frac{1}{2} \int_{0}^{r} \Lambda^{m-1} l_{n}(P, t) d t .
$$

That is

$$
\frac{\frac{d}{d x} F_{n}(P, r)}{\left\{F_{n}(P, r\}^{(m-1) / m}\right.} \geqslant\left(2 K_{m}^{N}\right)^{-(m-1) / m}
$$

Integrating we find that

$$
\frac{1}{m}\left\{F_{n}(P, r)\right\}^{1 / m}-\frac{1}{m}\left\{F_{n}\left(P, \frac{1}{2} r_{n}\right)\right\}^{1 / m} \geqslant\left(r-\frac{1}{2} r_{n}\right)\left(2 K_{m}^{N}\right)^{-(m-1) / m}
$$

Consequently there exists a constant $A=A(N, m)$ such that if $S(P, r) \in \Gamma_{n}$ and $r>r_{n}$,

$$
F_{n}(P, r)>A r^{m}
$$

By Lemma 14 there will exist a surface $S_{0}$ with boundary $\supset L_{m-1}$ such that there exists a subsequence of the $n$ in which the $S_{n}$ converge to $S_{0}$. 


\section{Chapter 3}

I will now investigate the properties of a sequence of surfaces such as we have just constructed. Let then $\left\{S_{n}\right\}$ be any sequence of surfaces with boundary $\supset L_{m-1}$ such that, if $P \in S_{n}$ and $S(P, r) \cdot \Gamma=0$ then,

$$
\Lambda^{m} S_{n} \leqslant \mu\left(L_{m-1}\right)+\varepsilon_{n}
$$

and

$$
\Lambda^{m} S_{n} S(P, r) \geqslant A r^{m} \text { for } r>r_{n}
$$

where $A$ is fixed and $\varepsilon_{n} \rightarrow 0$ and $r_{n} \rightarrow 0$.

Suppose further that there exists a surface $S_{0}$ with boundary $\supset L_{m-1}$ such that $S_{n} \rightarrow S_{0}$. I will write

$$
\begin{aligned}
l_{n}(P, r) & =S_{n} s(P, r) \\
K_{n}(P, r) & =S_{n} S(P, r) \\
F_{n}(P, r) & =\int_{0}^{r} \Lambda^{m-1} l_{n}(P, t) d t
\end{aligned}
$$

and for convenience I shall write

$$
a_{1} \leqslant \varlimsup_{n \rightarrow \infty} a_{n} \leqslant a_{2} \text { to mean } a_{1} \leqslant \lim _{n \rightarrow \infty} a_{n} \leqslant \varlimsup_{n \rightarrow \infty} a_{n} \leqslant a_{2} .
$$

Let $\Gamma^{*}$ be the class of spheres $S(P, r)$ not meeting $\Gamma$ and with $P \in S_{0}$.

LEMMA $1^{*}$. If $S(P, r)$ does not meet $\Gamma$ then

$$
F_{n}(P, r) \leqslant \Lambda^{m} K_{n}(P, r) \leqslant \frac{r \Lambda^{m-1} l_{n}}{m} \frac{(P, r)}{r}+\varepsilon_{n} .
$$

This follows at once from Lemmas 4, 13 and 7, and (1) above.

Let now $\beta$ be the greatest lower bound of $\lim _{n \rightarrow \infty} \frac{\Lambda^{m} K_{n}(P, r)}{W_{m} r^{m}}$ taken over all $S(P, r) \in \Gamma^{*}$. LEM М $2^{*}$.

$$
\beta \geqslant \frac{A}{W_{m}}>0
$$

If $P \in S_{0}$ and $r^{\prime}<r$ then, for all sufficiently large $n$, we can choose $P_{n} \in S_{n}$ so near to $P$ that $K_{n}(P, r) \supset K_{n}\left(P_{n}, r^{\prime}\right)$.

Hence by (2) above $\beta>\frac{A r^{\prime m}}{W_{m} r^{m}}$ for all $r^{\prime}<r$, whence the lemma follows. 
LEMMA $3^{*}$. The greatest lower bound of $\lim _{n \rightarrow \infty} \frac{F_{n}(P, r)}{W_{m} r^{m}}$ taken over all $S(P, r) \in \Gamma^{*}$ is $\beta$.

By Lemma $1^{*}$

$$
\begin{aligned}
\underset{n \rightarrow \infty}{\lim _{n \rightarrow \infty} F_{n}(P, r)} & =\lim _{n \rightarrow \infty} \int_{0}^{r} \Lambda^{m-1} l_{n}(P, t) d t \\
& \geqslant \int_{0}^{r} \frac{\lim }{n \rightarrow \infty} \Lambda^{m-1} l_{n}(P, t) d t \geqslant \int_{0}^{r} \frac{\lim }{n \rightarrow \infty} \frac{m}{t}\left(\Lambda^{m} K_{n}(P, t)-\varepsilon_{n}\right) d t \\
& =\int_{0}^{r} \frac{m}{t} \frac{\lim }{n \rightarrow \infty} \Lambda^{m} K_{n}(P, t) d t \geqslant \int_{0}^{r} \frac{m}{t} \cdot \beta W_{m} t^{m} d t \geqslant \beta W_{m} r^{m}
\end{aligned}
$$

Thus $\beta$ is a lower bound of $\lim _{n \rightarrow \infty} \frac{F_{n}(P, r)}{W_{m} r^{m}}$ and since $F_{n}(P, r) \leqslant \Lambda^{m} K_{n}(P, r)$ it must be the greatest.

Lемма $4^{*}$. If $r_{1}>r_{2}$ and $S\left(P, r_{1}\right) \in \Gamma^{*}$ then

$$
\begin{aligned}
& \varlimsup_{\lim _{i \rightarrow \infty}} \frac{F_{n}\left(P, r_{1}\right)}{W_{m} r_{1}^{m}} \geqslant \overline{\lim }_{n_{i} \rightarrow \infty} \frac{F_{n}\left(P, r_{2}\right)}{W_{m} r_{2}^{m}} \\
& \varlimsup_{n_{i} \rightarrow \infty} \frac{F_{n}\left(P, r_{1}\right)}{W_{m} r_{1}^{m}} \geqslant \varlimsup_{n_{i \rightarrow \infty}} \frac{F_{n}\left(P, r_{2}\right)}{W_{m} r_{2}^{m}}
\end{aligned}
$$

for every subsequence $\left\{n_{i}\right\}$ of the integers.

\section{By Lemma $3^{*}$}

$$
F_{n}\left(P, r_{2}\right) \geqslant \frac{1}{2} \beta W_{m} r_{2}^{m} \text { for all large } n \text {. }
$$

Hence, for large $n$

$$
F_{n}(P, r) \geqslant \frac{1}{2} \beta W_{m} r_{2}^{m} \text { for all } r>r_{2},
$$

so that by Lemma $\mathbf{1}^{*}$, for almost all $r>r_{2}$,

$$
\begin{gathered}
F_{n}(P, r) \leqslant \frac{r}{m} \frac{d}{d r} F_{n}(P, r) \cdot\left(1+\frac{\varepsilon_{n}}{\frac{1}{2} \beta W_{m} r_{2}^{m}-\varepsilon_{n}}\right) . \\
\frac{\frac{d}{d r} F_{n}(P, r)}{F_{n} \overline{(P, r)}} \geqslant \frac{m}{r} \cdot\left(1-\frac{\varepsilon_{n}}{\frac{1}{2} \beta \cdot W_{m} r_{2}^{m}}\right) .
\end{gathered}
$$

Integrating from $r_{2}$ to $r_{1}$ 


$$
\log \frac{F_{n}\left(r_{1}\right)}{F_{n}\left(r_{2}\right)} \geqslant \log \frac{r_{1}^{m}}{r_{2}^{m}} \cdot\left(1-\frac{\varepsilon_{n}}{\frac{1}{2} \beta W_{m} r_{2}^{m}}\right)
$$

so that

$$
\frac{F_{n}\left(r_{1}\right) / W_{m} r_{1}^{m}}{F_{n}^{\prime}\left(r_{2}\right) / W_{m} r_{2}^{m}} \geqslant \exp \left\{\frac{-\varepsilon_{n}}{\frac{1}{2} \beta W_{m} r_{2}^{m}} \log \frac{r_{1}^{m}}{r_{2}^{m}}\right\}
$$

Whence the lemma follows.

Lemina $5^{*}$. If $\beta>0$ and $p>m$ then given $\eta>0$ there will exist $\varepsilon^{\prime}=\varepsilon^{\prime}(\eta, \beta, p, m)$, $\eta^{\prime}=\eta^{\prime}(\eta, \beta, p, m)$ and $\lambda=\lambda(\eta, \beta, p, m)$ such that if $S\left(P^{\prime}, r^{\prime}\right) \in \Gamma^{*}$, and $K_{0}\left(P^{\prime}, r^{\prime}\right)$ lies within $\eta^{\prime} r^{\prime}$ of some $p$-dimensional plane $\prod_{P}$, through $P^{\prime}$; and

$$
\begin{aligned}
& \beta \leqslant \varlimsup_{n \rightarrow \infty} \int_{0}^{r} \frac{\Lambda^{m-1} l_{n}(P, t) d t}{W_{m} r^{m}} \leqslant \beta+\varepsilon^{\prime} \\
& \beta \leqslant \varlimsup_{n \rightarrow \infty} \frac{\Lambda^{m} K_{n}(P, r)}{W_{m} r^{m}} \leqslant \beta+\varepsilon^{\prime}
\end{aligned}
$$

for every $S(P, r)$ of $\Gamma^{*}$ contained in $S\left(P^{\prime}, r^{\prime}\right)$; then there will exist $P \in S_{0}$ such that $K_{0}\left(P, \lambda r^{\prime}\right)$ lies within $\eta \lambda r^{\prime}$ of some $p-1$ dimensional plane through $P$, and $S\left(P, \lambda r^{\prime}\right)$ $\subset S\left(P^{\prime}, r^{\prime}\right)$.

We will first prove that if we write $R^{\prime}=2^{-(6 p-m+1) /(p-m)} p^{-p /(p-m)} r^{\prime}$ then there will exist $Q \in \prod_{P^{\prime}}$ such that $S\left(Q, R^{\prime}\right) \subset S\left(P^{\prime}, \frac{1}{4} r^{\prime}\right)$ and $K_{0}\left(Q, R^{\prime}\right)$ is null. For suppose not:- then if $S\left(Q_{i}, 2 R^{\prime}\right) \subset S\left(P^{\prime}, \frac{1}{4} r^{\prime}\right)$ and $Q_{i} \in \prod_{P^{\prime}}, K_{0}\left(Q_{i}, R^{\prime}\right)$ will not be null and hence will contain $Q_{i}^{\prime} \in S_{\mathbf{0}}$. Thus

$$
\begin{gathered}
S\left(Q_{i}^{\prime}, R^{\prime}\right) \subset S\left(Q_{i}, 2 R^{\prime}\right) \subset S\left(P^{\prime}, r^{\prime}\right) \text { and hence } \\
\lim _{n \rightarrow \infty} \Lambda^{m} K_{n}\left(Q_{i}, 2 R^{\prime}\right) \geqslant \lim _{n \rightarrow \infty} \Lambda^{m} K_{n}\left(Q_{i}^{\prime}, R^{\prime}\right) \geqslant \beta W_{m} R^{\prime m} .
\end{gathered}
$$

But we can find $\left\{r^{\prime} / 16 p R^{\prime}\right\}^{p}$ points $Q_{i}$ of $\prod_{P^{\prime}} S\left(P^{\prime}, \frac{1}{4} r^{\prime}-2 R^{\prime}\right)$ no two of which are within $4 R^{\prime}$ of each other, and hence

$$
\begin{aligned}
\left(\beta+\varepsilon^{\prime}\right) W_{m} 2^{-m} r^{\prime m} & \geqslant \lim _{n \rightarrow \infty} \Lambda^{m} K_{n}\left(P^{\prime}, \frac{1}{2} r^{\prime}\right) \\
& \geqslant \sum_{i} \lim _{n \rightarrow \infty} \Lambda^{m} K_{n}\left(Q_{i}, 2 R^{\prime}\right) \geqslant\left(\frac{r^{\prime}}{16 p R^{\prime}}\right)^{p} \cdot \beta W_{m} R^{\prime m} .
\end{aligned}
$$

This contradicts the definition of $R^{\prime}$, provided $\varepsilon^{\prime}<\beta$.

Suppose now that $S(Q, R)$ is the largest sphere with centre $Q$ having no points of $S_{\mathbf{0}}$ in its interior. Since $P^{\prime} \in S_{\mathbf{0}} S(Q, R) \subset S\left(P^{\prime}, \frac{1}{2} r^{\prime}\right)$. 
Suppose $P \in S_{0}$ lies at distance $R$ from $Q . K_{0}(P, r)$ will have no points inside $S(Q, R)$. Let $l_{n}=l_{n}(P, r)$ and let $l_{n}^{\theta}$ be the set of points of $l_{n}$ whose join to $P$ makes an angle not greater than $\theta$ with $Q P$ produced. Let $P_{0}$ be a point on $Q P$ produced and denote the distance $P P_{0}$ by $x<r$. Suppose $S_{n} \subset\left(S_{0}, \delta_{n}\right)$ where $\delta_{n} \rightarrow 0$. By Lemmas 7 and 13 we then have

$$
\begin{aligned}
& \left(\Lambda^{m-1} l_{n}-\Lambda^{m-1} l_{n}^{\theta}\right)\left(\sqrt{r^{2}+x^{2}+x r^{2} / R}+\delta_{n}\right) \\
& \quad+\Lambda^{m-1} l_{n}^{\theta} \sqrt{x^{2}+r^{2}-2 x r \cos \theta} \geqslant m \Lambda^{m} K_{n}(P, r)-m \varepsilon_{n} .
\end{aligned}
$$

Take $x=r_{0}^{2} / R, \cos \theta=2\left(r_{0} / R\right)^{1 / 3}$, and

$$
R / 2000>r_{0}>r>r_{0} \cdot\left(r_{0} / R\right)^{\frac{3}{2}}=r_{1} .
$$

Then after some manipulation we obtain

$$
\begin{aligned}
\Lambda^{m-1} l_{n}^{\theta} & \leqslant \frac{1}{r_{0}}\left(\frac{r_{0}}{R}\right)^{-9 / 8} \delta_{n} \Lambda^{m-1} l_{n} \\
& +\frac{1}{r_{0}} \cdot\left(\frac{r_{0}}{R}\right)^{-3 / s}\left\{r \Lambda^{m-1} l_{n}-m \Lambda^{m} K_{n}(P, r)+m \varepsilon_{n}+\frac{3}{2} r_{0} \cdot\left(\frac{r_{0}}{R}\right)^{s / 4} \Lambda^{m-1} l_{n}\right\} \\
& \leqslant \frac{1}{r_{0}}\left(\frac{r_{0}}{R}\right)^{-s / s} \delta_{n} \Lambda^{m-1} l_{n}+\frac{3}{2} \cdot\left(\frac{r_{0}}{R}\right)^{1 / 8} \Lambda^{m-1} l_{n}+\left(\frac{r_{0}}{R}\right)^{-9 / s}\left\{\Lambda^{m-1} l_{n}-\frac{m \Lambda^{m} K_{n}(P, r)}{r}+\frac{m \varepsilon_{n}}{r}\right\} .
\end{aligned}
$$

Hence $\quad \lim _{n \rightarrow \infty} \int_{r_{1}}^{r_{0}} \Lambda^{m-1} l_{n}^{\theta} d r \leqslant \frac{3}{2}\left(\frac{r_{0}}{R}\right)^{1 / s} \lim _{n \rightarrow \infty} \int_{r_{1}}^{r_{0}} \Lambda^{m-1} l_{n} d r$

$$
+\left(\frac{r_{0}}{R}\right)^{-\vartheta / 8}\left\{\lim _{n \rightarrow \infty} \int_{r_{1}}^{r_{0}} \Lambda^{m-1} l_{n} d r-\int_{r}^{r_{0}} \lim _{n \rightarrow \infty} \frac{m \Lambda^{m} K_{n}(P, r) d r}{r}\right\} .
$$

Thus since $m \geqslant 2$

$$
\begin{aligned}
\lim _{n \rightarrow \infty} \int_{r_{\mathrm{s}}}^{r_{s}} \Lambda^{m-1} l_{n}^{\theta} d r & \leqslant \frac{3}{2}\left(\frac{r_{0}}{R}\right)^{1 / s}\left(\beta+\varepsilon^{\prime}\right) W_{m} r_{0}^{m}+\left(\frac{r_{0}}{R}\right)^{-\% / s}\left\{\varepsilon^{\prime} W_{m} r_{0}^{m}+\beta W_{m} r_{1}^{m}\right\} \\
& \leqslant \frac{3}{2}\left(\frac{r_{0}}{R}\right)^{1 / s}\left(\beta+\varepsilon^{\prime}\right) W_{m} r_{0}^{m}+\left(\frac{r_{0}}{R}\right)^{-9 / s} \varepsilon^{\prime} W_{m} r_{0}^{m}+\left(\frac{r_{0}}{R}\right)^{3 / s} \beta W_{m} r_{0}^{m} .
\end{aligned}
$$

Let now $C_{\theta}$ be the set of points whose join to $P$ makes an angle not greater than $\theta$ with $Q P$ produced. Then by (4), Lemma 4, (3) and (7), 
$\lim _{n \rightarrow \infty} \Lambda^{m} K_{n}\left(P, r_{0}\right) C_{\theta}$

$$
\begin{aligned}
& \leqslant \varlimsup_{n \rightarrow \infty} \Lambda^{m} K_{n}\left(P, r_{0}\right)-\varlimsup_{n \rightarrow \infty} \Lambda^{m}\left\{K_{n}\left(P, r_{0}\right)-C_{\theta}\right\} \\
& \leqslant\left(\beta+\varepsilon^{\prime}\right) W_{m} r_{0}^{m}-\varlimsup_{n \rightarrow \infty} \int_{0}^{r_{0}} \Lambda^{m-1}\left(l_{n}-l_{n}^{\theta}\right) d r \\
& \leqslant\left(\beta+\varepsilon^{\prime}\right) W_{m} r_{0}^{m}-\varlimsup_{n \rightarrow \infty}\left\{\int_{0}^{r_{0}} \Lambda^{m-1} l_{n} d r-\int_{r_{2}}^{r_{0}} \Lambda^{m-1} l_{n}^{\theta} d r-\int_{0}^{r_{1}} \Lambda^{m-1} l_{n}^{\theta} d r\right\} \\
& \leqslant\left(\beta+\varepsilon^{\prime}\right) W_{m} r_{0}^{m}-\varlimsup_{n \rightarrow \infty} \int_{0}^{r_{0}} \Lambda^{m-1} l_{n} d r+\varlimsup_{n \rightarrow \infty} \int_{r_{1}}^{r_{0}} \Lambda^{m-1} l_{n}^{\theta} d r+\varlimsup_{n \rightarrow \infty} \int_{0}^{r_{1}} \Lambda^{m-1} l_{n} d r \\
& \leqslant\left(\beta+\varepsilon^{\prime}\right) W_{m} r_{0}^{m}-\beta W_{m} r_{0}^{m}+\frac{3}{2}\left(\frac{r_{0}}{R}\right)^{1 / s}\left(\beta+\varepsilon^{\prime}\right) W_{m} r_{0}^{m} \\
& \quad+\left(\frac{r_{0}}{R}\right)^{-9 / s} \varepsilon^{\prime} W_{m} r_{0}^{m}+\left(\frac{r_{0}}{R}\right)^{s_{s}} \beta W_{m} r_{0}^{m}+\left(\frac{r_{0}}{R}\right)^{3 / s}\left(\beta+\varepsilon^{\prime}\right) W_{m} r_{0}^{m} .
\end{aligned}
$$

Let $\Pi$ be the $N-1$ dimensional plane through $P$ orthogonal to $Q P$. Let $\lambda=r_{0} / 2 r^{\prime}$; then by the definition of $R^{\prime}$

$$
2 \lambda \cdot 2^{(6 p-m+1) /(p-m)} p^{p /(p-m)} \geqslant \frac{r_{0}}{R} \geqslant 2 \lambda
$$

I will now show that if $\lambda$ is chosen sufficiently small then we can choose $\varepsilon^{\prime}$ so small that $K_{0}\left(P, \frac{1}{2} r_{0}\right)=K_{0}\left(P, \lambda r^{\prime}\right)$ lies within $\frac{1}{4} \eta r_{0}=\frac{1}{2} \eta \lambda r^{\prime}$ of $\Pi$. For suppose than $P^{*} \in K_{0}\left(P, \frac{1}{2} r_{0}\right)$ lies further than $\frac{1}{4} \eta r_{0}$ from $\Pi$. $P^{*}$ will not lie in $S(Q, R)$ and hence if $\lambda$ is chosen so small that $r_{0} / 2 R<\eta, P^{*}$ must lie on the opposite side of $\prod$ to $Q$. Thus $S\left(P^{*}, \frac{1}{4} \eta r_{0}-\frac{1}{2} r_{0} \cos \theta\right) \subset S\left(P, r_{0}\right) C_{\theta}$ so that by (4) and (8)

$$
\begin{aligned}
W_{m} \beta r_{0}^{m}\left(\frac{1}{4} \eta-\frac{1}{2} \cos \theta\right)^{m} & \leqslant \lim _{n \rightarrow \infty} \Lambda^{m} K_{n}\left(P^{*}, \frac{\eta r_{0}}{4}-\frac{1}{2} r_{0} \cos \theta\right) \leqslant \frac{\lim }{n \rightarrow \infty} \Lambda^{m} K_{n}\left(P, r_{0}\right) C_{0} \\
& \leqslant \varepsilon^{\prime} W_{m} r_{0}^{m}+\frac{3}{2}\left(\frac{r_{0}}{R}\right)^{1 / s}\left(\beta+\varepsilon^{\prime}\right) W_{m} r_{0}^{m}+\left(\frac{r_{0}}{R}\right)^{-9 / s} \varepsilon^{\prime} W_{m} r_{0}^{m}+ \\
& +\left(\frac{r_{0}}{R}\right)^{3 / s} \beta W_{m} r_{0}^{m}+\left(\frac{r_{0}}{R}\right)^{3 / 4}\left(\beta+\varepsilon^{\prime}\right) W_{m} r_{0}^{m} .
\end{aligned}
$$

Now $\cos \theta=2\left(r_{0} / R\right)^{1 / s}$ so that we obtain

$$
\frac{1}{4} \eta \leqslant\left(\frac{r_{0}}{R}\right)^{1 / s}+\left\{\frac{\varepsilon^{\prime}}{\beta}\left(1+\left(\frac{r_{0}}{R}\right)^{-s / s}\right)+4\left(\frac{r_{0}}{R}\right)^{1 / s}\left(1+\frac{\varepsilon^{\prime}}{\beta}\right)\right\}^{1 / m}
$$


But by (9) above if $\lambda$ is sufficiently small we can choose $\varepsilon^{\prime}$ so small that (10) gives a contradiction. Hence $K_{0}\left(P, \lambda r^{\prime}\right)$ lies within $\frac{1}{2} \eta \lambda r^{\prime}$ of $\Pi$.

On the other hand $P \in S(Q, R) \subset S\left(P^{\prime}, \frac{1}{2} r^{\prime}\right)$ so that $K_{0}\left(P, \lambda r^{\prime}\right) \subset S\left(P^{\prime}, r^{\prime}\right)$. If $\Pi^{*}$ is the plane through $P$ parallel to $\Pi_{P^{\prime}}, \Pi^{*}$ will lie within $\eta^{\prime} r^{\prime}$ of $\Pi_{P^{\prime}}$ and hence $K_{0}\left(P, \lambda r^{\prime}\right)$ will lie within $2 \eta^{\prime} r^{\prime}$ of $\Pi^{*} . Q P$ is orthogonal to $\Pi$ and makes an angle with $\Pi^{*}$ whose sine is less than $\eta^{\prime} r^{\prime} / R \leqslant \eta^{\prime} 2^{(6 p-m+1) /(p-m)} p^{p /(m-p)}<\sin \pi / 4$, if $\eta^{\prime}$ is small. Hence $K_{0}\left(P, \lambda r^{\prime}\right)$ will lie within $\sqrt{\left(\frac{1}{2} \eta \lambda r^{\prime}\right)^{2}+\left(\frac{1}{2} \eta \lambda r^{\prime}+4 \eta^{\prime} r^{\prime}\right)^{2}}$ of the intersection of $\Pi$ and $\Pi^{*}$. If $\eta^{\prime}$ is taken small, this proves the lemma.

Lemma $6^{*}$. Given $\xi>0$ there will exist $\varepsilon_{0}=\varepsilon_{0}(\xi, \beta, m)$ and $v=v(\xi, \beta, m)$ such that if $S\left(P^{\prime}, r^{\prime}\right) \in \Gamma^{*}$ and

$$
\begin{aligned}
& \beta \leqslant \varlimsup_{n \rightarrow \infty} \int_{0}^{r} \frac{\Lambda^{m-1} l_{n}(P, t) d t}{W_{m} r^{m}} \leqslant \beta+\varepsilon_{0} \\
& \beta \leqslant \varlimsup_{n \rightarrow \infty} \frac{\Lambda^{m} K_{n}(P, r)}{W_{m} r^{m}} \leqslant \beta+\varepsilon_{0}
\end{aligned}
$$

for every $S(P, r) \subset S\left(P^{\prime}, r^{\prime}\right)$ with $P \in S_{0}$ then to each such sphere $S(P, r)$ there will correspond $P^{*} \in S_{0}$, and an m-dimensional plane $\Pi_{m}$ through $P^{*}$, such that

and

$$
S\left(P^{*}, v r\right) \subset S(P, r)
$$$$
K_{0}\left(P^{*}, v r\right) \supset\left(\Pi_{m}, \xi v r\right) .
$$

We need only note that $K_{0}(P, r)$ lies at zero distance from the plane $\Pi_{N}$ consisting of the whole space and then repeated applications of Lemma $5^{*}$ will give the result.

LEMmA $7^{*}$. Given $\theta>0, e_{\theta}>0$ and $\xi, 0<\xi<1-\cos \theta$. If $S(P, r)$ is a sphere such that for arbitrarily small $\delta>0$ we can find a non-overlapping set of spheres $\left\{S\left(P_{i}, r_{i}\right)\right\}$ such that

$$
\begin{aligned}
S\left(P_{i}, r_{i}\right) & \subset S(P, r), \\
r_{i} & <\delta, \\
\Lambda^{m} K_{0}\left(P_{i}, r_{i}\right) & >W_{m} r_{i}^{m}, \\
\sum W_{m} r_{i}^{m} & >e_{\theta}, \\
K_{0}\left(P_{i}, r_{i}\right) & \subset\left(\Pi_{i}, \xi r_{i}\right)
\end{aligned}
$$

and

where $\Pi_{i}$ is an m-dimensional plane through $P_{i}$ making an angle of more than $\theta$ with $P P_{i}$; then 


$$
\int_{0}^{r} \Lambda^{m-1} l_{0}(P, t) d t \leqslant \Lambda^{m} K_{0}(P, r)-e_{\theta}\left(\frac{1-\cos \theta-\xi}{2}\right)^{m} .
$$

Suppose $\Pi$ is an $m$-dimensional plane and that $Q_{1}$ and $Q_{2}$ are two points of $\Pi$ at a distance $\alpha$ apart; I then define $d(\alpha, \varrho, t)$ and $\sigma(\alpha, \varrho, t)$ to be respectively the diameter and $\Lambda^{m-1}$ measure of $\Pi S\left(Q_{1}, \varrho\right) s\left(Q_{2}, t\right)$. It follows immediately from the definition of the symbols concerned that

$$
\int_{0}^{t_{0}} \sigma(\alpha, \varrho, l) d t=\Lambda^{m} \Pi S\left(Q_{1}, \varrho\right) S\left(Q_{2}, t_{0}\right)
$$

and that for $\delta>\underline{\varrho}$

$$
\Lambda_{\delta}^{m-1} S\left(Q_{1}, \varrho\right) s\left(Q_{2}, t\right) \leqslant W_{m-1}\left\{\frac{1}{2} d(\alpha, \varrho, t)\right\}^{m-1} \leqslant \sigma(\alpha, \varrho, t) .
$$

We now choose a set of spheres $S\left(P_{\hat{g}}, r_{j}\right)$ such that $r_{j}<\delta$,

$$
K_{\mathbf{0}}(P, r)-\sum_{i} K_{0}\left(P_{i}, r_{i}\right) \subset \sum_{j} S\left(P_{j}, r_{j}\right)
$$

and

$$
\Lambda^{m}\left\{K_{0}(P, r)-\sum_{i} K_{0}\left(P_{i}, r_{i}\right)\right\}+\delta \geqslant \sum_{j} W_{m} r_{j}^{m}
$$

By (22) and (21)

$$
\int_{0}^{r} \Lambda_{\delta}^{m-1} l_{0}(P, t) S\left(P_{j}, r_{j}\right) d t \leqslant W_{m} r_{j}^{m}
$$

By (19), (22) and (21), if $P P_{i} \geqslant r_{i}$

$$
\begin{aligned}
& \int_{0}^{r} \Lambda_{\delta}^{m-1} l_{0}(P, t) S\left(P_{i}, r_{i}\right) d t \leqslant \int_{P P_{i}-r_{i} \cos \theta-r_{i} \xi}^{r} \sigma\left(P P_{i}, r_{i}, t\right) d t \\
& \quad \leqslant \int_{0}^{r} \sigma\left(P P_{i}, r_{i}, t\right) d t-\int_{0}^{P P_{i}-r_{i} \cos \theta-r_{i} \xi} \sigma\left(P P_{i}, r_{i}, t\right) d t \leqslant W_{m} r_{i}^{m}-W_{m}\left(\frac{1-\cos \theta-\xi}{2}\right)^{m} r_{i}^{m} .
\end{aligned}
$$

Now since the $S\left(P_{i}, r_{i}\right)$ are non-overlapping there is at most one $i=i_{0}$ such that $P P_{i}<r_{i} \leqslant \delta$ and by (22) and (21)

$$
\int_{0}^{r} \Lambda_{\delta}^{m-1} l_{0}(P, t) S\left(P_{i_{o}}, r_{i_{s}}\right) d t \leqslant W_{m} r_{i_{s}}^{m} \leqslant W_{m} \delta^{m} .
$$

Thus by (25), (26), (27), (24), (17) and (18)

3-60173032. Acta mathematica. 104. Imprimé le 23 septembre 1960 


$$
\begin{aligned}
& \int_{0}^{r} \Lambda_{\delta}^{m-1} l_{0}(P, t) d t=\int_{0}^{r} \Lambda_{\delta}^{m-1}\left\{\sum_{j} S\left(P_{j}, r_{j}\right)+\sum_{i} S\left(P_{i}, r_{i}\right)\right\} l_{0}(P, t) d t \\
& \quad \leqslant \sum_{j} \int_{0}^{r} \Lambda_{\delta}^{m-1} l_{0}(P, t) S\left(P_{j}, r_{j}\right) d t+\sum_{i} \int_{0}^{r} \Lambda_{\delta}^{m-1} l_{0}(P, t) S\left(P_{i}, r_{i}\right) d t \\
& \quad \leqslant \sum_{j} W_{m} r_{j}^{m}+\sum_{i} W_{m} r_{i}^{m}\left\{1-\left(\frac{1-\cos \theta-\xi}{2}\right)^{m}\right\}+W_{m} \delta^{m} \\
& \quad \leqslant \Lambda^{m}\left\{K_{0}(P, r)-\sum_{i} K_{0}\left(P_{i}, r_{i}\right)\right\}+\delta+W_{m} \delta^{m}+\Lambda^{m} \sum_{i} K_{0}\left(P_{i}, r_{i}\right)-e_{\theta} \cdot\left(\frac{1-\cos \theta-\xi}{2}\right)^{m} \\
& \quad \leqslant \Lambda^{m} K_{0}(P, r)-e_{\theta} \cdot\left(\frac{1-\cos \theta-\xi}{2}\right)^{m}+\delta+W_{m} \delta^{m} .
\end{aligned}
$$

Thus, letting $\delta \rightarrow 0$, we obtain (20) which proves the lemma.

Take $\xi>0$ and define $\varepsilon$ and $v$ as in Lemma $6^{*}$, where we may clearly suppose that $\varepsilon<\beta$. There will exist $S\left(P_{1}, r_{1}\right) \in \Gamma^{*}$ such that

$$
\beta \leqslant \lim _{n \rightarrow \infty} \frac{\Lambda^{m} K_{n}\left(P_{1}, r_{1}\right)}{W_{m} r_{1}^{m}} \leqslant \beta+\frac{\varepsilon^{2}}{50 \beta} .
$$

Thus there will be a subsequence $\left\{n_{i}\right\}$ of the integers such that $\lim _{i \rightarrow \infty} \Lambda^{m} K_{n_{i}}\left(P_{1}, r_{1}\right)$ exists and

$$
\beta \leqslant \lim _{i \rightarrow \infty} \frac{\Lambda^{m} K_{n_{i}}\left(P_{1}, r_{1}\right)}{W_{m} r_{1}^{m}} \leqslant \beta+\frac{\varepsilon^{2}}{50 \beta}
$$

If now $r_{2}<\frac{1}{3} r_{1}$ is sufficiently small and $P \in S_{0} S\left(P_{1}, r_{2}\right)$

$$
\begin{gathered}
\beta \leqslant \varlimsup_{i \rightarrow \infty} \frac{\Lambda^{m} K_{n_{i}}\left(P, r_{1}-r_{2}\right)}{W_{m}\left(r_{1}-r_{2}\right)^{m}} \leqslant \beta+\frac{\varepsilon^{2}}{25 \beta}, \\
\beta \leqslant \varlimsup_{i \rightarrow \infty} \frac{F_{n_{i}}\left(P, r_{1}-r_{2}\right)}{W_{m}\left(r_{1}-r_{2}\right)^{m}} \leqslant \beta+\frac{\varepsilon^{2}}{25 \beta} .
\end{gathered}
$$

and hence

Thus by Lemma $4^{*}$, if $t<r_{1}-r_{2}$,

$$
\beta \leqslant \varlimsup_{\overline{i \rightarrow \infty}} \frac{F_{n_{i}}(P, t)}{W_{m} t^{m}} \leqslant \beta+\frac{\varepsilon^{2}}{25 \beta} .
$$

I will now show that for $r<\frac{1}{2}\left(r_{1}-r_{2}\right)$

$$
\beta \leqslant \varlimsup_{i \rightarrow \infty} \frac{\Lambda^{m} K_{n_{i}}(P, r)}{W_{m} r^{m}} \leqslant \beta+\varepsilon,
$$

for, if not, there will exist arbitrarily large $n_{i}$ such that 


$$
\frac{\Lambda^{m} K_{n_{i}}(P, r)}{W_{m} r^{m}}>\beta+\frac{1}{2} \varepsilon
$$

hence for all $t$ such that

$$
\begin{gathered}
r<t<r\left(\frac{\beta+\frac{1}{2} \varepsilon}{\beta+\frac{1}{4} \varepsilon}\right)^{\frac{1}{m}}=r^{\prime}<r_{1}-r_{2} \\
\frac{\Lambda^{m} K_{n_{i}}(P, t)}{W_{m} t^{m}}>\beta+\frac{1}{4} \varepsilon
\end{gathered}
$$

so that by Lemma $1^{*}$

$$
\Lambda^{m-1} l_{n_{i}}(P, t) \geqslant W_{m}\left(\beta+\frac{1}{4} \varepsilon\right) m t^{m-1}-\frac{m \varepsilon_{n}}{t}
$$

and hence

$$
\begin{aligned}
\int_{0}^{r^{\prime}} \Lambda^{m-1} l_{n_{i}}(P, t) d t & =\int_{0}^{r} \Lambda^{m-1} l_{n_{i}}(P, t) d t+\int_{r}^{r^{\prime}} \Lambda^{m-1} l_{n_{i}}(P, t) d t \\
& \geqslant \int_{0}^{r} \Lambda^{m-1} l_{n_{i}}(P, t) d t+W_{m}\left(\beta+\frac{1}{4} \varepsilon\right)\left(r^{m}-r^{m}\right)-m \varepsilon_{n_{i}} \log \frac{r^{\prime}}{r} .
\end{aligned}
$$

Thus letting $n_{i} \rightarrow \infty$, and using (32)

$$
\left(\beta+\frac{\varepsilon^{2}}{25 \beta}\right) r^{\prime m} \geqslant \beta r^{m}+\left(\beta+\frac{1}{4} \varepsilon\right)\left(r^{\prime m}-r^{m}\right)
$$

which since $\varepsilon<\beta$ gives a contradiction with (34).

It follows from (33) that

$$
\beta \leqslant \varlimsup_{i \rightarrow \infty} \frac{F_{n_{i}}(P, r)}{W_{m} r^{m}} \leqslant \beta+\varepsilon .
$$

(33) and (35) hold whenever $r<\frac{1}{2}\left(r_{1}-r_{2}\right)$ and $P \in S_{0} S\left(P_{1}, r_{2}\right)$ which will certainly be the case if $S(P, r) \in \Gamma^{*}$ and $S(P, r) \subset S\left(P_{1}, r_{2}\right)$. We may therefore apply Lemma $6^{*}$ to conclude that to each such $S(P, r)$ there will correspond $P^{*} \in S_{0}$ and an $m$-dimensional plane $\Pi_{m}(P)$ such that

$$
S\left(P^{*}, v r\right) \subset S(P, r)
$$

and

$$
K_{0}\left(P^{*}, v r\right) \subset\left(\Pi_{m}(P), \xi v r\right) \text {. }
$$

Hence for large $n_{i}$

$$
K_{n_{i}}\left(P^{*}, v r\right) \subset\left(\Pi_{m}(P), 2 \xi v r\right) .
$$

Moreover for large $n_{i}$ by (33)

$$
\Lambda^{m} K_{n i}\left(P^{*}, \frac{1}{2} v r\right)>\frac{1}{2} \beta W_{m} 2^{-m} v^{m} r^{m}
$$

and by (35)

$$
F_{n_{i}}\left(P^{*}, v r\right)<(\beta+2 \varepsilon) W_{m} v^{m} r^{m}
$$


Hence we can find $\varrho_{i}, \frac{1}{2} v r<\varrho_{i}<v r$ such that

$$
\Lambda^{m-1} l_{n_{i}}\left(P^{*}, \varrho_{i}\right) \leqslant 2(\beta+2 \varepsilon) W_{m} v^{m-1} r^{m-1} .
$$

Then by Lemma (12) in view of equation (1) together vith Lemma (13) either

or else

$$
\begin{aligned}
\Lambda^{m} K_{n_{i}}\left(P^{*}, \varrho_{i}\right) & \geqslant W_{m} \varrho_{i}^{m}-\frac{2^{2 m} W_{m}}{W_{m-1}} \cdot 2 \xi v r \cdot \Lambda^{m-1} l_{n_{i}}\left(P^{*}, \varrho_{i}\right) \\
& \geqslant W_{m} \varrho_{i}^{m}-\frac{2^{2 m+2} W_{m}^{2}}{W_{m-1}} \cdot \xi(\beta+2 \varepsilon) v^{m} r^{m}
\end{aligned}
$$

$$
\begin{aligned}
\frac{1}{2} \beta W_{m} & \frac{v^{m} r^{m}}{2^{m}}<\Lambda^{m} K_{n_{i}}\left(P^{*}, \frac{1}{2} v r\right) \leqslant \Lambda^{m} K_{n_{i}}\left(P^{*}, \varrho_{i}\right) \\
& \leqslant \frac{2^{2 \cdot n} W_{m}}{W_{m-1}} \cdot 2 \xi v r \cdot \Lambda^{m-1} l_{n_{i}}\left(P^{*}, \varrho_{i}\right)+\varepsilon_{n_{i}} \leqslant \frac{2^{2 m+2} W_{m}^{2}}{W_{m-1}} \cdot \xi(\beta+2 \varepsilon) v^{m} r^{m}+\varepsilon_{n_{i}} .
\end{aligned}
$$

The latter is impossible if $\xi$ is small and hence (41) must hold.

Letting $i \rightarrow \infty$ through a subsequence such that $\varrho_{i}$ tends to a limit $\varrho$ we have $\frac{1}{2} v r \leqslant \varrho \leqslant v r$ and for each $\delta>0$

$$
\varlimsup_{n_{i} \rightarrow \infty} K_{n_{i}}\left(P^{*}, \underline{\varrho}+\delta\right) \geqslant \boldsymbol{W}_{m} \varrho^{m}-\frac{2^{2 m+2} W_{m}^{2}}{W_{m-1}} \xi(\beta+2 \varepsilon) v^{m} r^{m}
$$

using (33) and letting $\delta \rightarrow 0$ we find $\beta+\varepsilon \geqslant 1-\frac{2^{3 m+2} W_{m}}{W_{m-1}}(\beta+2 \varepsilon) \xi$. Since $\xi$ and $\varepsilon$ may be chosen arbitrarily small we thus find that

$$
\beta \geqslant 1 \text {. }
$$

Suppose now that we have a finite set of non-overlapping spheres $S\left(P_{i}, r_{i}\right) \in \Gamma^{*}$, and contained in an open set $G$. Then

$$
\sum_{i} W_{m} r_{i}^{m} \leqslant \sum_{i} \lim _{n \rightarrow \infty} \Lambda^{m} K_{n}\left(P_{i}, r_{i}\right) \leqslant \lim _{n \rightarrow \infty} \Lambda^{m} \sum_{i} K_{n}\left(P_{i}, r_{i}\right) \leqslant \lim _{n \rightarrow \infty} \Lambda^{m} S_{n} G .
$$

If $\delta^{\prime}>\delta>0$ we will be able to find a finite set of points $\left\{P_{i}\right\}$ belonging to $S_{0}-\left(\Gamma, \delta^{\prime}\right)$ and such that $S_{0}-\left(\Gamma, \delta^{\prime}\right) \subset \sum_{i} S\left(P_{i}, \delta\right)$ and $P_{i} P_{i^{\prime}}>\delta$ if $i \neq i^{\prime}$

Each sphere $S\left(P_{i}, \delta\right)$ will be met by less than $5^{N}$ others. We may divide the set of spheres $S\left(P_{i}, \delta\right)$ into subsets $\sum_{j}$ such that each $\sum_{j}$ consists of disjoint spheres and if $S\left(P_{i}, \delta\right)$ does not belong to any $\sum_{j}\left(j=1,2 \ldots j_{0}\right)$ then it must meet at least one sphere from each of these sets. Clearly there are at most $5^{N}$ sets $\sum_{j}$ and hence by (44) above and (1)

$$
\sum_{i} W_{m} \delta^{m} \leqslant 5^{N} \mu\left(L_{m-1}\right)
$$


Consequently

$$
\Lambda_{\delta}^{m}\left(S_{0}-\left(\Gamma, \delta^{\prime}\right)\right) \leqslant 5^{N} \mu\left(L_{m-1}\right) .
$$

Letting first $\delta$ and then $\delta^{\prime} \rightarrow 0$ we find that

$$
\Lambda^{m}\left(S_{0}-\Gamma\right)<\infty
$$

Suppose now that $G \cdot \Gamma=0$. By Lemma 1 we may cover almost all of $S_{0} G$ by a set of arbitrarily small spheres $S\left(P_{i}, r_{i}\right)$ contained in $G$ and belonging to $\Gamma^{*}$, and by (44) we then have

$$
\sum_{i} W_{m} r_{i}^{m} \leqslant \lim _{n \rightarrow \infty} \Lambda^{m} S_{n} G
$$

whence by the definition of $\Lambda^{m} X$

$$
\Lambda^{m} S_{0} G \leqslant \lim _{n \rightarrow \infty} \Lambda^{m} S_{n} G
$$

In particular

$$
\Lambda^{m} S_{0}=\Lambda^{m}\left(S_{0}-\Gamma\right)+\Lambda^{m}(\Gamma) \leqslant \lim _{n \rightarrow \infty} \Lambda^{m}\left(S_{n}-\Gamma\right)+\Lambda^{m} \Gamma \leqslant \lim _{n \rightarrow \infty} \Lambda^{m} S_{n}=\mu\left(L_{n-1}\right) .
$$

Consequently by the definition of $\mu\left(L_{m-1}\right)$

$$
\Lambda^{m} S_{0}=\mu\left(L_{m-1}\right)
$$

Taking this in conjunction with Lemma 15 we have thus proved the following:

THEOREM. If $\Gamma$ is any closed set and $L_{m-1} \subset H_{m-1}(\Gamma)$ then there will exist a proper surface $S_{0}$ with boundary $\supset L_{m-1}$ uhich minimizes the area in the class of all such surfaces.

On the other hand suppose $S_{0}$ is any proper surface with boundary $\supset L_{m-1}$ such that $\Lambda^{m} S_{0}=\mu\left(L_{m-1}\right)$. Suppose $S(P, r) \in \Gamma^{*}$, then $\Lambda^{m-1} S_{0} s(P, r) \neq 0$ for if it were zero $H_{m-1}\left(S_{0} s(P, r)\right)$ would be null by Lemma $17 \mathrm{~A}$ and consequently, by Lemmas 13 and $1 \mathrm{~A}, \overline{S_{0}-S(P, r)}$ would be a surface with boundary $\supset L_{m-1}$ so that $S_{0}$ could not be a proper surface.

By Lemmas 8 and 13

$$
\Lambda^{m} K_{0}(P, r) \leqslant K_{m}^{N}\left\{\Lambda^{m-1} l_{0}(P, r)\right\}^{m /(m-1)}
$$

and hence by Lemma 4

$$
\int_{0}^{r} \Lambda^{m-1} l_{0}(P, t) d t \leqslant K_{m}^{N}\left\{\Lambda^{m-1} l_{0}(P, r)\right\}^{m(m-1)} .
$$

Hence

$$
\frac{\frac{d}{d r} F_{0}(P, r)}{\left\{\boldsymbol{F}_{\mathbf{0}}(P, r)\right\}^{(m-1) / m}} \geqslant\left\{K_{m}^{N}\right\}^{-(m-1) / m} .
$$


Integrating

and hence

$$
\left\{\boldsymbol{F}_{\mathbf{0}}(P, r)\right\}^{1 / m} \geqslant\left\{K_{m}^{N}\right\}^{-(m-1) / m} r
$$

$$
\Lambda^{m} K_{0}(P, r) \geqslant F_{0}(P, r) \geqslant\left\{K_{m}^{N}\right\}^{-(m-1)} r^{m} .
$$

It follows that if we put $S_{n}=S_{0}$ for every $n$ we will obtain a sequence satisfying our hypothesis (1) and (2) and consequently we may apply the subsequent results.

I will now prove the following:

THEOREM. If $S_{0}$ is a proper minimal surface and $P_{1} \in S_{0}-\Gamma$ is a point such that

$$
\lim _{r \rightarrow 0} \frac{\Lambda^{m} K_{0}\left(P_{1}, r\right)}{W_{m} r^{m}} \leqslant 1
$$

and $\varepsilon^{*}>0$ then there will exist $R_{1}>0, R_{0}>0$ such that to each point $X \in S_{0} S\left(P_{1}, R_{0}\right)$ and each $R<R_{0}$ there will correspond a plane ${ }_{R} \sum_{X}$ through $X$ such that

$$
\begin{aligned}
& S_{0} S\left(P_{1}, R_{1}\right) S(X, R) \subset\left({ }_{R} \sum_{X}, \varepsilon^{*} R\right) S(X, R) \\
& { }_{R} \sum_{X} S(X, R) \subset\left(S_{0} S\left(P_{1}, R_{1}\right), \varepsilon^{*} R\right) S(X, R)
\end{aligned}
$$

and there will exist a plane $\sum$ through $P$, such that

$$
S_{0} S\left(P_{1}, R_{1}\right) \subset\left(\Sigma, \varepsilon^{*} R_{0}\right) .
$$

Take $\xi$ such that

$$
160(m+1)(m+2) \xi^{\frac{1}{2}}<\varepsilon^{*}
$$

and let $\varepsilon_{0}=\varepsilon_{0}(\xi), v=v(\xi)$ be defined as in Lemma $6^{*}$. Take $\varepsilon<\varepsilon_{0}$ such that

$$
(8 m \varepsilon)^{1 / m}<\frac{1}{4} v \xi
$$

and $\theta$ such that

$$
1-\cos \theta=3 \xi
$$

By (49) and (43), (28) will hold for some sufficiently small $r_{1}$ and hence there will exist $r_{2}>0$ such that, by (33), (35), (36) and (37) if $P, P^{\prime} \in S_{0}$ and

$$
S(P, r) \subset S\left(P^{\prime}, r^{\prime}\right) \subset S\left(P_{1}, r_{2}\right)
$$

then: $\quad 1 \leqslant \frac{\int_{0}^{r} l_{0}(P . t) d t}{W_{m} r^{m}} \leqslant \frac{\Lambda^{m} K_{0}(P, r)}{W_{m} r^{m}} \leqslant 1+\varepsilon$

and there will exist $P^{*} \in S_{0}$ and an $m$-dimensional plane $\Pi_{m}(P)$ through $P^{*}$ such that

$$
S\left(P^{*}, v r\right) \subset S(P, r)
$$


and

$$
K_{0}\left(P^{*}, v r\right) \subset\left(\Pi_{m}(P), \xi v r\right) .
$$

Note first that by (51), (52)

$$
\frac{1}{2(m+3)(1+\varepsilon)} W_{m}\left(\frac{r^{\prime}}{8}\right)^{m} v^{m}\left(\frac{1-\cos \theta-\xi}{2}\right)^{m}+W_{m}\left(\frac{r^{\prime}}{2}\right)^{m}>(1+\varepsilon) W_{m}\left(\frac{r^{\prime}}{2}\right)^{m} .
$$

Suppose now that $Q_{1} \ldots Q_{m+2}$ are any $m+2$ points of $K_{0}\left(P^{\prime}, \frac{1}{4} r^{\prime}\right)$. Take $\delta>0$. By Lemma 1 we can find a finite set of non-overlapping spheres $S\left(P_{\delta i}, \varrho_{\delta i}\right)$ contained in $S\left(P^{\prime}, \frac{1}{4} r^{\prime}\right)$ such that $\varrho_{\delta i}<\delta, P_{\delta i} \in S_{0}$ and

$$
\sum_{i} \Lambda^{m} K_{0}\left(P_{\delta i}, \varrho_{\delta i}\right) \geqslant \frac{1}{2} \Lambda^{m} K_{0}\left(P^{\prime}, \frac{1}{8} r^{\prime}\right)
$$

and hence

$$
\sum_{i} W_{m} \varrho_{\delta i}^{m} \geqslant \frac{1}{2(1+\varepsilon)} \Lambda^{m} K_{0}\left(P^{\prime}, \frac{1}{8} r^{\prime}\right) \geqslant \frac{W_{m}}{2(1+\varepsilon)}\left(\frac{r^{\prime}}{8}\right)^{m}
$$

Moreover for each $j \leqslant m+2$ and each $i$

$$
S\left(P_{\delta i}, \varrho_{\delta i}\right) \subset S\left(P^{\prime}, \frac{1}{4} r^{\prime}\right) \subset S\left(Q_{j}, \frac{1}{2} r^{\prime}\right) \subset S\left(F^{\prime}, r^{\prime}\right) .
$$

We then obtain a set of spheres $S\left(P_{\delta i}^{*}, v \varrho_{\delta i}\right)$ such that $P_{\delta i}^{*} \in S_{0}$ and there exists a plane $\Pi_{m}\left(P_{\delta i}\right)$ such that $P_{\delta i}^{*} \in K_{\mathbf{0}}\left(P_{\delta i}, \varrho_{\delta i}\right)$ and

$$
K_{\mathbf{0}}\left(P_{\delta i}^{*}, v \varrho_{\delta i}\right) \subset\left(\Pi_{m}\left(P_{\delta i}\right), \xi v \varrho_{\delta i}\right) .
$$

Let $\sigma_{j \delta}$ be the set of $i$ such that $P_{\delta i}^{*} Q_{j}$ makes angle of more than $\theta$ with $\Pi_{m}\left(P_{\delta i}\right)$. I will now apply Lemma $7^{*}$ taking

$$
e_{\theta}=\frac{W_{m} v^{m}}{2(m+3)(1+\varepsilon)}\left(\frac{r^{\prime}}{8}\right)^{m}
$$

Suppose there exists arbitrarily small $\delta$ such that

then by $(20)$

$$
\sum_{i \in \sigma_{j \delta}} W_{m} \varrho_{i \delta}^{m} v^{m} \geqslant e_{\theta}
$$

$$
\int_{0}^{\frac{1}{2} r^{\prime}} \Lambda^{m-1} l_{0}\left(Q_{j}, t\right) d t \leqslant \Lambda^{m} K_{0}\left(Q_{j}, \frac{1}{2} r^{\prime}\right)-e_{\theta}\left(\frac{1-\cos \theta-\xi}{2}\right)^{m}
$$

and by (53) this contradicts (56).

Thus for all sufficiently small $\delta$

$$
\sum_{i \in \sigma_{j \delta}} W_{m} \varrho_{i \delta}^{m} v^{m}<\frac{W_{m} v^{m}}{2(m+3)(1+\varepsilon)}\left(\frac{r^{\prime}}{8}\right)^{m}
$$


and hence, by (57), since there are only $m+2$ points $Q_{j}$, we may choose $\delta_{0}$ so small that there exists $i_{0}$ not belonging to any $\sigma_{j \delta_{0}}$. Thus $P_{\delta_{0} i_{0}}^{*} Q_{j}$ makes an angle of less than $\theta$ with $\Pi_{m}\left(P_{\delta_{0} i_{0}}\right)$ for each $j$. We have therefore shown, since $1-\cos \theta=3 \xi$, that given any $m+2$ points $Q_{j}$ of $K_{0}\left(P^{\prime}, \frac{1}{4} r^{\prime}\right)$ we can find a plane $\Pi_{m}$ such that each $Q_{j}$ lies within $\frac{1}{2} r^{\prime} \sin \theta<\frac{5}{4} r^{\prime} \xi^{\frac{1}{2}}$ of $\Pi_{m}$.

Suppose now that $Q_{1}^{*} \ldots Q_{m+1}^{*}$ are the vertices of the $m$-dimensional tetrahedron of largest area with vertices in $K_{0}\left(P^{\prime}, \frac{1}{4} r^{\prime}\right)$, and let this area be $A$. Let $Q$ be any other point of $K_{0}\left(P^{\prime}, \frac{1}{4} r^{\prime}\right)$. Let $\Pi_{m}$ be the plane defined above corresponding to $Q_{1}^{*} \ldots Q_{m+1}^{*}, Q$. The area of each face of the tetrahedron $\Delta$ with vertices $Q_{1}^{*} \ldots Q_{m+1}^{*}, Q$ will be at most $A$ and hence the area of the projection of $\Delta$ onto $\Pi_{m}$ will be at most $(m+2) A$, and so the volume of $\Delta$ cannot exceed $\frac{5}{2}(m+2) A r^{\prime} \xi^{\frac{1}{2}}$. Thus $Q$ must lie within $\frac{5}{2}(m+1)(m+2) r^{\prime} \xi^{\frac{k}{2}}<\varepsilon^{*} r^{\prime} / 64$ of the plane of $Q_{1}^{*} \ldots Q_{m+1}^{*}$.

This has been proved for every point of $K_{0}\left(P^{\prime}, \frac{1}{4} r^{\prime}\right)$ and hence for each $S\left(P^{\prime}, r^{\prime}\right)$ $\subset S\left(P_{1}, r_{2}\right)$ we can find a plane $\Pi_{m}\left(P^{\prime}, r^{\prime}\right)$ passing through $P^{\prime}$ such that

$$
K_{0}\left(P^{\prime}, \frac{1}{4} r^{\prime}\right) \subset\left(\Pi_{m}\left(P^{\prime}, r^{\prime}\right), \frac{\varepsilon^{*} r^{\prime}}{32}\right)
$$

By (53) there will exist $x, \frac{1}{4} r^{\prime}>x>\frac{1}{8} r^{\prime}$ such that

$$
\begin{aligned}
& \Lambda^{m} K_{0}\left(P^{\prime}, x\right) \geqslant W_{m} x^{m}, \\
& \Lambda^{m-1} l_{0}\left(P^{\prime}, x\right) \leqslant(1+\varepsilon) W_{m}\left(\frac{1}{4} r^{\prime}\right)^{m} / \frac{1}{8} r^{\prime}
\end{aligned}
$$

so that if $\varepsilon^{*}$ is small

$$
\Lambda^{m} K_{0}\left(P^{\prime}, x\right)>\Lambda^{m-1} l_{0}\left(P^{\prime}, x\right) \cdot 2^{2 m} \frac{W_{m}}{W_{m-1}} \cdot \frac{\varepsilon^{*} x}{4} .
$$

But $S_{0}$ is a surface of minimum area so that by $(60),(63)$ and Lemmas (13) and (12) the projection of $K_{0}\left(P^{\prime}, x\right)$ onto $\Pi_{m}\left(P^{\prime}, r^{\prime}\right)$ will contain $\Pi_{m}\left(P^{\prime}, r^{\prime}\right) S\left(P^{\prime}, x\left(1-\frac{1}{4} \varepsilon^{*}\right)\right)$. By $(60)$ the projection of $K_{0}\left(P^{\prime}, x\right)-K_{0}\left(P^{\prime}, \frac{1}{8} r^{\prime}\right)$ onto $\Pi_{m}\left(P^{\prime}, r^{\prime}\right)$ will not meet $S\left(P^{\prime}, \frac{1}{8} r^{\prime}-\frac{\varepsilon^{*} r^{\prime}}{32}\right)$ and hence

$$
\left(K_{0}\left(P^{\prime}, \frac{1}{8} r^{\prime}\right), \varepsilon^{*} \frac{r^{\prime}}{8}\right) \supset\left(K_{0}\left(P^{\prime}, \frac{1}{8} r^{\prime}\right), \frac{\varepsilon^{*} r^{\prime}}{32}+\frac{1}{4} \varepsilon^{*} x\right) \supset \Pi_{m}\left(P^{\prime}, r^{\prime}\right) S\left(P^{\prime}, \frac{1}{8} r^{\prime}\right)
$$

Write $R_{1}=r_{2} / 4, R_{0}=r_{2} / 16$

$$
\sum=\Pi_{m}\left(P_{1}, 4 R_{1}\right), \quad{ }_{R} \sum_{X}=\Pi_{m}(X, 8 R) .
$$


If $X \in K_{0}\left(P_{1}, R_{0}\right)$ and $R<R_{0}$

and

$$
\begin{gathered}
S(X, R) \subset S\left(P_{1}, R_{1}\right), \\
S(X, 8 R) \subset S\left(P_{1}, r_{2}\right)
\end{gathered}
$$$$
S\left(P_{1}, 4 R_{1}\right) \subset S\left(P_{1}, r_{2}\right)
$$

whence the theorem follows from (60) and (64).

Thus taking the above in conjunction with the theorem of Chapter 4 the main theorem of this paper is established.

\section{Chapter 4}

Lемма 1. If $\sum$ and $\Sigma$ ' are m-planes through a point $O$ and $\Pi$, $\Pi$ ' are $N-m$. planes through $O$ orthogonal to $\Sigma$ and $\Sigma$ respectively, then if

$\begin{array}{ll} & \Sigma^{\prime} S(0,1) \subset(\Sigma, \beta), \\ \text { then } & \Pi S(0,1) \subset\left(\Pi^{\prime}, 2 \beta\right) .\end{array}$

Let $X$ be a point of $\Pi$ such that $|O X|=1$. Let $X^{*}$ be the projection of $X$ onto $\Sigma^{\prime}$. Then $X^{*}$ will be within $\beta\left|O X^{*}\right|$ of $\Sigma$ and hence

$$
\left|X X^{*}\right|+\beta\left|O X^{*}\right| \geqslant|O X| \text {. }
$$

That is

$$
\left(1-\left|O X^{*}\right|^{2}\right)^{\frac{1}{2}}+\beta\left|O X^{*}\right| \geqslant 1,
$$

whence

$$
\left|O X^{*}\right| \leqslant 2 \beta /\left(1+\beta^{2}\right) \leqslant 2 \beta \text {. }
$$

Since $X X^{*}$ is orthogonal to $\Sigma^{\prime}$ the distance of $X$ from $\Pi^{\prime}$ is equal to $\left|O X^{*}\right|$ and the lemma follows.

Lемма 2. If $\left\{h_{k}^{1}\right\},\left\{h_{k}^{2}\right\}$ are two sets of $N$ orthogonal unit vectors, $(k=1, \ldots, N)$, such that

$$
\left|h_{k}^{1}-h_{k}^{2}\right|<\beta \quad(k=1, \ldots, N)
$$

and $\Sigma_{1}, \Sigma_{2}$ are two m-planes, through a common point $O$, containing $\left\{h_{k}^{1}\right\}$ and $\left\{h_{k}^{2}\right\}$ $(k=1, \ldots, m)$ respectively, then

$$
\Sigma_{1} \cdot S(0,1) \subset\left(\Sigma_{2}, N \beta\right)
$$

Suppose $P \in \Sigma_{1},|O P|=1$, then the vector $O P$ will be of the form $\sum_{1}^{m} \lambda_{k} h_{k}^{1}$, where $\sum \lambda_{k}^{2}=1$. Let $P^{\prime}$ be the point of $\sum_{2}$ such that $O P^{\prime}=\sum_{1}^{m} \lambda_{k} h_{k}^{2}$, then 


$$
\left|P P^{\prime}\right|=\left|O P-O P^{\prime}\right|=\left|\sum_{1}^{m} \lambda_{k}\left(h_{k}^{\mathbf{1}}-h_{k}^{2}\right)\right| \leqslant \beta \sum_{1}^{m}\left|\lambda_{k}\right| \leqslant N \beta \text {. }
$$

LEMMA 3. Suppose $h_{1}, \ldots, h_{N}$ and $g_{1}, \ldots, g_{N}$ are two sets of orthogonal unit vectors, and that $\Sigma_{h}$ and $\Sigma_{g}$ are $m$-planes containing $h_{1}, \ldots, h_{m}$ and $g_{1}, \ldots, g_{m}$ respectively and passing through a point $O ; \sum_{h}^{\prime}$ and $\sum_{g}^{\prime}$ are two planes through $O$ such that

$$
\begin{aligned}
& \sum_{h}^{\prime} S(0,1) \subset\left(\sum_{h}, d\right) \\
& \sum_{g}^{\prime} S(0,1) \subset\left(\sum_{g}, d\right) \\
& \sum_{g}^{\prime} S(0,1) \subset\left(\sum_{h}^{\prime}, \phi\right),
\end{aligned}
$$

and

$$
\left|h_{i}-g_{i}\right|<\psi, \quad i=1, \ldots, N \text {, }
$$

where

$$
d<\frac{1}{360 N^{2}}
$$

and

$$
\phi<\frac{1}{360 N^{2}}
$$

For $i \leqslant m$ define $h_{i}^{*}$ to be the projection of $h_{i}$ on $\sum_{h}^{\prime}$, and $h_{i}^{\prime \prime}$ by the equations

$$
\begin{aligned}
& h_{1}^{\prime \prime}=h_{1}^{*} \\
& h_{r}^{\prime \prime}=h_{r}^{*}-\sum_{t=1}^{r-1}\left(h_{t}^{\prime \prime} \cdot h_{r}^{*}\right) h_{t}^{\prime \prime} /\left|h_{t}^{\prime \prime}\right|^{2}, \quad r \leqslant m
\end{aligned}
$$

and then define $h_{i}^{\prime}$ to be the unit vector parallel to $h_{i}^{\prime \prime}$.

Let $\Pi_{h}, \Pi_{g}, \Pi_{h}^{\prime}, \Pi_{g}^{\prime}$ be the $N-m$ planes orthogonal to $\Sigma_{h}, \sum_{g}, \sum_{h}^{\prime}$ and $\sum_{g}^{\prime}$ respectively and for $i>m$ define $h_{i}^{*}$ to be the projection of $h_{i}$ onto $\Pi_{h}^{\prime}$, and $h_{i}^{\prime \prime}$ by the equations

$$
\begin{aligned}
h_{m+1}^{\prime \prime} & =h_{m+1}^{*} \\
h_{r}^{\prime \prime} & =h_{r}^{*}-\sum_{t=m+1}^{r-1}\left(h_{t}^{\prime \prime} \cdot h_{r}^{*}\right) h_{t}^{\prime \prime} /\left|h_{t}^{\prime \prime}\right|^{2}, \quad m<r \leqslant N
\end{aligned}
$$

and then define $h_{i}^{\prime}$ to be the unit vector parallel to $h_{i}^{\prime \prime}$.

Define $g_{i}^{\prime}(i=1, \ldots, N)$ similarly.

Then $h_{1}^{\prime}, \ldots, h_{N}^{\prime}$ and $g_{1}^{\prime}, \ldots, g_{N}^{\prime}$ will form two sets of orthogonal unit vectors such that $\Sigma_{h}^{\prime}$ and $\sum_{g}^{\prime}$ contain $h_{1}^{\prime}, \ldots, h_{m}^{\prime}$ and $g_{1}^{\prime}, \ldots, g_{m}^{\prime}$ respectively and

$$
\left|h_{i}^{\prime}-g_{i}^{\prime}\right|<4000 N^{3}(\phi+\psi) \text {. }
$$

Consider first $i \leqslant m$. By their definition the $h_{i}^{\prime \prime}$ will lie in $\Sigma_{h}^{\prime}$ and we can prove by induction that 


$$
h_{r}^{\prime \prime} \cdot h_{t}^{\prime \prime}=0, \quad t<r \leqslant m .
$$

It follows from (4) that $\sum_{h} S(0,1) \subset\left(\sum_{h}^{\prime}, d\right)$

and hence

$$
\left|h_{i}^{*}-h_{i}\right| \leqslant d
$$

so that

$$
\left|h_{i}^{*} \cdot h_{j}^{*}\right| \leqslant 2 d, \quad i \neq j .
$$

We will now prove by induction that if $q>p$

$$
\left|h_{p}^{\prime \prime} \cdot h_{q}^{*}\right|<3 d \text {. }
$$

and

$$
\left|h_{p}^{\prime \prime}\right|>\frac{1}{2} \text {. }
$$

If $p=1$ this is true by $(10),(17)$ and (16). Suppose then that (18), (19) hold for $p<p_{0}$. By (11)

$$
\begin{aligned}
\left|h_{p_{q}}^{\prime \prime} \cdot h_{q}^{*}\right| & \leqslant\left|h_{p_{0}}^{*} \cdot h_{q}^{*}\right|+4\left|\sum_{t=1}^{p_{0}-1}\left(h_{t}^{\prime \prime} \cdot h_{p_{0}}^{*}\right)\left(h_{t}^{\prime \prime} \cdot h_{q}^{*}\right)\right| \\
& \leqslant 2 d+4\left(p_{0}-1\right) \cdot 9 d^{2}
\end{aligned}
$$

which by (8)

$$
\leqslant 2 d+d \leqslant 3 d \text {. }
$$

By (11), (18) and (19)

$$
\left|h_{p_{0}}^{\prime \prime}-h_{p_{0}}^{*}\right| \leqslant 6 N d \text {. }
$$

Thus by (16)

$$
\left|h_{p_{0}}^{\prime \prime}-h_{p_{0}}\right| \leqslant(6 N+1) d \text {. }
$$

Hence $\left|h_{p_{\mathrm{e}}}^{\prime \prime}\right|>\frac{1}{2}$ which completes the induction. Moreover, again by (20),

$$
\left|h_{i}-h_{i}^{\prime}\right| \leqslant 2 \sin \frac{1}{2} \sin ^{-1}(6 N+1) d \leqslant(12 N+2) d, \quad i \leqslant m .
$$

Now by Lemma 1 and (4) $\quad \Pi_{h}^{\prime} S(0,1) \subset\left(\Pi_{h}, 2 d\right)$

and so repeating the above procedure we obtain for $i>m$

$$
\left|h_{i}-h_{i}^{\prime}\right| \leqslant(24 N+4) d \text {. }
$$

The $h_{i}^{\prime}$ are unit vectors by definition, and by (15) and the fact that $\Pi_{h}^{\prime}$ and $\sum_{h}^{\prime}$ are orthogonal, they will be orthogonal.. Moreover by induction from their definition we can prove that the first $m$ of them lie in $\Sigma_{h}^{\prime}$. We may similarly prove the corresponding result for the $g_{i}^{\prime}$.

Moreover using (6) instead of (4) we may derive a set of $N$ orthogonal unit vectors $\left\{f_{i}\right\}$ from the $h_{i}^{\prime}$, just as the $h_{i}^{\prime}$ were derived from the $h_{i}$. We will then have as an analogue of (23)

$$
\left|f_{i}-h_{i}^{\prime}\right| \leqslant(24 N+4) \phi
$$

and $f_{1} \ldots f_{m}$ will lie in $\sum_{g}^{\prime}$. 
Let $g_{i}^{* *}, i \leqslant m$, be the projection of $g_{i}$ into $\sum_{h}^{\prime}$. Then we have

$$
\begin{gathered}
g_{i}^{*}=\sum_{r=1}^{m}\left(g_{i} \cdot f_{r}\right) f_{r}, \quad i \leqslant m \\
g_{i}^{* *}=\sum_{r=1}^{m}\left(g_{i} \cdot h_{r}^{\prime}\right) h_{r}^{\prime}, \quad i \leqslant m .
\end{gathered}
$$

and

Hence by (24)

$$
\left|g_{i}^{*}-g_{i}^{* *}\right| \leqslant \sum_{r=1}^{m}\left|g_{i} \cdot f_{r}\right|\left|f_{r}-h_{r}^{\prime}\right|+\left|h_{r}^{\prime}\right|\left|g_{i} \cdot f_{r}-g_{i} \cdot h_{r}^{\prime}\right| \leqslant\left(24 N^{2}+8 N\right) \phi .
$$

Now by (7) $\left|h_{i}^{*}-g_{i}^{* *}\right| \leqslant \psi$ and hence by (27)

$$
\left|h_{i}^{*}-g_{i}^{*}\right| \leqslant \psi+\left(48 N^{2}+8 N\right) \phi
$$

I will now prove by induction that if $r>t$

$$
\left|h_{t}^{\prime \prime} \cdot h_{r}^{*}-g_{t}^{\prime \prime} \cdot g_{r}^{*}\right|<3 \psi+3\left(48 N^{2}+8 N\right) \phi
$$

and

$$
\left|h_{i}^{\prime \prime}-g_{i}^{\prime \prime}\right| \leqslant 8 N \psi+8 N\left(48 N^{2}+8 N\right) \phi .
$$

These are true for $t=1$ by (28), (10). Suppose they hold for $r>t$ and $t<t_{0}$. By (11) and (18) and the corresponding results for the $g_{i}$

$$
\begin{aligned}
\left|h_{t_{0}}^{\prime \prime} \cdot h_{r}^{*}-g_{t_{0}}^{\prime \prime} \cdot g_{r}^{*}\right| \leqslant & \left|h_{t_{0}}^{*} \cdot h_{r}^{*}-g_{t_{0}}^{*} \cdot h_{r}^{*}\right|+\left|g_{t_{0}}^{*} \cdot h_{r}^{*}-g_{t_{0}}^{*} \cdot g_{r}^{*}\right| \\
& +\left.\left|\sum_{t=1}^{t_{0}-1}\left(h_{t}^{\prime \prime} \cdot h_{t_{0}}^{*}\right)\left(h_{t}^{t^{\prime}} \cdot h_{r}^{*}\right) /\right| h_{t}^{\prime \prime}\right|^{2}-\left(g_{t}^{\prime \prime} \cdot g_{t_{0}}^{*}\right)\left(g_{t}^{\prime \prime} \cdot g_{r}^{*}\right) /\left|g_{t}^{\prime \prime}\right|^{2} \\
\leqslant & \left|h_{t_{0}}^{*}-g_{t_{v}}^{*}\right|+\left|h_{r}^{*}-g_{r}^{*}\right|+\sum_{t=1}^{t_{0}-1}\left|h_{t}^{\prime \prime} \cdot h_{t_{0}}^{*}\right|\left|h_{t}^{\prime \prime} \cdot h_{r}^{*}-g_{t}^{\prime \prime} \cdot g_{r}^{*}\right| /\left|h_{t}^{\prime \prime}\right|^{2} \\
& +\sum_{t=1}^{t_{0}-1}\left|g_{t}^{\prime \prime} \cdot g_{r}^{*}\right|\left|h_{t}^{\prime \prime} \cdot h_{t_{\mathrm{o}}}^{*}-g_{t}^{\prime \prime} \cdot g_{t_{\mathrm{v}}}^{*}\right| /\left|h_{t}^{\prime \prime}\right|^{2} \\
& +\left.\sum_{t=1}^{t_{\mathrm{v}}-1}\left|g_{t}^{\prime \prime} \cdot g_{t_{\mathrm{o}}}^{*}\right|\left|g_{t}^{\prime \prime} \cdot g_{r}^{*}\right||1 /| h_{t}^{\prime \prime}\right|^{2}-1 /\left|g_{t}^{\prime \prime}\right|^{2} \mid \\
\leqslant & 2 \psi+2\left(48 N^{2}+8 N\right) \phi+(12 N d+12 N d)\left(3 \psi+3\left(48 N^{2}+8 N\right) \phi\right), \\
& +9 N d^{2} \cdot 16\left(8 N \psi+8 N\left(48 N^{2}+8 N\right) \phi\right) \\
\leqslant & 3 \psi+3\left(44 N^{2}+8 N\right) \phi .
\end{aligned}
$$

Furthermore by (28), (29), (30), (18) and (19) 


$$
\begin{aligned}
\left|h_{t_{0}}^{\prime \prime}-g_{t_{0}}^{\prime \prime}\right| \leqslant \mid & \left|h_{t_{0}}^{*}-g_{t_{0}}^{*}\right|+\left.\sum_{t=1}^{t_{0}-1}\left|\left(h_{t}^{\prime \prime} \cdot h_{t_{0}}^{*}\right) h_{t}^{\prime \prime} /\right| h_{t}^{\prime \prime}\right|^{2}-\left(g_{t}^{\prime \prime} \cdot g_{t_{0}}^{*}\right) g_{t}^{\prime \prime} /\left|g_{t}^{\prime \prime}\right|^{2} \mid \\
\leqslant & \left|h_{t_{0}}^{*}-g_{t_{0}}^{*}\right|+\sum_{t=1}^{t_{0}-1}\left|h_{t}^{\prime \prime} \cdot h_{t_{0}}^{*}-g_{t}^{\prime \prime} \cdot g_{t_{0}}^{*}\right| /\left|h_{t}^{\prime \prime}\right|+\left.\left|g_{t}^{\prime \prime} \cdot g_{t_{0}}^{*}\right|\left|h_{t}^{\prime \prime} /\right| h_{t}^{\prime \prime}\right|^{2}-g_{t}^{\prime \prime} /\left|g_{t}^{\prime \prime}\right|^{2} \mid \\
\leqslant & +\left(48 N^{2}+8 N\right) \phi+2 N\left(3 \psi+3\left(48 N^{2}+8 N\right) \phi\right) \\
& +3 N d \cdot 12\left(8 N \psi+8 N\left(48 N^{2}+8 N\right) \phi\right) . \\
\leqslant & 8 N \psi+8 N\left(48 N^{2}+8 N\right) \phi-
\end{aligned}
$$

Hence, since by (19) $\left|h_{i}^{\prime \prime}\right|>\frac{1}{2}$ and similarily $\left|g_{i}^{\prime \prime}\right|>\frac{1}{2}$, we have for $i \leqslant m$

$$
\begin{aligned}
\left|h_{i}^{\prime}-g_{i}^{\prime}\right| & \leqslant 2 \sin \frac{1}{2} \sin ^{-1}\left(16 N \psi+16 N\left(48 N^{2}+8 N\right) \phi\right) \\
& \leqslant 32 N \psi+32 N\left(48 N^{2}+8 N\right) \phi .
\end{aligned}
$$

Now by Lemma 1 and (4), (5), (6)

$$
\begin{aligned}
& \Pi_{h}^{\prime} S(0,1) \subset\left(\Pi_{h}, 2 d\right) \\
& \Pi_{g}^{\prime} S(0,1) \subset\left(\Pi_{g}, 2 d\right) \\
& \Pi_{g}^{\prime} S(0,1) \subset\left(\Pi_{h}^{\prime}, 2 \phi\right) .
\end{aligned}
$$

Hence we may repeat the above arguments for $i>m$ to prove that

$$
\left|h_{i}^{\prime}-g_{i}^{\prime}\right| \leqslant 16 N \psi+32 N\left(24 N^{2}+8 N\right) \phi .
$$

(31) and (35) imply (14) since $N>2$, which proves the lemma.

\section{LEMMA 4 .}

$$
\text { If } \varepsilon \leqslant 2^{-2000 N^{2}} \text { and } N \geqslant 3
$$

$$
\text { and } \quad \begin{aligned}
\lambda^{9} & =2^{2 N+20} N^{12 N_{\varepsilon}-\frac{1}{3}} \\
\xi & =\varepsilon^{\frac{1}{b}} \\
R_{0} & =1 \\
\varrho & =2^{23 N} \varepsilon^{1 / 19} \\
\zeta & =2^{50 N} \varepsilon^{1 /(12 N+24)}
\end{aligned}
$$$$
\text { (37) } \quad \eta=N^{-24 N} \varepsilon^{\frac{2}{3}}
$$$$
\text { (39) } \quad \alpha_{1}=\varepsilon^{\frac{1}{2}}
$$$$
\text { (4I) } \quad \mu=\varepsilon^{-\frac{1}{2}}
$$$$
\text { (43) } \delta=\frac{1}{2 N} \varepsilon^{\frac{2}{3}}
$$$$
\text { (45) } \theta=\frac{\pi}{4} \text {, }
$$

then $\eta=2^{4 N+40} \lambda^{-18}$

(47) $\lambda>8$

$$
\begin{array}{rlrl}
\eta & <\left\{120 N(N+20) 2^{6 N+41}(8 N)^{N}\right\}^{-1}(49) & \xi \alpha_{1}>2 \varepsilon R_{0}+2 \varepsilon \mu \alpha_{1} \\
\eta & \geqslant 4000 N^{3}(4 \varepsilon+\delta \lambda \varrho) & (51) & \lambda+2 \varepsilon \mu<\mu \\
\delta \lambda \varrho & <\frac{1}{360 N^{2}} & (53) & 4 \varepsilon<\frac{1}{360 N^{2}}
\end{array}
$$




$$
\begin{aligned}
& \delta \geqslant 10 N(N+20) 2^{6 N+41}(8 N)^{N} \eta \\
& \text { (55) } \quad \xi \geqslant 4 \varepsilon \mu+N \delta \mu \\
& \alpha_{1} \varrho \geqslant 400 \varepsilon R_{0} \\
& \text { (57) } \quad \zeta \geqslant 3200 N^{2} 2^{36 N}\left(\delta \xi^{-\frac{1}{4}}+\xi^{\frac{1}{2}}+\xi^{1 /(4 N+8)}\right) \\
& \sin \theta \geqslant\left\{\frac{2 N \delta}{100}+\frac{2 \xi}{\mu \varrho}+\sin \frac{1}{2} \theta\right\} \\
& \text { (59) } \quad N \zeta+2^{18 N+3} N^{3} \delta \xi^{\frac{1}{3}} \leqslant \frac{1}{20 \overline{0}} \\
& 6 \lambda \delta \leqslant \frac{1}{20} \\
& \text { (61) } \quad \frac{1}{100}+\xi+8 N \delta+2^{18 N+3} N \xi^{\sharp}<\frac{1}{50} \\
& 2^{18 N+3} N \xi^{\frac{1}{4}} \varrho^{-1} \leqslant \frac{1}{200} \\
& \text { (63) } \quad(4 N \delta+\xi) \varrho^{-1} \leqslant \frac{1}{200} \\
& \varrho<\frac{1}{10 \overline{0}} \\
& \text { (65) } \quad N \delta \mu \leqslant \frac{1}{2} \xi \text {. }
\end{aligned}
$$

When any of the above symbols occur in the subsequent work they will be taken to have the values here assigned.

LEMMA 5. Suppose $\left\{X_{i}\right\}$ is a finite set of points and $f\left(X_{i}\right)$ a unit vector defined at these points such that

$$
\left|X_{i}-X_{i}\right|>\alpha . \quad i \neq i^{\prime}
$$

and

$$
\left|f\left(X_{i}\right)-f\left(X_{i}\right)\right|<\eta \quad \text { provided }\left|X_{i}^{--} X_{i}\right|<\lambda \alpha
$$

Then for all $X$ such that

$$
\left|X-X_{i}\right| \leqslant 2 \propto \quad \text { for some } i
$$

we may define $f(X)$ so that

and

$$
\begin{gathered}
1+2 \eta \geqslant|f(X)| \geqslant 1-2 \eta \\
f(X)=f\left(X_{i}\right) \quad \text { when } X=X_{i}
\end{gathered}
$$

while if each point of the interval $X^{\prime} X^{\prime \prime}$ lies within $2 \alpha$ of some $X_{i}$ then

$$
\left|f\left(X^{\prime}\right)-f\left(X^{\prime \prime}\right)\right|<10(N+20) 2^{6 N+41} \eta \frac{\left|X^{\prime}-X^{\prime \prime}\right|}{\alpha} .
$$

Define $f(X)$ by

$$
f(X)=\frac{\sum_{i} f\left(X_{i}\right) /\left|X-X_{i}\right|^{(N+20)}}{\sum_{i}\left|X-X_{i}\right|^{-(N+20)}} \quad \text { when } X \neq X_{i} \text { for any } i
$$

Let $X_{0}$ be the nearest $X_{i}$ to $X$; that is, choose $X_{0}$ to be an $X_{i}$ so that $\left|X-X_{0}\right|=$ $=\min _{i}\left|X-X_{i}\right|$. Then by (67)

$$
\left|X-X_{i}\right|>\frac{1}{2} \alpha, \quad i \neq 0
$$

and by (69)

$$
\left|X-X_{0}\right| \leqslant 2 \alpha \text {. }
$$


Now there are, by $(67)$, at most $2^{N}(r+2)^{N}$ points $X_{i}$ such that

Hence by $(75)$

$$
r \alpha \leqslant\left|X_{0}-X_{i}\right| \leqslant(r+1) \alpha .
$$

$$
\begin{aligned}
& \sum_{\left|X_{0}-X_{i}\right| \geqslant \lambda^{\prime} \alpha}\left[\frac{\left|X-X_{0}\right|}{\left|X-X_{i}\right|}\right]^{N+19} \leqslant \sum_{r>\lambda^{\prime}-1} 2^{N}(r+2)^{N}\left[\frac{2}{r-2}\right]^{N+19} \\
& \leqslant 2^{2 N+19} \frac{\left(\lambda^{\prime}+1\right)^{N}}{\left(\lambda^{\prime}-3\right)^{N+17}} \sum_{r>\lambda^{\prime}-1} \frac{1}{(r-2)(r-3)} \leqslant 2^{2 N+19} \frac{\left(\lambda^{\prime}+1\right)^{N}}{\left(\lambda^{\prime}-4\right)\left(\lambda^{\prime}-3\right)^{N+17}} .
\end{aligned}
$$

Hence

$$
\left|f(X)-f\left(X_{0}\right)\right| \leqslant \frac{\sum_{i}\left|f\left(X_{i}\right)-f\left(X_{0}\right)\right|\left|\frac{X-X_{0}}{X-X_{i}}\right|^{N+20}}{\sum_{i}\left|\frac{X-X_{0}}{X-X_{i}}\right|^{N+20}}
$$

which by $(68)$

$$
\leqslant \eta+\sum_{\left|X_{0}-X_{i}\right| \geqslant \lambda x} 2\left|\frac{X-X_{0}}{X-X_{i}}\right|^{N+20}
$$

which by (77) and (74), (75)

$$
\leqslant \eta+2^{2 N+22} \frac{(\lambda+1)^{N}}{(\lambda-4)(\lambda-3)^{N+17}}
$$

which by $(48)$

$$
\leqslant \eta+2^{3 N+40} \lambda^{-18}
$$

and by (47)

$\leqslant 2 \eta$.

\section{Moreover (1)}

$$
\begin{aligned}
& \left|\frac{\partial}{\partial X} f(X)\right|=\left|\frac{\partial}{\partial X}\left[f(X)-f\left(X_{0}\right)\right]\right|=\left|\frac{\partial}{\partial X} \frac{\sum_{i}\left[f\left(X_{i}\right)-f\left(X_{0}\right)\right]\left|\frac{X-X_{0}}{X-X_{i}}\right|^{N+20}}{\sum_{i}\left|\frac{X-X_{0}}{X-X_{i}}\right|^{N+20}}\right| \\
& \leqslant \frac{(N+20) \sum_{i \neq 0}\left|f\left(X_{i}\right)-f\left(X_{0}\right)\right|\left|\frac{X-X_{0}}{X-X_{i}}\right|^{N+19}|| \frac{X-X_{i}\left|\frac{\partial}{\partial X}\right| X-X_{0}|-| X-X_{0}\left|\frac{\partial}{\partial X}\right| X-X_{i} \mid}{\left|X-X_{i}\right|^{2}} \mid}{\sum_{i}\left|\frac{X-X_{0}}{X-X_{i}}\right|^{N+20}} \mid
\end{aligned}
$$$$
+(N+20) \frac{\left|f(X)-f\left(X_{0}\right)\right|}{\sum_{i}\left|\frac{X-X_{0}}{X-X_{i}}\right|^{N+20}} \sum_{i \neq 0}\left|\frac{X-X_{0}}{X-X_{i}}\right|^{N+19}\left|\frac{\left|X-X_{i}\right| \frac{\partial}{\partial X}\left|X-X_{0}\right|-\left|X-X_{0}\right| \frac{\partial}{\partial X}\left|X-X_{i}\right|}{\left|X-X_{i}\right|^{2}}\right| .
$$

Now

$$
\frac{\partial}{\partial X}\left|X-X_{i}\right| \leqslant 1 \quad \text { and } \quad \sum_{i}\left|\frac{X-X_{0}}{X-X_{i}}\right|^{N+20} \geqslant 1
$$

(1) Differentiation along an arbitrary line. 
so that by $(78),(74),(75)$ the above is

$$
\leqslant \frac{10(N+20)}{\alpha}\left|\sum_{i \neq 0}\right| f\left(X_{i}\right)-\left.f\left(X_{0}\right)|| \frac{X-X_{0}}{X-X_{i}}\right|^{N+19}+2 \eta \sum_{i \neq 0}\left|\frac{X-X_{0}}{X-X_{i}}\right|^{N+19} \mid
$$

which by (68), (74), (75) and (77) with $\lambda^{\prime}=\lambda$ and $\lambda^{\prime}=7$

$$
\begin{aligned}
& \leqslant\left.\frac{10(N+20)}{\alpha}\left|3 \eta \sum_{i \neq 0}\right| \frac{X-X_{0}}{X-X_{i}}\right|^{N+19}+2 \sum_{\left|X_{0}-X_{i}\right| \geqslant \lambda \alpha}\left|\frac{X-X_{0}}{X-X_{i}}\right|^{N+19} \mid \\
& \leqslant \frac{10(N+20)}{\alpha}\left|3 \eta\left(2^{N} \cdot 8^{N} \cdot 4^{N+19}+\frac{2^{2 N+19} 8^{N}}{3 \cdot 4^{N+17}}\right)+\frac{2 \cdot 2^{2 N+19}(\lambda+1)^{N}}{(\lambda-4)(\lambda-3)^{N+17}}\right| \\
& \leqslant \frac{10(N+20)}{\alpha}\left|2^{6+40} \eta+2^{3 N+38} \lambda^{-18}\right|,
\end{aligned}
$$

which by (47)

$$
\leqslant \frac{10(N+20)}{\alpha} \cdot 2^{6 N+41} \eta
$$

Now (78) and (79) imply (70) and (72) respectively so that the lemma follows.

LEMMA 6. Suppose ue have $t$ orthogonal unit vectors $f_{1}\left(X_{i}\right), \ldots, f_{t}\left(X_{i}\right)$ defined at the points $\left\{X_{i}\right\}$ of Lemma 5, satisfying the conditions of that lemma, and extended as in that lemma. Then there will exist a set of $t$ orthogonal unit vectors $g_{1}(X), \ldots, g_{t}(X)$, coinciding with the $\left\{f_{t}(X)\right\}$ at the $\left\{X_{i}\right\}$, defined at all $X$ such that

$$
\left|X-X_{i}\right| \leqslant 2 \alpha \quad \text { for some } i
$$

and such that, if each point of the interval $X^{\prime} X^{\prime \prime}$ lies within $2 \alpha$ of some $X_{i}$, then for $1 \leqslant t^{\prime} \leqslant t$

$$
\left|g_{t^{\prime}}\left(X^{\prime}\right)-g_{t^{\prime}}\left(X^{\prime \prime}\right)\right|<10 N(N+20) 2^{6 N+41}(8 N)^{t^{\prime}} \eta \frac{\left|X^{\prime}-X^{\prime \prime}\right|}{\alpha}
$$

Define $g_{1}(X)$ to be the unit vector parallel to $f_{1}(X)$ and define $g_{t^{\prime}}(X), t^{\prime}>1$, inductively as the unit vector parallel to $g_{t}^{*}(X)$, where

$$
g_{t^{\prime}}^{*}(X)=f_{t^{\prime}}(X)-\sum_{r^{\prime}=1}^{t^{\prime}-1}\left\{f_{t^{\prime}}(X) \cdot g_{r}(X)\right\} g_{r}(X)
$$

Clearly the $g_{t^{\prime}}(X)$ will be orthogonal. 
I will now prove (81) by induction. For if $t^{\prime}=1$ then by (70) and (72), since $\eta<\frac{1}{4}$.

$$
\begin{aligned}
\left|g_{1}\left(X^{\prime}\right)-g_{1}\left(X^{\prime \prime}\right)\right| & \leqslant 2 \sin \frac{1}{2} \sin ^{-1} \frac{10(N+20) 2^{6 N+41} \eta\left|X^{\prime}-X^{\prime \prime}\right|}{\alpha(1-2 \eta)} \\
& \leqslant 40(N+20) 2^{6 N+41} \eta \frac{\left|X^{\prime}-X^{\prime \prime}\right|}{\alpha}
\end{aligned}
$$

which implies (81) for $t^{\prime}=1$.

Suppose now that (81) holds for $t^{\prime}<t_{0}$. Then since $g_{r}(X)$ is a unit vector

$$
\begin{aligned}
& \left|g_{t_{0}}^{*}\left(X^{\prime}\right)-g_{t_{0}}^{*}\left(X^{\prime \prime}\right)\right| \leqslant \mid f_{t_{0}}\left(X^{\prime}\right)-f_{t_{0}}\left(X^{\prime \prime}\left|+\sum_{r=1}^{t_{0}-1}\right| f_{t_{0}}\left(X^{\prime}\right) \cdot g_{r}\left(X^{\prime}\right)-f_{t_{0}}\left(X^{\prime \prime}\right) \cdot g_{r}\left(X^{\prime \prime}\right) \mid\right. \\
& +\sum_{r=1}^{t_{0}-1}\left|f_{t_{0}}\left(X^{\prime \prime}\right) \cdot g_{r}\left(X^{\prime \prime}\right)\right|\left|g_{r}\left(X^{\prime}\right)-g_{r}\left(X^{\prime \prime}\right)\right| \\
& \leqslant\left|f_{t_{0}}\left(X^{\prime}\right)-f_{t_{0}}\left(X^{\prime \prime}\right)\right|+\sum_{r=1}^{t_{0}-1}\left|f_{t_{0}}\left(X^{\prime}\right)-f_{t_{0}}\left(X^{\prime \prime}\right)\right|\left|g_{r}\left(X^{\prime}\right)\right| \\
& +\left|f_{t_{0}}\left(X^{\prime \prime}\right)\right|\left|g_{r}\left(X^{\prime}\right)-g_{r}\left(X^{\prime \prime}\right)\right|+\left|f_{t_{0}}\left(X^{\prime \prime}\right)\right|\left|g_{r}\left(X^{\prime}\right)-g_{r}\left(X^{\prime \prime}\right)\right| \\
& \leqslant\left|f_{t_{0}}\left(X^{\prime}\right)-f_{t_{0}}\left(X^{\prime \prime}\right)\right|+\sum_{r=1}^{t_{0}-1}\left|f_{t_{0}}\left(X^{\prime}\right)-f_{t_{0}}\left(X^{\prime \prime}\right)\right|+2\left|g_{r}\left(X^{\prime}\right)-g_{r}\left(X^{\prime \prime}\right)\right|\left|f_{t_{0}}\left(X^{\prime \prime}\right)\right|,
\end{aligned}
$$

which by (70), (72) and (81)

$$
\leqslant 10 N(N+20) 2^{6 N+41} \eta \frac{\left|X^{\prime}-X^{\prime \prime}\right|}{\alpha}+2 N(1+2 \eta) \cdot(8 N)^{t_{\mathrm{a}-1}} 10 N(N+20) 2^{6 N+40} \eta \frac{\left|X^{\prime}-X^{\prime \prime}\right|}{\alpha}
$$

which since $\eta<\frac{1}{4}$ and $t_{0}>1$

$$
\leqslant 10 N(N+20) 2^{6 N+41} \cdot(8 N)^{t_{0}} \eta \frac{\left|X^{\prime}-X^{\prime \prime}\right|}{\alpha} \cdot \frac{1+3 N}{8 N}
$$

In particular taking $X^{\prime \prime}$ to be an $X_{i}$ such that $\left|X^{\prime}-X^{\prime \prime}\right| \leqslant 2 \alpha$, and noting that, by induction on (82), we can show that $g_{t_{0}}^{*}\left(X_{i}\right)=f_{t_{0}}\left(X_{r}\right)$ which is a unit vector, we obtain, using (49) that

$$
\left|g_{t_{0}}^{*}\left(X^{\prime}\right)\right| \geqslant 1-20 N(N+20) 2^{6 N+41}(8 N)^{N} \eta \geqslant \frac{5}{6}
$$

and similarly that

$$
\left|g_{t_{0}}^{*}\left(X^{\prime \prime}\right)\right| \geqslant \frac{5}{6} \text {. }
$$

4-60173032. Acta mathematica. 104. Imprimé le 23 septembre 1960 
Henc by (84)

$$
\begin{aligned}
\left|g_{t_{0}}\left(X^{\prime}\right)-g_{t_{0}}\left(X^{\prime \prime}\right)\right| & \leqslant 2 \sin \frac{1}{2} \sin ^{-1}\left[10 N(N+20) 2^{6 N+41}(8 N)^{t_{t}} \eta \frac{\left|X^{\prime}-X^{\prime \prime}\right|}{\alpha} \cdot \frac{1+3 N}{8 N} \cdot \frac{6}{5}\right] \\
& \leqslant 10 N(N+20) 2^{6 N+41}(8 N)^{t_{0}} \eta \frac{\left|X^{\prime}-X^{\prime \prime}\right|}{\alpha} \cdot \frac{1+3 N}{4 N} \cdot \frac{6}{5} \\
& \leqslant 10 N(N+20) 2^{6 N+41}(8 N)^{t_{0}} \eta \frac{\left|X^{\prime}-X^{\prime \prime}\right|}{\alpha}
\end{aligned}
$$

which establishes the lemma.

LемMA 7. Suppose $S_{0}$ is a set and $S$ is a subset of $S_{0}$ such that if $X \in S$ and $0<R<R_{0}$ then there will exist an m-plane ${ }_{R} \Sigma_{X}$ through $X$ such that

$$
S_{0} S(X, R) \subset\left({ }_{R} \Sigma_{X}, \varepsilon R\right) S(X, R)
$$

and

$$
{ }_{R} \Sigma_{X} S(X, R) \subset\left(S_{0}, \varepsilon R\right) S(X, R)
$$

and suppose further that there exists a plane $\Sigma$ such that

$$
S_{0} \subset\left(\Sigma, \varepsilon R_{0}\right) \text {. }
$$

Then if we have a sequence of numbers $\left\{\alpha_{j}\right\}$ such that

$$
\frac{\alpha_{j+1}}{\alpha_{j}}=\varrho
$$

and a set of points $\left\{X_{i j}\right\}$ belonging to $S$ such that

$$
\left|X_{i j}-X_{i^{\prime} j}\right|>\alpha_{j} \quad i \neq i^{\prime}
$$

and for all $X \in S$

$$
\operatorname{Min}_{i}\left|X-X_{i j}\right| \leqslant \alpha_{j}
$$

then there will exist, for each $j$, and $X \in\left(S, \alpha_{j}\right)$ a set of $N$ orthogonal unit vectors

$$
\left\{h_{j k}(X)\right\} \quad k=1, \ldots, N
$$

such that if the interval $X^{\prime} X^{\prime \prime}$ lies within $\alpha_{j}$ of $S$ then

$$
\left|h_{j k}\left(X^{\prime}\right)-h_{j k}\left(X^{\prime \prime}\right)\right| \leqslant \frac{\delta}{\alpha_{j}}\left|X^{\prime}-X^{\prime \prime}\right|
$$

and if $X \in S$ then

$$
\begin{array}{ll}
\text { and if } X \in S \text { then } & S_{0} S\left(X, \mu \alpha_{j}\right) \subset\left(\sum_{X}^{j}, \xi \alpha_{j}\right) S\left(X, \mu \alpha_{j}\right) \\
\text { and } & \sum_{X}^{j} S\left(X, \mu \alpha_{j}\right) \subset\left(S_{0}, \xi \alpha_{j}\right) S\left(X, \mu \alpha_{j}\right),
\end{array}
$$

where $\Sigma_{X}^{j}$ is the m-plane through $X$ containing the vectors $\left\{h_{j k}(X)\right\} k=1, \ldots, m$. 
Consider first the case $j=1$. Let $\left\{h_{1 k}(X)\right\}$ be a system of orthogonal unit vectors independent of $X$ such that the first $m$ of them are contained in $\Sigma$. $\Sigma_{X}^{1}$ will be the plane through $X$ parallel to $\Sigma$. (9I) will be satisfied since $\left|h_{1 k}\left(X^{\prime}\right)-h_{1 k}\left(X^{\prime \prime}\right)\right|=0$. If $X \in S$ then by (87) $X \subset\left(\Sigma, \varepsilon R_{0}\right)$ and hence by (87) and (50) $S_{0} S\left(X, \mu \alpha_{1}\right) \subset$ $\subset\left(\sum \varepsilon, R_{0}\right) S\left(X, \mu \alpha_{1}\right) \subset\left(\Sigma_{X}^{1}, 2 \varepsilon R_{0}\right) S\left(X, \mu \alpha_{1}\right) \subset\left(\Sigma_{X}^{1}, \xi \alpha_{1}\right) S\left(X, \mu \alpha_{1}\right)$, which proves $(92)$; and using (86)

$$
\begin{aligned}
{ }_{\mu \alpha_{1}} \Sigma_{X} S\left(X, \mu \alpha_{1}\right) \subset\left(S_{0}, \varepsilon \mu \alpha_{1}\right) S\left(X, \mu \alpha_{1}\right) \subset(\Sigma, \varepsilon & \left.R_{0}+\varepsilon \mu \alpha_{1}\right) S\left(X, \mu \alpha_{1}\right) \subset \\
& \left(\sum_{X}^{1}, 2 \varepsilon R_{0}+\varepsilon \mu \alpha_{1}\right) S\left(X, \mu \alpha_{1}\right) .
\end{aligned}
$$

Hence

$$
\Sigma_{X}^{1} S\left(X, \mu \alpha_{1}\right) \subset\left({ }_{\mu \alpha_{1}} \Sigma_{X}, 2 \varepsilon R_{0}+\varepsilon \mu \alpha_{1}\right) S\left(X, \mu \alpha_{1}\right)
$$

which by (86)

$$
\subset\left(S_{0}, 2 \varepsilon R_{0}+2 \varepsilon \mu \alpha_{1}\right) S\left(X, \mu \alpha_{1}\right)
$$

which by (50)

$$
\subset\left(S_{0}, \xi \alpha_{1}\right) S\left(X, \mu \alpha_{1}\right)
$$

which proves (93).

We will now prove the lemma by induction on $j$. Suppose then that the $\left\{h_{j k}(X)\right\}$ exist as demanded. Since $2 \mu \alpha_{1}<R_{0}$, we may by (85) and (86) define $\Sigma_{X_{i j+1}+1}^{j+1}$ to be a plane through $X_{i j+1}$ such that

$$
S_{\mathbf{0}} S\left(X_{i j+1}, 2 \mu \alpha_{j+1}\right) \subset\left(\sum_{X_{i j+1}}^{j+1}, 2 \varepsilon \mu \alpha_{j+1}\right) S\left(X_{i j+1}, 2 \mu \alpha_{j+1}\right)
$$

and

$$
\sum_{i j+1}^{j+1} S\left(X_{i j+1}, 2 \mu \alpha_{j+1}\right) \subset\left(S_{0}, 2 \varepsilon \mu \alpha_{j+1}\right) S\left(X_{i j+1}, 2 \mu \alpha_{j+1}\right)
$$

I will now apply Lemma 3. Take $\left\{h_{j k}\left(X_{i j+1}\right)\right\}$ to be the $\left\{h_{k}\right\}$ of that lemma, define $\left\{h_{j+1, k}\left(X_{i j+1}\right)\right\}$ to be the $\left\{h_{k}^{\prime}\right\}$ of that lemma and similarly for the $g$ of that lemma taking some $i^{\prime} \neq i$.

The planes $\Sigma_{h}, \Sigma_{g}, \Sigma_{h}^{\prime}, \Sigma_{g}^{\prime}$ will be planes through $O$ parallel to $\Sigma_{X_{i j+1}}, \Sigma_{X_{i^{\prime} j+1}^{\prime}}$, $\Sigma_{X_{i j+1}^{j+1}}^{j+1}$ and $\Sigma_{X_{i^{\prime} j+1}^{j+1}}^{j+1}$ respectively.

Suppose

$$
\left|X_{i j+1}-X_{i^{\prime} j+1}\right|<\lambda \alpha_{j+1} .
$$

Then by our inductive hypothesis (91), since $\lambda \alpha_{j+1}=\lambda \varrho \alpha_{j}<\alpha_{j}$

$$
\left|h_{j k}\left(X_{i j+1}\right)-h_{j k}\left(X_{i^{\prime} j+1}\right)\right| \leqslant \delta \lambda \varrho
$$

which we will take as (7) of Lemma 3 with

Now by (95)

$$
\psi=\delta \lambda \varrho
$$




$$
\begin{aligned}
\sum_{X_{i j+1}}^{j+1} S\left(X_{i j+1}, \mu \alpha_{j+1}\right) & \subset\left(S_{0}, 2 \varepsilon \mu \alpha_{j+1}\right) S\left(X_{i j+1}, 2 \mu \alpha_{j+1}\right) \\
& \subset\left(S_{0} S\left(X_{i j+1}, 2 \mu \alpha_{j+1}+2 \varepsilon \mu \alpha_{j+1}\right), 2 \varepsilon \mu \alpha_{j+1}\right)
\end{aligned}
$$

which by $(92) \subset\left(\Sigma_{X_{i j+1}}^{j}, \xi \alpha_{j}+2 \varepsilon \mu \alpha_{j+1}\right)$, since $2 \mu \alpha_{j+1}+2 \varepsilon \mu \alpha_{j+1}<\mu \alpha_{j}$.

This together with the corresponding result for $i^{\prime}$ may be taken as (4) and (5) where

$$
d=\frac{\xi \alpha_{j}+2 \varepsilon \mu \alpha_{j+1}}{\mu \alpha_{j+1}}
$$

(8) will then be satisfied by the definition of $\xi, \mu$ and $\varrho$. By (95) for $i^{\prime}$

$$
\Sigma_{X_{i^{\prime} j+1}^{j+1}}^{j+1} S\left(X_{i^{\prime}, j+1}, \mu \alpha_{j+1}\right) \subset\left(S_{0}, 2 \varepsilon \mu \alpha_{j+1}\right) S\left(X_{i^{\prime}, j+1}, \mu \alpha_{j+1}\right),
$$

which by (96)

$$
\begin{aligned}
& \subset\left(S_{0}, 2 \varepsilon \mu \alpha_{j+1}\right) S\left(X_{i j+1},(\lambda+\mu) \alpha_{j+1}\right), \\
& \subset\left(S_{0} S\left(X_{i j+1},(\lambda+\mu+2 \varepsilon \mu) \alpha_{j+1}\right), 2 \varepsilon \mu \alpha_{j+1}\right),
\end{aligned}
$$

which by (52) and (94)

$$
\subset\left(\sum_{i j+1}^{j+1}, 4 \varepsilon \mu \alpha_{j+1}\right) \text {. }
$$

This may be taken as (6) of Lemma 3, where

$$
\dot{\phi}=4 \varepsilon,
$$

and (9) is satisfied by the definition of $\varepsilon$.

We may therefore define $\left\{h_{j+1}\left(X_{i j+1}\right)\right\}$ and by (14), (98), (100)

$$
\left|h_{j+1 k}\left(X_{i j+1}\right)-h_{j+1 k}\left(X_{i^{\prime} j+1}\right)\right|<1000 N^{3}(\delta \lambda \varrho+4 \varepsilon)
$$

which by (51)

$$
\leqslant \eta
$$

I will now apply lemmas 5 and 6. (96) and (101) give us (68), we may therefore by these lemmas extend the definition of the vectors $\left\{h_{j+1 k}(X)\right\}$ to all points $X$ such that $\left|X-X_{i j+1}\right| \leqslant 2 \alpha_{j+1}$ for some $i$; which by (90) includes all $X \in\left(S, \alpha_{j+1}\right)$. Moreover, if every point of the interval $X^{\prime} X^{\prime \prime}$ belongs to $\left(S, \alpha_{j+1}\right)$ and hence lies within $2 \alpha_{j+1}$ of some $X_{i j+1}$ we have by (81)

$$
\left|h_{j+1 k}\left(X^{\prime}\right)-h_{j+1 k}\left(X^{\prime \prime}\right)\right| \leqslant 10 N(N+20) 2^{6 N+41}(8 N)^{N} \eta \frac{\left|X^{\prime}-X^{\prime \prime}\right|}{\alpha_{j+1}},
$$

which by (55)

$$
\leqslant \frac{\delta}{\alpha_{j+1}}\left|X^{\prime}-X^{\prime \prime}\right|
$$

which establishes (91) for $j+1$.

Furthermore, if $X \in S$ there will by $(90)$ be an $X_{i j+1}$ such that

$$
\left|X-X_{i j+1}\right| \leqslant \alpha_{j+1} \text {. }
$$


Let $\Sigma^{*}$ be the plane through $X$ parallel to $\Sigma_{X_{i j+1}}^{j+1}$. Then by (102)

which by (94)

$$
S_{0} S\left(X, \mu \alpha_{j+1}\right) \subset S_{0} S\left(X_{i j+1}, 2 \mu \alpha_{j+1}\right) S\left(X, \mu \alpha_{j+1}\right)
$$

$$
\subset\left(\Sigma_{X_{i j+1}^{j+1}}^{j+2}, 2 \varepsilon \mu \alpha_{j+1}\right) S\left(X, \mu \alpha_{j+1}\right) .
$$

Now $X \in S_{0} S\left(X, \mu \alpha_{j+1}\right)$ and hence this $\subset\left(\Sigma^{*}, 4 \varepsilon \mu \alpha_{j+1}\right) S\left(X, \mu \alpha_{j+1}\right)$, which by Lemma 2 and (91) for $j+1$

$$
\subset\left(\Sigma_{X}^{j+1}, 4 \varepsilon \mu \alpha_{j+1}+N \delta \mu \alpha_{j+1}\right) S\left(X, \mu \alpha_{j+1}\right) .
$$

By (56) this establishes (92) for $j+1$.

On the other hand, by Lemma 2 and (91) for $j+1$

$$
\Sigma_{X}^{j+1} S\left(X, \mu \alpha_{j+1}\right) \subset\left(\Sigma^{*}, N \delta \mu \alpha_{j+1}\right) S\left(X, \mu \alpha_{j+1}\right),
$$

which by the above

$$
\subset\left(\sum_{X_{i j+1}^{j}}^{j+1}, N \delta \mu \alpha_{i+1}+2 \varepsilon \mu \alpha_{j+1}\right) S\left(X, \mu \alpha_{j+1}\right) .
$$

Now $N \delta+2 \varepsilon<1$ and so this

$$
\subset\left(\Sigma_{X_{i j+1}}^{j+1} S\left(X_{i j+1}, 2 \mu \alpha_{j+1}\right), 2 \varepsilon \mu \alpha_{j+1}+N \delta \mu \alpha_{j+1}\right) S\left(X, \mu \alpha_{j+1}\right),
$$

which by (95)

$$
\subset\left(S_{0}, 4 \varepsilon \mu \alpha_{j+1}+N \delta \mu \alpha_{j+1}\right) S\left(X, \mu \alpha_{j+1}\right),
$$

which by (56) establishes (93) for $j+1$; and hence by induction proves the lemma.

LemMa 8. Suppose $S_{0}$ is a bounded set, and $S$ is a subset of $S_{0}$ such that if $X \in S$ and $0<R<R_{0}$ then there will exist a plane ${ }_{R} \Sigma_{X}$ through $X$ such that (85) and (86) hold; and suppose further that there exists a plane $\Sigma$ such that (87) holds, and that $\Sigma_{0}$ is a topological disk contained in $\Sigma$, while $S_{1}, \ldots, S_{j}, \ldots$ are subsets of $S$ such that

$$
\left(S_{1}, \frac{\alpha_{2}}{100}\right) \supset \Sigma_{0}
$$

and

$$
S_{j+1} \supset S_{0}\left(S_{j}, 4 \alpha_{j+1}\right),
$$

where

$$
\frac{\alpha_{j+1}}{\alpha_{j}}=\varrho
$$

Then there will, for each $j$, exist a topological disk $S^{j-1}$ such that $S^{1}=\Sigma_{0}$ and

$$
S^{j-1} \subset\left(S_{j-1}, \frac{\alpha_{j}}{100}\right)
$$

and if

$$
|P Q| \leqslant \frac{1}{20} \alpha_{j}
$$


and $P Q$ belong to $S^{j-1}$ then $P Q$ will make an angle of less than $\frac{1}{2} \theta$ with $\Sigma_{P}^{j-1}$. Moreover, there will be a homeomorphism between $S^{j-1}$ and $S^{j}$ such that if $P, Q \in S^{j-1}$ and $P^{*}, Q^{*}$ are the images in $S^{j}$ of $P, Q$ respectively then, if (106) holds,

$$
\frac{1}{2} \leqslant\left|\frac{P^{*} Q^{*}}{P Q}\right| \leqslant 2
$$

and

$$
\left|P P^{*}\right|<\frac{1}{50} \alpha_{j}
$$

Note first that given any bounded set $S$ we may define a set of points $\left\{X_{i j}\right\}$, for each $j$, to satisfy (89) and (90). For any set of points of $S$ such that (89) holds will have a finite number of members, and if we take the set with the largest possible number of members (90) will hold as well. Thus the conditions of Lemma 7 hold and we may apply the results of that lemma.

In particular if $P \in S^{j-1} \subset\left(S_{j-1}, \alpha_{j} / 100\right)$ then $P$ will belong to $\left(S, \alpha_{j-1}\right)$ so that $\Sigma_{P}^{j-1}$ will be defined.

I will now prove the lemma by induction. Consider first the case $j=2$. If we denote $\Sigma_{0}$ by $S^{1}$ then (105) will become (103); and since $\Sigma_{P}^{1}$ is the plane through $P$ parallel to $\Sigma_{0}$, by Lemma $7, P Q$ will make an angle of less than $\frac{1}{2} \theta$ with $\Sigma_{P}^{1}$ when $P, Q \in S^{1}$. Suppose then that $S^{j-1}$ exists and (105) holds while if $P, Q \in S^{j-1}$ and (106) holds then $P Q$ makes an angle of less than $\frac{1}{2} \theta$ with $\Sigma_{P}^{j-1}$. Suppose

$$
P \in\left(S_{j-1}, \frac{1}{10} \alpha_{j}\right)
$$

Let $\left\{x^{k}(P)\right\}$ be the co-ordinates of the point $X$ referred to axes with origin $P$ and directions $\left\{h_{j k}(P)\right\}$.

Define

$$
\begin{gathered}
f_{i j}^{1}(P)=\sqrt{\sum_{t=1}^{m}\left[x_{i j}^{t}(P)\right]^{2}} \\
f_{i j}^{2}(P)=\left|P X_{i j}\right| \\
f_{i j}(P)=f_{i j}^{1}(P) \text { when }\left|P X_{i j}\right| \leqslant 2 \alpha_{j} \\
f_{i j}(P)=f_{i j}^{1}(P)+f_{i j}^{2}(P)-2 \alpha_{j} \text { when }\left|P X_{i j}\right| \geqslant 2 \alpha_{j} .
\end{gathered}
$$

We can choose an $X_{i j}$, denoted by $X_{0 j}$, such that for all $Q$ such that

$$
|P Q| \leqslant \frac{1}{4} \alpha_{j}
$$

we have

$$
\left|Q X_{i j}\right| \geqslant \frac{1}{4} \alpha_{j}, \quad i \neq 0
$$

and

$$
\left|Q X_{0^{j}}\right|<2 \alpha_{j} \text {. }
$$


For by (89), (90) and (109) if we choose $X_{0^{j}}$ so that

$$
\left|P X_{0^{j}}\right|=\min _{i}\left|P X_{i j}\right| \text {, }
$$

the result will follow. In particular (115) and (116) will hold when $Q=P$.

Suppose

$$
T-1 \leqslant \frac{\left|P X_{i j}\right|}{\alpha_{j}} \leqslant T
$$

Let $N(T)$ be the number of points $X_{i j}$ satisfying (118). By (89) the spheres $S\left(X_{i j}, \frac{1}{2} \alpha_{j}\right)$ are disjoint and contained in $S\left(P,(T+1) \alpha_{j}\right)$ so that

$$
N(T) \leqslant 2^{N}(T+1)^{N} .
$$

I will now prove a number of estimations.

$$
\left[\frac{f_{0_{j}}(P)}{f_{i j}(P)}\right] \leqslant 16 \text { and } f_{i j}(P) \geqslant \frac{1}{8} \alpha_{j}, \quad i \neq 0 .
$$

Suppose first that

$$
\left|P X_{i j}\right| \leqslant\left(\mu-\frac{1}{10}\right) \alpha_{j}, \quad i \neq 0 .
$$

Then by (109) there will exist $X \in S$ such that

and thus

$$
|P X| \leqslant \frac{1}{10} \alpha_{j}
$$

Hence by (92)

$$
\left|X X_{i j}\right| \leqslant \mu \alpha_{j} \text {. }
$$

$X_{i j} \subset\left(\Sigma_{X}^{j}, \xi \alpha_{j}\right)$

There will exist $Y_{i j}$, belonging to the plane through $P$ parallel to $\Sigma_{X}^{j}$, such that

$$
\left|X_{i j} Y_{i j}\right| \leqslant\left(\xi+\frac{1}{10}\right) \alpha_{j}
$$

Now the interval $P X$ lies in $\left(S, \alpha_{j}\right)$. Thus by $(91)$ and Lemma 2

$$
Y_{i j} \subset\left\{\Sigma_{P}^{j}, \frac{N \delta\left|P Y_{i j}\right||P X|}{\alpha_{j}}\right\} .
$$

Hence by (122), (121), (125),

$$
X_{i j} \subset\left\{\Sigma_{P}^{j}, \frac{N \delta}{10}(\mu+\xi) \alpha_{j}+\left(\xi+\frac{1}{10}\right) \alpha_{j}\right\}
$$

so that by (110), (115), (66), (36) and (39),

$$
f_{i j}^{1}(P) \geqslant \frac{1}{4} \alpha_{j}-\frac{N \delta}{10}(\mu+\xi) \alpha_{j}-\left(\xi+\frac{1}{10}\right) \alpha_{j} \geqslant \frac{\alpha_{j}}{8} .
$$


Hence in this case

$$
f_{i j}(P) \geqslant \frac{1}{8} \alpha_{j}
$$

if, on the other hand, $\quad\left|P X_{i j}\right| \geqslant\left(\mu-\frac{1}{10}\right) \alpha_{j}$

then

$$
f_{i j}(P) \geqslant f_{i j}^{2}(P)-2 \alpha_{j} \geqslant\left(\mu-\frac{1}{10}-2\right) \alpha_{j} \geqslant \frac{1}{8} \alpha_{j} \quad \text { also. }
$$

Thus using (116) with $Q=P(120)$ will follow.

$$
\text { If } \quad T \geqslant 5, \frac{f_{0 j}(P)}{f_{i j}(P)} \leqslant \frac{8}{T} .
$$

For, if $T \geqslant 5,2 \alpha_{j} \leqslant \frac{1}{2}(T-1) \alpha_{j} \leqslant \frac{1}{2}\left|P X_{i j}\right|$ so that $f_{i j}(P) \geqslant\left|P X_{i j}\right|-2 \alpha_{j} \geqslant \frac{1}{2}\left|P X_{i j}\right|$ $\geqslant \frac{1}{2}(T-1) \alpha_{j} \geqslant \frac{1}{4}\left(T \alpha_{j}\right)$, which since $f_{0^{j}}(P)<2 \alpha_{j}$ by (116) proves (132).

Let $\phi_{i}^{k}$ be the angle between $X_{i j} X_{0 j}$ and $h_{j k}(P)$. Now $X_{i j} X_{0 j}$ is fixed and hence by (91)

$$
\left|\frac{\partial \phi_{i}^{k}}{\partial P}\right| \leqslant \lim _{P^{\prime} \rightarrow P} \frac{\sin ^{-1}\left\{\delta\left|P^{\prime}-P\right| / 2 \alpha_{j}\right\}}{\left|P^{\prime}-P\right|}=\frac{\delta}{\alpha_{j}} .
$$

Thus by (118) and (116)

$$
\left|x_{i j}^{k}(P)-x_{0 j}^{k}(P)\right|=\left|X_{i j} X_{0 j}\right|\left|\cos \phi_{i}^{k}\right| \leqslant\left|X_{i j} X_{0 j}\right| \leqslant\left|X_{i j} P\right|+\left|P X_{0 j}\right| \leqslant(T+2) \alpha_{j}
$$

and by (118), (116) and (133)

$$
\begin{gathered}
\left|\frac{\partial}{\partial P}\left[x_{i j}^{k}(P)-x_{0 j}^{k}(P)\right]\right|=\left|X_{i j} X_{0 j}\right|\left|\frac{\partial}{\partial P} \cos \phi_{i}^{k}\right| \leqslant(T+2) \alpha_{j} \cdot \frac{\delta}{\alpha_{j}}=(T+2) \delta . \\
\text { If } \quad T \leqslant \mu-\frac{1}{5},\left|x_{i j}^{k}(P)-x_{0 j}^{k}(P)\right| \leqslant 3 \xi \alpha_{j}, \quad k>m .
\end{gathered}
$$

For by (109) there will exist $X$ such that (122), and hence by (118), (123) hold. Then by (92) $X_{i j}$ and $X_{0 j}$ will lie within $\xi \alpha_{j}$ of $\Sigma_{X}^{j}$ and hence the angle between $X_{i j} X_{0 j}$ and its projection on $\Sigma_{X}^{j}$ is less than $\sin ^{-1}\left|2 \xi \alpha_{j} / X_{i j} X_{0 j}\right|$. The angle between any line in $\Sigma_{X}^{j}$ and its projection on $\Sigma_{P}^{j}$ will by (91) and Lemma 2 be less than $\sin ^{-1}\left(N \delta / \alpha_{j}\right)|X P| \leqslant \sin ^{-1}(N \delta / 10)$. Thus the angle between $X_{i j} X_{0 j}$ and $\Sigma_{P}^{j}$ is less than $\sin ^{-1}\left|2 \xi \alpha_{j} / X_{i j} X_{0 j}\right|+\sin ^{-1}(N \delta / 10)$.

Now $k>m$ so that $h_{j k}(P)$ is orthogonal to $\Sigma_{P}^{j}$ and hence

$$
\left|x_{i j}^{k}(P)-x_{0 j}^{k}(P)\right|=\left|X_{i j} X_{0 j}\right|\left|\cos \phi_{i}^{k}\right| \leqslant\left|X_{i j} X_{0 j}\right|\left|\sin \left\{\sin ^{-1} \frac{2 \xi \alpha_{j}}{\left|X_{i j} X_{0 j}\right|}+\sin ^{-1} \frac{N \delta}{10}\right\}\right|
$$




$$
\begin{aligned}
\leqslant\left|X_{i j} X_{0^{j}}\right|\left|\frac{2 \xi \alpha_{j}}{\left|X_{i j} X_{0 j}\right|}+\frac{N \delta}{10}\right| & \leqslant 2 \xi \alpha_{j}+\frac{N \delta}{10}\left\{\left|X_{i j} P\right|+\left|P X_{0^{j}}\right|\right\} \\
& \leqslant 2 \xi \alpha_{j}+\frac{N \delta \mu}{10} \alpha_{j}+\frac{2 N \delta}{10} \alpha_{j}
\end{aligned}
$$

which by (66) gives (136).

$$
\left|\frac{f_{i j}(P) \frac{\partial}{\partial P} f_{0^{j}}(P)-f_{0^{j}}(P) \frac{\partial}{\partial P} f_{i j}(P)}{\left[f_{i j}(P)\right]^{2}}\right| \leqslant \frac{800 N}{\alpha_{j}} .
$$

For let $\psi_{j}(P)$ be the angle between $P X_{0^{j}}$ and $h_{j k}(P)$; then by (91)

$$
\begin{aligned}
\left|\psi_{j}(P)-\psi_{j}\left(P^{\prime}\right)\right| & \leqslant 2 \sin ^{-1} \frac{1}{2} \frac{\delta\left|P P^{\prime}\right|}{\alpha_{j}}+P \hat{X}_{0 j} P^{\prime} \\
& \leqslant 2 \sin ^{-1} \frac{\frac{1}{2}}{\frac{\delta\left|P P^{\prime}\right|}{\alpha_{j}}}+\sin ^{-1} \frac{\left|P P^{\prime}\right|}{\left|P X_{0 j}\right|-\left|P P^{\prime}\right|} .
\end{aligned}
$$

Thus

$$
\left|\frac{\partial}{\partial P} \psi_{j}(P)\right| \leqslant \frac{\delta}{\alpha_{j}}+\frac{1}{\left|P X_{0 j}\right|}
$$

Hence, using (116)

$$
\left|\frac{\partial}{\partial P} x_{0_{j}}^{k}(P)\right| \leqslant\left|\frac{\partial}{\partial P}\right| P X_{0_{j}}\left|\cos \psi_{j}(P)\right| \leqslant 1+2 \delta+1 \leqslant 3 .
$$

Hence by (135)

$$
\left|\frac{\partial}{\partial P} x_{i j}^{c}(P)\right| \leqslant(T+2) \delta+3
$$

so that by (110)

$$
\left|\frac{\partial}{\partial P} f_{i j}^{\prime}(P)\right| \leqslant N T \delta+2 N \delta+3 N
$$

whence by (113) and (111) $\quad\left|\frac{\partial}{\partial P} f_{i j}(P)\right| \leqslant 1+N T \delta+2 N \delta+3 N \leqslant 4 N+N T \delta$.

Hence using (120)

$$
\left|\frac{f_{i j}(P) \frac{\partial}{\partial P} f_{0^{j}}(P)-f_{0^{j}}(P) \frac{\partial}{\partial P} f_{i j}(P)}{\left[f_{i j}(P)\right]^{2}}\right| \leqslant \frac{(4 N+N T \delta)(1+16)}{f_{i j}(P)} .
$$

Now when $T \geqslant \mu+1, f_{i j}(P) \geqslant T \alpha_{j}-2 \alpha_{j} \geqslant \frac{1}{2} T \alpha_{j}$, by (113) and (118), so that

$$
\frac{17(4 N+N T \delta)}{f_{i j}(P)} \leqslant \frac{34(4 N+N T \delta)}{T \alpha_{j}} \leqslant\left\{34 N \delta+\frac{136 N}{\mu}\right\} \frac{1}{\alpha_{j}} \leqslant \frac{800 N}{\alpha_{j}}
$$

while if $T \leqslant \mu+1$, then by $(120),(36),(39),(66)$ 


$$
\frac{17(4 N+N T \delta)}{f_{i j}(P)} \leqslant \frac{17(4 N+N \mu \delta+N \delta) .8}{\alpha_{j}} \leqslant \frac{800 N}{\alpha_{j}}
$$

so that (137) is established in either case.

Let $P^{*}$ be the point whose $k$ th co-ordinate with respect to axes $\left\{h_{j k}(P)\right\}$ at origin $P$ is

Consider $k>m$.

$$
p_{k}^{*}= \begin{cases}\frac{\sum_{i} x_{i j}^{k}(P)\left[f_{i j}(P)\right]^{-4 N}}{\sum_{i}\left[f_{i j}(P)\right]^{-4 N}} & \text { if } k>m \\ 0 & \text { if } k \leqslant m .\end{cases}
$$

$$
\begin{aligned}
& \left|\frac{\partial}{\partial \boldsymbol{P}}\left(p_{k}^{*}-x_{\mathbf{0}_{j}}^{k}(P)\right)\right| \leqslant\left|\frac{\partial}{\partial \boldsymbol{P}} \frac{\sum_{i}\left\{x_{i j}^{k}(P)-x_{0 j}^{k}(P)\right\}\left[\frac{f_{0^{j}}(P)}{f_{i j}(P)}\right]^{4 N}}{\sum_{i}\left[\frac{f_{0^{j}}(P)}{f_{i j}(P)}\right]^{4 N}}\right| \leqslant \\
& \left|\frac{\sum_{i}\left[\frac{f_{0^{j}}(P)}{f_{i j}(P)}\right]^{4 N} \frac{\partial}{\partial P}\left(x_{i j}^{k}(P)-x_{0 j}^{k}(P)\right)}{\sum_{i}\left[\frac{f_{0 j}(P)}{f_{i j}(P)}\right]^{4 N}}\right|+4 N\left|\frac{\sum_{i}\left[\frac{f_{0^{j}}(P)}{f_{i j}(P)}\right]^{4 N-1}\left(x_{i j}^{k}(P)-x_{0 j}^{k}(P)\right) \frac{\partial}{\partial P}\left[\frac{f_{0^{j}}(P)}{f_{i j}(P)}\right]}{\sum_{i}\left[\frac{f_{0 j}(P)}{f_{i j}(P)}\right]^{4 N}}\right| \\
& +4 N\left|\frac{\sum_{i}\left[\frac{f_{0 j}(P)}{f_{i j}(P)}\right]^{4 N-1} \frac{\partial}{\partial P}\left[\frac{f_{0 j}(P)}{f_{i j}(P)}\right]}{\left\{\sum_{i}\left[\frac{f_{0 j}(P)}{f_{i j}(P)}\right]^{4 N}\right\}^{2}} \sum_{i}\left\{x_{i j}^{k}(P)-x_{0 j}^{k}(P)\right\}\left[\frac{f_{0 j}(P)}{f_{i j}(P)}\right]^{4 N}\right| .
\end{aligned}
$$

Now $\sum_{i}\left[\frac{f_{0 j}(P)}{f_{i j}(P)}\right]^{4 N} \geqslant 1$ so that by $(137)$ this less than

$$
\begin{aligned}
\left|\sum_{i}\left[\frac{f_{0_{j}}(P)}{f_{i j}(P)}\right]^{4 N} \frac{\partial}{\partial P}\left\{x_{i j}^{k}(P)-x_{o j}^{k}(P)\right\}\right| & +\frac{3200 N^{2}}{\alpha_{j}}\left|\sum_{i}\left[\frac{f_{0 j}(P)}{f_{i j}(P)}\right]^{4 N-1}\left\{x_{i j}^{k}(P)-x_{0 j}^{k}(P)\right\}\right| \\
& +\frac{3200 N^{2}}{\alpha_{j}}\left|\sum_{i}\left[\frac{f_{0^{j}}(P)}{f_{i j}(P)}\right]^{4 N-1}\right|\left|\sum_{i}\left\{x_{i j}^{k}(P)-x_{0 j}^{k}(P)\right\}\left[\frac{f_{0_{j}}(P)}{f_{i j}(P)}\right]^{4 N}\right|,
\end{aligned}
$$

which by (119), (120), (132), (134), (135) and (136)

$$
\begin{aligned}
\leqslant & \sum_{1 \leqslant T \leqslant A} 16^{4 N} \cdot 2^{N}(T+1)^{N}(T+2) \delta+\sum_{T>A}\left\{\frac{8}{T}\right\}^{4 N} \cdot 2^{N}(T+1)^{N}(T+2) \delta \\
+ & \frac{3200 N^{2}}{\alpha_{j}}\left\{\sum_{1 \leqslant T \leqslant A} 16^{4 N-1} 2^{N}(T+1)^{N} \cdot 3 \xi \alpha_{j}+\sum_{T>A}\left\{\frac{8}{T}\right\}^{4 N-1} 2^{N}(T+1)^{N}(T+2) \alpha_{j}\right\} \\
& \cdot\left\{1+16 \sum_{1 \leqslant T \leqslant A} 16^{4 N-1} 2^{N}(T+1)^{N}+16 \sum_{T>A}\left\{\frac{8}{T}\right\}^{4 N-1} 2^{N}(T+1)^{N}\right\}
\end{aligned}
$$

provided $5<A<\mu-1$. 
This is less than

$$
2^{18 N+2} \delta A_{1}+2^{14 N+2} \delta A_{2}+3200 N^{2}\left\{2^{18 N-2} \xi A_{1}+2^{14 N-2} A_{2}\right\}\left\{1+2^{18 N} A_{1}+2^{14 N+1} A_{2}\right\},
$$

where

$$
A_{\mathbf{1}}=\sum_{1 \leqslant T \leqslant A} T^{N+1} \leqslant A^{N+2}
$$

and

$$
A_{2}=\sum_{T>A} T^{-3 N+2} \leqslant A^{-3 N+4} \sum_{T>A} \frac{1}{(T-1) T} \leqslant 2 A^{-3 N+3} .
$$

Consequently

$$
\begin{aligned}
\mid \frac{\partial}{\partial P}\left[p_{k}^{*}-\right. & \left.x_{o j}^{k}(P)\right] \mid \leqslant 2^{18 N+2} \delta A^{N+2}+2^{14 N+3} \delta A^{-3 N+3} \\
& +3200 N^{2}\left\{2^{18 N-2} \xi A^{N+2}+2^{14 N-1} A^{-3 N+3}\right\}\left\{1+2^{18 N} A^{N+2}+2^{14 N+2} A^{-3 N+3}\right\} \\
& \leqslant 3200 N^{2} 2^{36 N}\left\{\delta A^{N+2}+\xi A^{2 N+4}+A^{-2 N+5}\right\} \\
& \leqslant 3200 N^{2} 2^{36 N}\left\{\delta A^{N+2}+\xi A^{2 N+4}+A^{-1}\right\} \text { since } N \geqslant 3 .
\end{aligned}
$$

Now $5<\xi^{-1 /(4 N+8)}<\mu-1$ by (36), (39), (42) so that we may satisfy (144) by taking $A^{2 N+4}=\xi^{-\frac{1}{2}}$, whence, using (58)

$$
\left|\frac{\partial}{\partial P}\left[p_{k}^{*}-x_{0 j}^{k}(P)\right]\right| \leqslant 3200 N^{2} \cdot 2^{36 N}\left\{\delta \xi^{-\frac{1}{4}}+\xi^{\frac{1}{2}}+\xi^{1 /(4 N+8)}\right\} \leqslant \zeta .
$$

If now $P, Q \in S^{j-1}$ and $|P Q|<\alpha_{j} / 20$ then the whole interval $P Q$ will by (105) be contained in $\left(S_{j-1}, \alpha_{j} / 10\right)$ and by (114) we can use the same $X_{0^{j}}$ for each point of the interval. Thus we may integrate (145) to obtain, for $k>m$,

$$
\left|p_{k}^{*}-x_{0 j}^{k}(P)-q_{k}^{*}+x_{0 j}^{k}(Q)\right| \leqslant \zeta|P Q| \text {. }
$$

Let $X_{0 j}^{P}$ be the projection of $X_{0 j}$ onto $\prod_{P}^{j}$, the plane through $P$ containing $\left\{h_{j k}(P)\right\}$ $k>m$.

$$
\left|X_{0 j}^{P} P^{*}\right| \leqslant N \operatorname{Max}_{k>m} \frac{\sum_{i}\left|x_{i j}^{k_{k}}(P)-x_{0 j}^{k}(P)\right|\left[\frac{f_{0^{j}}(P)}{f_{i j}(P)}\right]^{4 N}}{\sum_{i}\left[\frac{f_{0^{j}}(P)}{f_{i j}(P)}\right]^{4 N}}
$$

which by (119), (120), (132), (134) and (136)

$$
\begin{aligned}
& \leqslant N \sum_{1 \leqslant T \leqslant A} 16^{4 N} \cdot 2^{N}(T+1)^{N} 3 \xi \alpha_{j}+N \sum_{T>A}\left(\frac{8}{T}\right)^{4 N} 2^{N}(T+1)^{N}(T+2) \alpha_{j} \\
& \leqslant 2^{18 N+2} N\left(A_{1} \xi \alpha_{j}+\frac{1}{2} A_{2} \alpha_{j}\right) \\
& \leqslant 2^{18 N+2} N\left(A^{N+2} \xi \alpha_{j}+A^{-3 N+3} \alpha_{j}\right) \leqslant 2^{18 N+2} N\left(A^{N+2} \xi \alpha_{j}+A^{-N-2} \alpha_{j}\right) \\
& \leqslant 2^{18 N+2} N\left(\xi^{3}+\xi^{\frac{1}{4}}\right) \leqslant 2^{18 N+3} N \xi^{\frac{1}{4}} \alpha_{j} .
\end{aligned}
$$


Now we know that $P Q$ makes an angle of less than $\frac{1}{2} \theta$ with $\Sigma_{P}^{j-1}$, by our inductive hypothesis, and there will exist $X \in S$ such that $|P X| \leqslant \alpha_{j} / 100$. Then by (92) and (93)

$$
\begin{aligned}
\Sigma_{X}^{j} S\left(X, \mu \alpha_{j}\right) & \subset\left(S_{0}, \xi \alpha_{j}\right) S\left(X, \mu \alpha_{j}\right) \subset\left(S_{0} S\left(X, \mu \alpha_{j}+\xi \alpha_{j}\right), \xi \alpha_{j}\right) S\left(X, \mu \alpha_{j}\right) \subset \\
& \subset\left(S_{0} S\left(X, \mu \alpha_{j-1}\right), \xi \alpha_{j}\right) S\left(X, \mu \alpha_{j}\right) \subset\left(\Sigma_{X}^{j-1}, 2 \xi \alpha_{j-1}\right) S\left(X, \mu \alpha_{j}\right) .
\end{aligned}
$$

Hence, by (91) and Lemma 2, $Q$ will lie within

$$
|P Q| \sin \frac{1}{2} \theta+\frac{N \delta|P X||P Q|}{\alpha_{j-1}}+\frac{2 \xi \alpha_{j-1}}{\mu \alpha_{j}}|P Q|+\frac{N \delta|P X||P Q|}{\alpha_{j}} \text { of } \Sigma_{P}^{j}
$$

Thus the angle between $P Q$ and $\Sigma_{P}^{j}$ is less than

$$
\sin ^{-1}\left\{\sin \frac{1}{2} \theta+\frac{N \delta|P X|}{\alpha_{j-1}}+\frac{2 \xi \alpha_{j-1}}{\mu \alpha_{j}}+\frac{N \delta|P X|}{\alpha_{j}}\right\} \leqslant \sin ^{-1}\left\{\sin \frac{1}{2} \theta+\frac{2 N \delta}{100}+\frac{2 \xi}{\varrho \mu}\right\},
$$

which by (59) is less than $\theta$. If then $Q^{P}$ is the projection of $Q$ onto $\prod_{P}^{j}$

$$
|P Q| \geqslant\left|Q Q^{P}\right| \geqslant|P Q| \cos \theta \text {. }
$$

Let $X_{0 j}^{Q j}$ be the projection of $X_{0 j}$ onto $\prod_{Q}^{j}$, and $Z$ the projection of $X_{o j}^{Q}$ onto $\prod_{P}^{j}$. Then $Z X_{0 j}^{P}$ will be the projection of $X_{0 j}^{Q} X_{0 j}$ onto $\prod_{P}^{j}$. Now $X_{0 j}^{Q} X_{0 j}$ is perpendicular to $\prod_{Q}^{j}$ and by $(91)$ and Lemma 2 the angle between $Z X_{0 j}^{P}$ and $\prod_{Q}^{j}$ is less than $\sin ^{-1} N \delta|P Q| / \alpha_{j}$; hence

$$
\begin{aligned}
\left|Z X_{0 j}^{P}\right| & \leqslant\left|X_{0 j}^{Q} X_{0 j}\right| \sin \left\{\sin ^{-1} \frac{N \delta|P Q|}{\alpha_{j}}\right\} \leqslant\left|X_{0 j}^{Q} X_{0_{j} j}\right||P Q| \frac{N \delta}{\alpha_{j}} \\
& \leqslant\left|Q X_{0 j}\right||P Q| \frac{N \delta}{\alpha_{j}} \leqslant\left\{\left|P X_{0 j}\right|+|P Q|\right\}|P Q| \frac{N \delta}{\alpha_{j}}
\end{aligned}
$$

which by $(116),(106) \leqslant 3 N \delta|P Q|$.

By (91) and Lemma 2 the distance of $X_{0 j}^{Q}$ from the plane through $Q$ parallel to $\prod_{P}^{j}$ is at most $N\left|Q X_{0 j}^{Q}\right| \cdot \delta|P Q| / \alpha_{j}$ and hence since $\left|Q X_{0 j}\right| \leqslant\left|P X_{0^{j}}\right|+|P Q| \leqslant 3 \alpha_{j}$ we obtain

$$
|| Z X_{0 j}^{Q}|-| Q Q^{P}|| \leqslant 3 N \delta|P Q|
$$

Hence by (148) and (149)

$$
|P Q|+6 N \delta|P Q| \geqslant\left|X_{0 j}^{P} X_{0 j}^{Q}\right| \geqslant|P Q| \cos \theta-6 N \delta|P Q| \text {. }
$$

Let $P^{* *}$ be a point such that $X_{0 j}^{Q} P^{* *}$ and $X_{0 j}^{P} P^{*}$ are equal vectors. Consider two coordinate systems, both with origin $X_{0 j}^{Q}$, the first with axes $\left\{h_{j k}(Q)\right\}$, the second with axes $h_{j k}(P)$. Let $\left\{q_{k}^{* \prime}\right\}$ be the coordinates of $Q^{*}$ in the first system and $\left\{p_{k}^{* * \prime}\right\}$ those of $P^{* *}$ in the second. 
Then

$$
\begin{aligned}
q_{k}^{* \prime} \begin{cases}=q_{k}^{*}-x_{0 j}^{k}(Q) & k>m \\
=0 & k \leqslant m\end{cases} \\
p_{k}^{* *^{\prime}} \begin{cases}=p_{k}^{*}-x_{0 j}^{k}(P) & k>m \\
=0 & k \leqslant m .\end{cases}
\end{aligned}
$$

Let $\left\{p_{k}^{\prime \prime}\right\}$ be the coordinates of $P^{* *}$ in the first system. Then

$$
p_{k}^{\prime \prime}=\sum_{t=1}^{N}\left\{h_{j k}(Q) \cdot h_{j t}(P)\right\} p_{t}^{* * \prime}
$$

Hence $\quad\left|p_{k}^{\prime \prime}-p_{k}^{* *^{\prime}}\right| \leqslant\left|p_{k}^{* *^{\prime}}\left[h_{j k}(Q) \cdot h_{j k}(P)-1\right]+\sum_{t \neq k} p_{t}^{* *^{\prime}}\left[h_{j k}(Q) \cdot h_{j t}(P)\right]\right|$

$$
\leqslant\left|\sum_{t=1}^{N} p_{t}^{* *^{\prime}}\left[h_{j k}(Q) \cdot\left(h_{j t}(P)-h_{j t}(Q)\right)\right]\right|
$$

which by $(91) \leqslant \operatorname{Max}_{t}\left|p_{t}^{* *^{\prime}}\right| \cdot N \delta|P Q| / \alpha_{j}$

$$
\leqslant\left|P^{*} X_{0 j}^{P}\right| \cdot \frac{N \delta|P Q|}{\alpha_{j}},
$$

Thus by (147), (153) and (146)

$$
\left|p_{k}^{\prime \prime}-q_{k}^{* \prime}\right| \leqslant 2^{18 N+3} N^{2} \delta \xi^{\frac{1}{4}}|P Q|+\zeta|P Q|,
$$

so that by (60)

$$
\left|Q^{*} P^{* *}\right| \leqslant 2^{18 N+3} N^{3} \delta \xi^{\frac{1}{4}}|P Q|+N \zeta|P Q| \leqslant \frac{1}{200}|P Q| .
$$

Hence by (151) and the definition of $P^{* *}$

$$
|P Q|\left(1+6 N \delta+\frac{1}{200}\right) \geqslant\left|P^{*} Q^{*}\right| \geqslant|P Q|\left(\cos \theta-6 N \delta-\frac{1}{200}\right),
$$

which by $(61),(46)$ gives

$$
2 \geqslant \frac{\left|P^{*} Q^{*}\right|}{|P Q|} \geqslant \frac{1}{2}
$$

Let $\Sigma^{P}$ be the plane through $X_{0 j}^{P}$ parallel to $\Sigma_{P}^{j}$. This plane contains $X_{0 j}^{P} X_{0 j}$ since it is orthogonal to $\prod_{P}^{j}$. By (105) there will exist $Y_{1} \in S_{j-1}$ such that

$$
\left|P Y_{1}\right|<\frac{\alpha_{j}}{200}
$$

Then since $\left|X_{0 j} Y_{1}\right| \leqslant\left|X_{0 j} P\right|+\left|P Y_{1}\right|<3 \alpha_{j}<\mu \alpha_{j}$, there will exist $Y_{2} \in \Sigma_{X_{0 j}}^{j}$ by (92) such that

$$
\left|Y_{1} Y_{2}\right| \leqslant \xi \alpha_{j}
$$


and since $\left|X_{0 j} Y_{2}\right|<\left|X_{0 j} Y_{1}\right|+\left|Y_{1} Y_{2}\right|<4 \alpha_{j}$, there will exist $Y_{3} \in \Sigma^{P}$ by (91) and Lemma 2 such that, using (116)

$$
\left|Y_{2} Y_{3}\right| \leqslant 4 \alpha_{j} \cdot \frac{N \delta}{\alpha_{j}}\left|P X_{0_{j}}\right| \leqslant 8 N \delta \alpha_{j}
$$

Now $P X_{0 j}^{P}$ is orthogonal to $\sum^{P}$ and hence

$$
\left|P X_{0 j}^{P}\right| \leqslant\left|P Y_{3}\right| \leqslant\left(\frac{1}{100}+\xi+8 N \delta\right) \alpha_{j}
$$

so that by (147) and (62)

$$
\left|P P^{*}\right| \leqslant\left\{\frac{1}{100}+\xi+8 N \delta+2^{18 N+3} N \xi^{\frac{1}{3}}\right\} \alpha_{j} \leqslant \frac{\alpha_{j}}{50} .
$$

We have therefore established the existence of a homeomorphism $P \rightarrow P^{*}$ for $P \in S^{j-1}$ which by (158) and (162) satisfies (107) and (108). Let $S^{j}$ be the image of $S^{j-1}$ under this homeomorphism. Since $X_{0 j} X_{0 j}^{P}$ lies in $\Sigma^{P}, X_{0 j}^{P}$ will, by (91) and Lemma 2, lie within $N\left|X_{0 j} X_{0 j}^{P}\right| \cdot \delta\left|P X_{0^{j}}\right| / \alpha_{j}$ of $\Sigma_{X_{0 j}}^{j}$. Thus since $\left|X_{0 j} X_{0 j}^{P}\right| \leqslant\left|P X_{0^{j}}\right|<2 \alpha_{j}$, we obtain

$$
\left|X_{0 j}^{P} Y_{\mathbf{4}}\right| \leqslant 4 N \delta \alpha_{j}
$$

where $Y_{4}$ is the projection of $X_{0 j}^{P}$ on $\Sigma_{X_{0 j}}^{j}$.

But by (93) $\Sigma_{X_{0 j}}^{j} S\left(X_{0 j}, \mu \alpha_{j}\right) \subset\left(S_{0}, \xi \alpha_{j}\right)$ and $\left|X_{0 j} Y_{4}\right| \leqslant 4 N \delta \alpha+2 \alpha_{j}<\mu \alpha_{j}$ so that there will ba a point $Y_{5} \in S_{0}$ such that

$$
\left|Y_{4} Y_{5}\right|<\xi \alpha_{j} \text {. }
$$

But $\left|Y_{5} P\right| \leqslant(\xi+4 N \delta+2) \alpha_{j}$ so that by (105) $Y_{5} \subset\left(S_{j-1}, 4 \alpha_{j}\right)$ whence by (104) $Y_{5} \in S_{j}$. Thus since by (147), (163), (164), (63) and (64)

$$
\begin{gathered}
\left|P^{*} Y_{5}\right| \leqslant\left(\xi+4 N \delta+2^{18 N+3} N \xi^{\frac{1}{2}}\right) \alpha_{j} \leqslant \frac{\alpha_{j+1}}{100} \\
S^{j} \subset\left(S_{j}, \frac{\alpha_{j+1}}{100}\right) .
\end{gathered}
$$

Suppose now that $\left|P^{*} Q^{*}\right| \leqslant \alpha_{j+1} / 20$. Then by (162), (65) $|P Q| \leqslant \alpha_{j+1} / 20+\alpha_{j} / 25$ $<\alpha_{j} / 20$. Now by (157) and (158) the angle between $P^{*} P^{* *}$ and $P^{*} Q^{*}$ is less than $2 \sin ^{-1}(1 / 100)$. But $P^{*} P^{* *}$ is parallel to $X_{0 j}^{P} X_{0 j}^{Q}$ and by (149) and (151) since $X_{0 j}^{P} \hat{Z} X_{0 j}^{Q}$ is a right angle

$$
X_{0 j}^{P} \hat{X}_{0 j}^{Q} Z \leqslant \sin ^{-1}\left\{\frac{3 N \delta}{\cos \theta-6 N \delta}\right\},
$$


while $Z X_{0 j}^{Q}$ is orthogonal to $\prod_{P}^{j}$ and hence by Lemma 2 and (91) makes an angle of less than $\sin ^{-1}\left\{N \delta\left|P P^{*}\right| / \alpha_{j}\right\}$ with $\Sigma_{P^{*}}^{j}$. Thus using (162) we see that the angle between $P^{*} Q^{*}$ and $\Sigma_{P^{*}}^{j}$ is less than

$$
2 \sin ^{-1}\left(\frac{1}{100}\right)+\sin ^{-1}\left(\frac{3 N \delta}{\cos \theta-6 N \delta}\right)+\sin ^{-1}\left(\frac{N \delta}{50}\right),
$$

which is less than $\frac{1}{2} \theta$ by (36), (44) and (46). This together with (165) completes the induction and proves the lemma.

LEMMA 9. Under the conditions of Lemma 8 there will exist a subset $\bar{S}$ of $S$ and a homeomorphism $P^{1} \rightarrow \bar{P}$ between $\Sigma_{0}$ and $\bar{S}$ such that

$$
\left|P^{1} \bar{P}\right| \leqslant \frac{\alpha_{2}}{40}
$$

Suppose $P^{1} \in S^{1}=\Sigma_{0}$ and define $P^{j} \in S^{j}$ to be the image of $P^{j-1} \in S^{j-1}$ under the homeomorphism between $S^{j}$ and $S^{j-1}$ defined in Lemma 8 .

By (108)

$$
\begin{aligned}
\left|P^{j} P^{j \prime}\right| & \leqslant \frac{1}{50}\left(\alpha_{j+1}+\alpha_{j+2} \ldots\right), \quad j^{\prime} \geqslant j, \\
& \leqslant \frac{\alpha_{j+1}}{50}\left(1+\varrho+\varrho^{2} \ldots\right)
\end{aligned}
$$

which by $(65)$

$$
\leqslant \frac{\alpha_{j+1}}{40}
$$

Now $\alpha_{j} \rightarrow 0$ as $j \rightarrow \infty$ and hence the sequence $P^{j}$ converges to some point $\bar{P}$ such that

$$
\left|P^{j} \bar{P}\right| \leqslant \frac{\alpha_{j+1}}{40}
$$

Moreover, by (105) $\bar{P} \in S$. (167) is a particular case of (169) and it only remains to show that $P^{\prime} \rightarrow \bar{P}$ is a homeomorphism.

\section{Suppose}

$$
\left|P^{1} Q^{1}\right|=d<\frac{1}{20} \alpha_{1}
$$

Choose $j_{0}$ such that

$$
\frac{\alpha_{j_{0}}}{20}>2^{j_{0}-1} d>\frac{\alpha_{j_{6}+1}}{80}
$$

this is possible by (65).

$$
\left|P^{j} Q^{j}\right| \leqslant 2^{j-1} d, \quad j \leqslant j_{0} .
$$

This is true for $j=1$ by (170). Suppose then that

$$
\left|P^{j-1} Q^{j-1}\right| \leqslant 2^{j-2} d
$$


then by (171)

$$
2^{j-2} d<\frac{\alpha_{j_{0}}}{20} \leqslant \frac{\alpha_{j}}{20}
$$

so that by (107)

$$
\left|P^{j} Q^{j}\right| \leqslant 2^{j-1} d
$$

which proves (172) by induction. Thus by (169) and (171)

$$
|\bar{P} \bar{Q}| \leqslant 2^{j_{0}-1} d+\frac{\alpha_{j_{0}+1}}{20} \leqslant \frac{\alpha_{j_{0}}+\alpha_{j_{0}+1}}{20} .
$$

But by (171) $j_{0} \rightarrow \infty$ as $d \rightarrow 0$ so that by (175) $|\bar{P} \bar{Q}| \rightarrow 0$ as $d \rightarrow 0$. If, on the other hand, $\bar{P}=\bar{Q}$, then by (169)

$$
\left|P^{i} Q^{j}\right| \leqslant \frac{\alpha_{j+1}}{20} \text { for all } j
$$

and hence by (107)

$$
\left|P^{j} Q^{j}\right| \geqslant\left|P^{1} Q^{1}\right| \cdot 2^{-j+1}
$$

Thus $\left|P^{1} Q^{1}\right| \leqslant 2^{j-1} \varrho^{j} \alpha_{1} / 20 \leqslant(1 / 20)^{j} \alpha_{1}$ for all $j$ so that $\left|P^{1} Q^{1}\right|=0$, i.e. $P^{1}=Q^{1}$, which completes the proof.

THEOREM. If $S_{0}$ is a bounded set of points in $E_{N}$, and $P$ is a point of $S_{0}$ such that to each $R<R_{0}$ and each $X \in S_{0} S\left(P, R_{0}\right)$ there corresponds a m-plane ${ }_{R} \Sigma_{X}$ through $X$ such that

$$
S_{0} S(X, R) \subset\left({ }_{R} \sum_{X}, \varepsilon R\right) S(X, R)
$$

and

$$
{ }_{R} \Sigma_{X} S(X, R) \subset\left(S_{0}, \varepsilon R\right) S(X, R)
$$

and $\Sigma$ is an m-plane through $P$ such that

$$
\left(\sum, \varepsilon R_{0}\right) \supset S_{0}
$$

Then if $\varepsilon \leqslant 2^{-2000 N^{2}}$ there will exist a topological $m$-disk $\bar{S}$ such that $S_{0} S\left(P,(1 / 16) R_{0}\right)$ $\subset \bar{S} \subset S_{0} S\left(P, R_{0}\right)$.

Take

$$
\begin{gathered}
S=S_{0} S\left(P, R_{0}\right) \\
S_{;}=S_{0} S\left(P, \frac{R_{0}}{2}+\frac{\alpha_{2}}{20}+4 \sum_{t=1}^{j} \alpha_{t}\right) \\
\sum_{0}=\sum S\left(P, \frac{R_{0}}{2}+\frac{\alpha_{2}}{40}\right) .
\end{gathered}
$$

The conditions of Lemma 8 will then be satisfied; for (104) follows at once from (179) and $S_{j} \subset S$ by the definition of $\alpha_{1}, \varrho$; it remains to prove (103). 
By (B) and (C)

Hence by (B)

$$
{ }_{T} \Sigma_{P} S(P, T) \subset\left(S_{0}, \varepsilon T\right) \subset\left(\Sigma, \varepsilon T+\varepsilon R_{0}\right)
$$

$$
\Sigma S(P, T) \subset\left({ }_{T} \Sigma_{P}, \varepsilon T+\varepsilon R_{0}\right) S(P, T) \subset\left(S_{0}, 2 \varepsilon T+\varepsilon R_{0}\right) .
$$

Thus taking $T=R_{0} / 2+\alpha_{2} / 40$ (103) follows from (57).

We may now apply Lemma 9 . There will be a topological disk $\bar{S} \subset S$ which by $(178)$ is contained in $S_{0} S\left(P, R_{0}\right)$. Suppose then that $Q \in S_{0} S\left(P, \frac{1}{16} R_{0}\right)$; note first that by (B), (C), (57) and (180)

$$
\begin{aligned}
& S\left(Q, \frac{1}{8} R_{0}\right)\left(\frac{1}{8} R_{0} \Sigma_{Q}, \frac{1}{8} \varepsilon R_{0}\right) \subset\left(\left(S_{0}, \frac{1}{8} \varepsilon R_{0}\right) S\left(Q, \frac{1}{8} R_{0}\right), \frac{1}{8} \varepsilon R_{0}\right) \\
& \quad \subset\left(\Sigma, \frac{3}{2} \varepsilon R_{0}\right) S\left(Q, \frac{1}{8} R_{0}(1+\varepsilon)\right) \subset\left(\Sigma, \frac{\alpha_{2}}{40}\right) S\left(P, \frac{1}{2} R_{0}\right) \subset\left(\Sigma_{0}, \frac{\alpha_{2}}{40}\right),
\end{aligned}
$$

and that by (B) and (A)

$$
\begin{gathered}
S\left(Q, 2^{-n-1} R_{0}\right)\left(2^{-n-1} R_{0} \Sigma_{Q}, 2^{-n-1} \varepsilon R_{0}\right) \subset\left(\left(S_{0}, 2^{-n-1} \varepsilon R_{0}\right) S\left(Q, 2^{-n-1} R_{0}\right), 2^{-n-1} \varepsilon R_{0}\right) \\
\subset\left(S_{0} S\left(Q, 2^{-n-1} R_{0}(1+\varepsilon)\right), 2^{-n} \varepsilon R_{0}\right) \subset\left({ }^{-n}{ }_{R_{0}} \Sigma_{Q}, 2^{-n+1} \varepsilon R_{0}\right) .
\end{gathered}
$$

By Lemma 9 we have a homeomorphism $P^{1} \leftrightarrow \bar{P}$ between $\Sigma_{0}$ and $\bar{S}$. Define a continuous mapping $P^{2}=\phi\left(P^{1}\right)$ of $\Sigma_{0}$ by:-

$$
P^{2}=\left\{\begin{array}{l}
\bar{P} \text { when } \bar{P} \in S\left(Q, \frac{1}{4} R_{0}\right) \\
P^{1} \text { when } \bar{P} \notin S\left(Q, \frac{3}{8} R_{0}\right) \\
{\left[\frac{|\bar{P} Q|-\frac{1}{4} R_{0}}{\frac{1}{8} R_{0}}\right] P^{1}+\left[\frac{\left.\frac{3}{8} R_{0}-|\bar{P} Q|\right]}{\frac{1}{8} R_{0}}\right] \bar{P} \text { otherwise. }}
\end{array}\right.
$$

When $P^{1}$ belongs to the boundary of $\Sigma_{0}$ then by (180) $P^{1} \notin S\left(Q, 7 R_{0} / 16+\alpha_{2} / 40\right)$ so that by (167) $\bar{P} \notin S\left(Q, 3 R_{0} / 8\right)$ and hence $\phi$ is the identity on the boundary of $\Sigma_{0}$. Moreover, by (167)

$$
\left|\bar{P} P^{2}\right| \leqslant \frac{\alpha_{2}}{40} \text { and }\left|P^{1} P^{2}\right| \leqslant \frac{\alpha_{2}}{40}
$$

and hence

$$
\phi\left(\Sigma_{0}\right) S\left(Q, \frac{1}{8} R_{0}\right) \subset \phi\left(\Sigma_{0}\right) S\left(Q, \frac{1}{4} R_{0}-\frac{\alpha_{2}}{40}\right) \subset \bar{S}
$$

There can be no continuous mapping $\psi_{3}$ which maps $\bar{S} S\left(Q, \frac{1}{8} R_{0}\right)$ onto

$$
s\left(Q, \frac{1}{8} R_{0}\right)\left(\frac{1 R_{0}}{8} \Sigma_{Q}, \frac{1}{8} \varepsilon R_{0}\right)
$$

and is the identity outside $s\left(Q, \frac{1}{8} R_{0}\right)$, where $s(P, r)$ denotes as usual the surface of the sphere. For if there were, $\psi_{3} \phi$ would be the identity on the boundary of $\Sigma_{0}$ and by (183), (184) and (181)

5-60173032. Acta mathematica. 104. Imprimé le 21 septembre 1960 


$$
\begin{aligned}
& \psi_{3} \phi\left(\Sigma_{0}\right) \subset \psi_{3}\left\{\left[\phi\left(\Sigma_{0}\right)-S\left(Q, \frac{1}{8} R_{0}\right)\right]+\phi\left(\Sigma_{0}\right) S\left(Q, \frac{1}{8} R_{0}\right)\right\} \\
& \quad \subset\left(\Sigma_{0}, \frac{\alpha_{2}}{40}\right)-S\left(Q, \frac{1}{8} R_{0}\right) \subset\left(\Sigma_{0}, \frac{\alpha_{2}}{40}\right)-S\left(P, \frac{1}{16} R_{0}\right),
\end{aligned}
$$

which gives a contradiction, since $\Sigma_{0}$ is a disk. In fact I shall now prove by induction that, for $n \geqslant 3$, there can be no continuous mapping $\psi_{n}$ which maps $\bar{S} S\left(Q, 2^{-n} R_{0}\right)$ into $s\left(Q, 2^{-n} R_{0}\right)\left(2^{-n} R_{0} \Sigma_{Q}, 2^{-n} \varepsilon R_{0}\right)$ and is the identity outside $S\left(Q, 2^{-n} R_{0}\right)$.

For suppose $\psi_{n+1}$ did exist. Then by (A) and (182)

$$
\begin{aligned}
\psi_{n+1}\left\{\bar{S} S\left(Q, 2^{-n} R_{0}\right)\right\} & \subset\left[S\left(Q, 2^{-n} R_{0}\right)-S\left(Q, 2^{-n-1} R_{0}\right)\right]\left(2^{-n} R_{0} \Sigma_{Q}, 2^{-n} \varepsilon R_{0}\right) \\
& +s\left(Q, 2^{-n-1} R_{0}\right)\left(2^{-n}{ }_{R_{0}} \Sigma_{Q}, 2^{-n+1} \varepsilon R_{0}\right)
\end{aligned}
$$

and this set can be mapped into $s\left(Q, 2^{-n} R_{0}\right)\left(2^{-n}{ }_{R_{0}} \Sigma_{Q}, 2^{-n} \varepsilon R_{0}\right)$ by a mapping which is the identity outside $S\left(Q, 2^{-n} R_{0}\right)$, so that we can construct $\psi_{n}$; and this proves the result.

In particular then, $\bar{S} S\left(Q, 2^{-n} R_{0}\right)$ cannot be null for any $n \geqslant 3$, which, since $\bar{S}$ is closed, proves that $Q \in \bar{S}$ and so the theorem is established, since $Q$ is any point of $S_{0} S\left(\mathrm{P}, \frac{1}{16} R_{0}\right)$.

\section{Chapter 5}

Throughout this chapter we will take the group $G$ involved in the definition of "Surface" to be the group of integers $\bmod 2$.

Lemma $1^{\prime}$. If $L$ is a simple rectifiable curve with end points $A, B$ and of length $l<r_{0}$, whose projection onto a plane $\Pi$ through $A, B$ lies on a circular arc $T$ of radius $r_{0}$ and centre $P$, and if $C(P, L)$ is a surface of minimum area, then $L$ will be the subarc $A B$ of $T$.

We can, of course, prove this lemma independently but in fact it follows at once from the classical theory. $C(P, L)$ will be a dise of minimum area in the sense of $\Lambda^{2}$ measure and hence also [10] in the sense of Lebesgue area it is therefore [12] a minimal surface in the sense of differential geometry and the result follows at once.

LeMma 2. If $L^{*}$ is a simple closed rectifiable curve on $s(0,1)$ and $C\left(O, L^{*}\right)$ is a surface of minimum area then $L^{*}$ will be a great circle.

By taking the intersection of $C\left(O, L^{*}\right)$ with a narrow right circular cylinder whose axis passes through $O$ orthogonally to $A^{\prime} B^{\prime}$ if follows from the previous lemma that every small subarc $A^{\prime} B^{\prime}$ of $L^{*}$ is a great circle arc and hence the lemma follows.

LEмма 3. If $L^{*}$ is a continuum of finite linear measure on $s(0,1)$ and 


$$
\lim _{r \rightarrow 0} \frac{\Lambda S(Q, r) L^{*}}{2 r} \leqslant 1
$$

for all $Q \in L^{*}$, then $L^{*}$ is either a simple closed Jordan curve or else a simple arc.

By [1, Lemma 4, Chap. VIII] $L^{*}$ is an arcwise connected set. Let $E$ be the set of points $P$ of $L^{*}$ which are interior points of some simple Jordan arc in $L^{*}$. Suppose $P \in E$; let $\Gamma$ be an open arc in $L^{*}$ containing $P$. Clearly $\Gamma \subset E$. If $Q \in \Gamma$ then for some small $r S(Q, r)$ will not contain either end point of $\Gamma$ and

$$
\Lambda S(Q, r) L^{*} \leqslant 9 r / 4
$$

and

$$
\Lambda S(Q, r) \Gamma \geqslant 2 r
$$

Suppose there exists $Q^{\prime} \in S\left(Q, \frac{1}{2} r\right)\left(L^{*}-\Gamma\right)$. There will be an arc $\Gamma^{\prime} \subset L^{*} S(Q, r)$ joining $Q^{\prime}$ to a point on $s(Q, r)$. Thus by (11), (12)

$$
\Lambda\left(\Gamma^{\prime}-\Gamma\right) S(Q, r) \leqslant \frac{1}{4} r,
$$

and

$$
\Lambda \Gamma^{\prime} S(Q, r) \geqslant \frac{1}{2} r \text {. }
$$

Hence $\Gamma^{\prime}$ meets $\Gamma$ in $S(Q, r)$. Let $Q^{\prime \prime}$ be the first intersection of $\Gamma^{\prime}$ with $\Gamma$ counting from $Q^{\prime}$. Then (10) will be contradicted at $Q^{\prime \prime}$. Hence $L^{*} S\left(Q, \frac{1}{2} r\right) \subset \Gamma$. Thus $\Gamma$ is open in $L^{*}$. Let $\Gamma_{P}$ be an open subarc of $\Gamma$ containing $P$ with end points $A_{P}, B_{P} \in E$. Consider $P_{0} \in E$. $L^{*}-\Gamma_{P_{0}}$ will have at most two components. Let $F_{A}$ be the component containing $A_{P_{\mathrm{o}}}$. Then $F_{A}$ will be an arcwise connected set. If $F_{A} \notin E$ there will exist $Q^{\prime} \in F_{A}-E$. Let $\Gamma^{\prime} \subset L^{*}$ be an arc joining $Q^{\prime}$ and $A_{P_{0}}$. Suppose $Q^{\prime \prime} \in F_{A}-\Gamma^{\prime}$. Then there will be an arc $\Gamma^{\prime \prime} \subset F_{A}$ joining $Q^{\prime \prime}$ to a point $\Gamma^{\prime}$. Let $Q^{*}$ be the first intersection of $\Gamma^{\prime \prime}$ with $\Gamma^{\prime}$ counting from $Q^{\prime \prime}$. If $Q^{*}$ is $A_{P_{0}}$ or an interior point of $\Gamma^{\prime}$ we obtain a contradiction with (10) while if $Q^{*}=Q^{\prime}$ then $\Gamma^{\prime}+\Gamma^{\prime \prime}$ is an are in $L^{*}$ containing $Q^{\prime}$ as an interior point so that contrary to hypothesis $Q^{\prime} \in E$. Hence $\Gamma^{\prime}=F_{A}$ so that either $F_{A}$ is a simple are or else $F_{A} \subset E$. In the latter case the $\operatorname{arcs} \Gamma_{P}, P \in F_{A}$ form an open covering of the closed set $F_{A}$ and hence by the Heine-Borel theorem $F_{A}$ is the sum of a finite number of arcs. We may apply the same argument to the other component of $L^{*}-\Gamma_{P_{0}}$ (if any) so that $L^{*}$ is the sum of a finite number of arcs. Now by $(10) L^{*}$ cannot contain three otherwise disjoint arcs meeting in a point and hence the lemma easily follows.

Lемма $4^{\prime}$. If $L$ is a continuum of finitelinear measure, $L \subset s(0,1), k_{1} \in H_{1}(L)$ and the minimum of the area in the class of surfaces with boundary $k_{1}$ is $\frac{1}{2} \Lambda L$ then $L$ is a. great circle. Moreover, if the minimum of the area in the class of all surfaces with 
boundary $\supset H_{1}(L)$ is $\frac{1}{2} \Lambda L$ then $L$ will consist of a finite number of simple arcs meeting only at their end points.

Suppose that $Q \in L$ and that

$$
\lim _{r \rightarrow 0} \frac{\Lambda S(Q, r) L}{r}=k .
$$

Let $t(r)$ be the number of points in $s(Q, r) L$. Then by Lemma 4

$$
\lim _{r \rightarrow 0} \frac{1}{r} \int_{0}^{r} t(x) d x \leqslant k
$$

and hence if $[k]$ is the integral part of $k$ there will exist arbitrarily small $r_{0}$ such that

$$
\int_{0}^{r_{0}} t(x) d x \leqslant r_{0}\left[\frac{1}{2}([k]+1-k)+k\right]
$$

and hence there will exist $r_{1}$ such that

$$
t\left(r_{1}\right) \leqslant \frac{1}{2}([k]+1-k)+k
$$

in which case since $t\left(r_{1}\right)$ is an integer

$$
t\left(r_{1}\right) \leqslant k .
$$

Suppose first that $k \geqslant 4$. Let $P_{i}(i=1,2, \ldots)$ be the points of $s\left(Q, r_{1}\right) L$. If $t\left(r_{1}\right) \leqslant 3$, let $B=\sum_{i} Q P_{i}$ the joins being taken on $s(0,1)$. Then

$$
\Lambda B \leqslant k r_{1}-r_{1}+o\left(r_{1}\right) \text {. }
$$

If $t\left(r_{1}\right) \geqslant 4$, choose the notation so that $\left|P_{1} P_{2}\right|$ minimizes $\left|P_{i} P_{j}\right|$, then $P_{1} \hat{Q P} P_{2} \leqslant \frac{1}{2} \pi$. Let $G$ be the centroid of the spherical triangle $P_{1} Q P_{2}$ and let $B=\overline{G P_{1}}+\overline{G P_{2}}+\overline{G Q}+$ $+\sum_{i>2} \overline{Q P}_{i} . \quad$ Then

$$
\Lambda B \leqslant(k-2) r_{1}+\frac{1}{3}(\sqrt{2}+2 \sqrt{5}) r_{1}+o\left(r_{1}\right) \leqslant k r_{1}-\frac{1}{36} r_{1}+o\left(r_{1}\right) .
$$

In either case $B$ is a continuous image of $\sum \bar{Q} \bar{P}_{i}$ and hence by Lemmas $2 \mathrm{~A}, 6 \mathrm{~A}, 4 \mathrm{~A}$, $13 \mathrm{~A}$ and $11 \mathrm{~A}, C\left(O, B+\overline{L-S\left(Q, r_{1}\right)}\right)+S\left(Q, r_{1}\right) s(0,1)$ is a surface with boundary $\supset H_{1}(L)$. Thus, since $L$ consists of the sum of rectifiable arcs [1], by (13), (14) and (15)

$$
\frac{1}{2} \Lambda L \leqslant \frac{1}{2}\left\{k r_{1}-\frac{1}{36} r_{1}+\Lambda L-k r_{1}+o\left(r_{1}\right)\right\}+\left\{\pi+o\left(r_{1}\right)\right\} r_{1}^{2},
$$

which gives a contradiction when $r_{1}$ is small. Thus $k<4$. Suppose now $k>2$ and consider the two cases of the lemma separately. 
Case 1. Surfaces with boundary $k_{1}$.

Suppose $L s\left(Q, r_{1}\right)=P_{1}+P_{2}+P_{3}$. By Lemma $14 \mathrm{~A}$ we can choose $B, \Lambda B \leqslant 2 r_{1}$, so that by Lemmas $4 \mathrm{~A}, 11 \mathrm{~A}$

$$
C\left(0, B+\overline{L-S\left(\overline{Q, r_{1}}\right)}\right)+S\left(Q, r_{1}\right) s(0,1)
$$

is a surface with boundary $k_{1}$. If $L s\left(Q, r_{1}\right)$ consists of less than three points we can choose $B$ to be their join to achieve the same result. Thus by (13)

$$
\frac{1}{2} \Lambda L \leqslant \frac{1}{2}\left\{2 r_{1}+\Lambda L-k r_{1}+o\left(r_{1}\right)\right\}+\left\{\pi+o\left(r_{1}\right)\right\} r_{1}^{2}
$$

which again gives a contradiction for small $r_{1}$. Thus in this case $k \leqslant 2$ for every $Q \in L$.

Case 2. Surfaces with boundary $\supset H_{1}(L)$.

Let $X$ be the component containing $Q$ of $L S\left(Q, r_{1}\right)$. Xs $\left(Q, r_{1}\right)$ will consist of exactly three points $P_{1}+P_{2}+P_{3}$. For if not then $[7, \S 29$ XIII] we can find two closed disjoint sets $X^{\prime}, X^{\prime \prime}$ such that $X^{\prime}+X^{\prime \prime}=L S\left(Q, r_{1}\right)$ and $X^{\prime} s\left(Q, r_{1}\right)$ consists of at most one point $B_{1} X^{\prime \prime} s\left(Q, r_{1}\right)$ consists of at most two points, whose join I will call $B_{2}$. Then by Lemmas $15 \mathrm{~A}, 11 \mathrm{~A}$ and $4 \mathrm{~A}$ (taking $A_{0}=\overline{L-S}\left(Q, r_{1}\right), A_{1}=X^{\prime}$ and $A_{2}=X^{\prime \prime}$ in $15 \mathrm{~A}$ )

$$
C\left(0, \overline{L-S\left(Q, r_{1}\right)}+B_{1}+B_{2}\right)+S\left(Q, r_{1}\right) s(0,1)
$$

will be a surface with boundary $\supset H_{1}(L)$ whence

$$
\left.\left.\frac{1}{2} \Lambda L \leqslant \frac{1}{2}\left\{\Lambda L+2 r_{1}-k r_{1}+o\left(r_{1}\right)\right\}+\left\{\pi+o\left(r_{1}\right)\right\} r_{1}^{2}\right)\right\},
$$

which gives a contradiction when $k>2$ for small $r_{1}$.

Let now $\lambda$ be the greatest lower bound of $\Lambda Y$ taken over all subcontinua of $L S\left(Q, r_{1}\right)$ containing $P_{1}+P_{2}+P_{3}$. By compactness and lower semicontinuity in the class of continua of bounded linear measure [1, VIII, Theorem 12] $\lambda$ will be attained, in $Y_{0}$ say. $Y_{0}$ will be an arcwise comnected set and hence we can find $R \in Y_{0}$ and three $\operatorname{arcs} P_{1} R=N_{1}, P_{2} R=N_{2}$ and $P_{3} R=N_{s}$ contained in $Y_{0}$ and not meeting except at $R$. ( $R$ could be one of $P_{1}, P_{2}, P_{3}$ in which case the corresponding arc is null.) By the definition of $\lambda, Y_{0}=N_{1}+N_{2}+N_{3}$.

I will now show that $L S\left(Q, r_{1}\right)=N_{1}+N_{2}+N_{3}$. If not $\Lambda L S\left(Q, r_{1}\right)-\lambda=\alpha>0$. $N_{1}+N_{2}+N_{3}$ will divide $S\left(Q, r_{1}\right) s(0,1)$ into three domins $D_{1}, D_{2}, D_{3}$ where the boundary of $D_{i}$ is an arc of $s(Q, r) s(0,1)$ plus $N_{j}+N_{k}, j \neq k \neq i$. Let $\Delta_{1}$ be the complementary domain of $D_{1}-L$ whose boundary contains the arc $P_{2} P_{3}$ of $s\left(Q, r_{1}\right) s(0,1)$. Let $D_{i}^{*}$ be an interior complementary domain in $D_{1}$ of $N_{2}+N_{3}+\overline{\Delta_{1}} . \quad L$ is a continuum of finite linear measure and hence a Peano space. Suppose there were a complementary domain $\Delta^{*}$ of $\overline{D_{i}^{*}}$ in $D_{1}$ other than the one containing $\Delta_{1} . \Delta_{1}, D_{i}^{*}$ and $\Delta^{*}$ are disjoint, with their boundaries in $L$. Moreover, (boundary $\Delta^{*}$ ) $\subset N_{2}+N_{3}+$ (boundary 
$\left.D_{i}^{*}\right) \subset N_{2}+N_{3}+\bar{\Delta}_{1}$, which since $N_{2}+N_{3}$ can not bound a domain means that the boundaries of the three domains have a continuum in common-which is impossible.

Thus the complement of $\overline{D_{i}^{*}}$ in $D_{1}$ is a single domain and the boundary of the domain $\left\{\overline{D_{i}^{*}}\right\}^{0}$ is a simple closed Jordan curve consisting of a subarc $N_{i}^{*}$ (possibly null) of $N_{2}+N_{3}$ together with an arc $M_{i} \subset L-N_{2}-N_{3}$. No two such arcs $M_{i}$ can have more than one point in common.

Now by the definition of $\lambda, \Lambda N_{i}^{*} N_{2} \leqslant \Lambda M_{i}$ and $\Lambda N_{i}^{*} N_{3} \leqslant \Lambda M_{i}$ and hence $\Lambda^{2} D_{i}^{*} \leqslant\left(\pi+o\left(r_{i}\right)\right)\left(3 \Lambda M_{i}\right)^{2}$. Hence for small $r_{i}$

$$
\Lambda^{2}\left(S\left(Q, r_{1}\right)-\Delta_{1}-\Delta_{2}-\Delta_{3}\right) \leqslant 10 \pi\left(\Lambda L S\left(Q, r_{1}\right)-\lambda\right)^{2} \leqslant 10 \pi \alpha^{2} .
$$

We can therefore find a simply connected Jordan domain $\Delta$ in $S\left(Q, r_{1}\right)$ such that $S\left(Q, r_{1}\right) \supset \bar{\Delta} \supset L S\left(Q, r_{1}\right)$ and $\Lambda^{2} \bar{\Delta} \leqslant 11 \pi \alpha^{2}$. Consequently by Lemmas $4 \mathrm{~A}, 13 \mathrm{~A}, 11 \mathrm{~A}$, $C\left(O, L-S\left(Q, r_{1}\right)+N_{1}+N_{2}+N_{3}\right)+\bar{\Delta}$ is a surface with boundary $\supset H_{1}(L)$ and so $\frac{1}{2} \Lambda L \leqslant \frac{1}{2}\left(\Lambda L-\Lambda L S\left(Q, r_{1}\right)+\lambda\right)+11 \pi \alpha^{2}$ whence $1 \leqslant 22 \pi \alpha$. But $\alpha \leqslant \Lambda L S\left(Q, r_{1}\right)$ $\leqslant\left(k+o\left(r_{1}\right)\right) r_{1}$ and hence for small $r_{1}$ we have a contradiction. Thus $L S\left(Q, r_{1}\right)$ $=N_{1}+N_{2}+N_{3}$. The set of points where $k>2$ consists therefore of isolated points and hence of a finite number of points.

If there are no such points then by Lemma $3^{\prime} L$ will be either a simple arc or else a simple closed Jordan curve. But if $L$ were a simple arc then by Lemma $5 \mathrm{~A}$, $H_{1}(L)=0$ and hence by Lemma $1 \mathrm{~A}, L$ would be a surface with boundary $\supset H_{1}(L)$ which since $\Lambda^{2} L=0<\frac{1}{2} \Lambda L$ is not the case. Thus in this case $L$ is a simple closed Jordan curve and hence by Lemma $2^{\prime}$ a great circle. On the other hand, suppose there is a finite set of points $\left\{Q_{j}\right\}$ at which $k>2$. To each such point we can take an open neighbourhood consisting of three arcs $N_{1 i}+N_{2 j}+N_{3 j}$.

$X=L-\sum_{j} N_{1 j}+N_{2 j}+N_{3 j}$ will be a closed set, each component of $X$ will meet at least one $\overline{N_{i j}}$ and hence $X$ has only a finite number of components. By Lemma $3^{\prime}$ each component of $X$ will be either a simple arc or else a simple closed Jordan curve, and the latter leads to a contradiction at the point where it meets an $\overline{N_{i j}}$. Hence the lemma is established.

THEOREM 4. If $S$ is a proper minimal surface with boundary $h_{1}^{*} \in H_{1}(A)$ and $P \in S-A$ then

Let

$$
\varliminf_{r \rightarrow 0} \frac{\Lambda^{2} K(P, r)}{\pi r^{2}} \leqslant 1 .
$$

$$
\alpha=\varliminf_{r \rightarrow 0} \frac{\Lambda^{2} K(P, r)}{\pi r^{2}} .
$$


Suppose there exists $\delta>0$ and $r_{1}>0$ such that for all $r<r_{1}$

$$
\Lambda^{2} K(P, r)<\frac{1}{2} r(1-\delta) \Lambda l(P, r) .
$$

Then, by Lemma 4, for all sufficiently small $r$

$$
\begin{aligned}
\Lambda^{2} K(P, r) & >\int_{0}^{r} \Lambda l(P, t) d t>\int_{0}^{r} \frac{2 \Lambda^{2} K(P, t)}{t(1-\delta)} d t \\
& >\int_{0}^{r} 2 \pi t \alpha\left(1-\frac{1}{2} \delta\right)(1-\delta)^{-1} d t>\alpha\left(1-\frac{1}{2} \delta\right)(1-\delta)^{-1} \pi r^{2}
\end{aligned}
$$

which contradicts (17).

Hence we can find a sequence $r_{n} \rightarrow 0$ such that

$$
\Lambda^{2} K\left(P, r_{n}\right)>\frac{1}{2}\left(1-2^{-n}\right) r_{n} \Lambda l\left(P, r_{n}\right) .
$$

By Lemmas $12 \mathrm{~A}$ and $11 \mathrm{~A}$ there exists $h_{1}^{n} \in H_{1}\left(l\left(P, r_{n}\right)\right)$ such that $K\left(P, r_{n}\right)$ is a proper minimal surface with boundary $h_{1}^{n}$. If follows at once from Lemma $4 \mathrm{~A}$ that $\Lambda^{2} K\left(P, r_{n}\right) \leqslant 4 \pi r_{n}^{2}$.

Suppose now that $C$ is any finite set of components of $l\left(P, r_{n}\right)$. We can find an open set $G_{n}$ such that

and

$$
\begin{aligned}
& C \subset G_{n} l\left(P, r_{n}\right) \subset\left(C, 2^{-n} r_{n}\right) \\
& \Lambda G_{n} l\left(P, r_{n}\right)<\Lambda C+2^{-n} r_{n} .
\end{aligned}
$$

By $[7, \S 29$, XIII] and an application of the Heine-Borel theorem we can find two closed disjoint sets $F_{n}$ and $F_{n}^{*}$ such that

$$
l\left(P, r_{n}\right)=F_{n}+F_{n}^{*} \text { and } C \subset F_{n} \subset G_{n} l\left(P, r_{n}\right) .
$$

By Lemmas $13 \mathrm{~A}$ and $11 \mathrm{~A}$ there will exist $h^{n} \in H_{1}\left(\boldsymbol{F}_{n}\right)$ and $h^{n *} \in H_{1}\left(\boldsymbol{F}_{n}^{*}\right)$ such that if $Y$ and $Y^{*}$ are surfaces with boundary $h^{n}$ and $h^{n *}$ respectively then

$$
\Lambda^{2} Y+\Lambda^{2} Y^{*} \geqslant \Lambda^{2} K\left(P, r_{n}\right) .
$$

Hence by Lemmas 7 and 8 and (19) above

$$
\frac{1}{2}\left(1-2^{-n}\right) r_{n} \Lambda l\left(P, r_{n}\right)<\Lambda^{2} K\left(P, r_{n}\right) \leqslant \frac{1}{2}\left(r_{n} \Lambda F_{n}^{*}\right)+K_{2}^{3}\left\{\Lambda F_{n}\right\}^{2}
$$

Thus either

$$
\Lambda F_{n} \geqslant \frac{r_{n}}{8 K_{2}^{3}} \text { or else } \Lambda F_{n}^{*} \geqslant 2^{n-2} \Lambda F_{n} .
$$

In the former case, by (21), (22), for large $n$, 


$$
\Lambda C>r_{n}\left(\frac{1}{8 K_{2}^{3}}-2^{-n}\right)>\frac{r_{n}}{16 K_{2}^{3}}
$$

and in the latter, using (19)

$$
\begin{aligned}
\Lambda C<\Lambda F_{n} \leqslant 2^{-n+2} \Lambda F_{n}^{*} & \leqslant 2^{-n+2} \Lambda l\left(P, r_{n}\right) \\
& \leqslant 2^{-n+4} \Lambda^{2} K\left(P, r_{n}\right) r_{n}^{-1} \leqslant 2^{-n+4} \cdot 4 \pi r_{n}^{2} r_{n}^{-1} \leqslant 2^{-n+8} r_{n} .
\end{aligned}
$$

Now $C$ is the sum of any finite set of components of $l\left(P, r_{n}\right)$ and hence it follows that $l\left(P, r_{n}\right)$ has at most $256 \pi K_{2}^{3}$ components of measure greater than $r_{n} / 16 K_{2}^{3}$ and that if $C_{n}$ is the sum of these components then

$$
\Lambda\left\{1\left(P, r_{n}\right)-C_{n}\right\} \leqslant 2^{-n+8} r_{n} .
$$

Les us now take $C$ to be $C_{n}$ in the above construction of $F_{n}$ and $F_{n}^{*}$.

Let $A_{n}, A_{n}^{*}$ be the sets on the unit sphere $s(0,1)$ congruent to $F_{n}, F_{n}^{*}$ apart from a change of scale of ratio $r_{n}: 1$ and let $B_{n}$ be the set congruent to $C_{n}$ apart from a similar change in scale. Let $k_{n}, k_{n}^{*}$ be the elements of $H_{1}\left(A_{n}\right), H_{1}\left(A_{n}^{*}\right)$ corresponding to $h_{n}, h_{n}^{*}$ in the obvious way.

Now by [1, VIII, Theorem 12] there will exist a closed set $A$ having a finite number of components and such that there exists a sequence $\left\{n_{i}\right\}$ in which

By (20) and (21)

$$
B_{n_{i}} \rightarrow A \text { and } \Lambda A \leqslant \lim _{i \rightarrow \infty} \Lambda B_{n_{i}} .
$$

$$
A_{n_{i}} \rightarrow A \text { and } \Lambda A \leqslant \lim _{i \rightarrow \infty} \Lambda A_{n_{i}} .
$$

Suppose now that $X$ is any surface with boundary $\supset H_{1}(A)$. Let $D$ be an open set on $s(0,1)$ containing $A$. Then by Lemma $23 \mathrm{~A}$ there will exist $h \in H_{1}(A)$ such that for some arbitrarily large $n_{i}, \bar{D}$ is a surface with boundary $-h+k_{n_{i}}$. Hence by Lemma $11 \mathrm{~A}, X+\bar{D}$ will be a surface with boundary $k_{n_{i}}$. Thus by (23), (26) Lemma 7 and $(19)$

$$
\Lambda^{2}(X+\bar{D}) \geqslant \frac{1}{2}\left(1-2^{-n_{i}}\right) \Lambda A_{n_{i}}-2^{-n_{i}+7} \text {. }
$$

$\Lambda^{2} \bar{D}$ may be chosen small and $n_{i}$ large and hence $\Lambda^{2} X \geqslant \frac{1}{2} \lim _{n_{i \rightarrow \infty}} \Lambda A_{n_{i}}$ which by (27) $\geqslant \frac{1}{2} \Lambda A$.

Consequently by the second half of Lemma $4^{\prime}$ each component of $A$ is topologically a finite simplicial complex and so $H_{1}(A)$ has only a finite number of elements.

Suppose now that we have an infinite sequence of domains $D \supset A$. By Lemma $23 \mathrm{~A}$, to each $D$ there will correspond $h \in H_{1}(A)$ such that $\bar{D}$ is a surface with boundary $-h+k_{n_{i}}$ for some arbitrarily large $n_{i}$. Since there are only a finite number of 
elements in $H_{1}(A)$ we can choose $h_{0}$ to correspond in this manner to an infinity of the domains $D$. If now $Y$ is any surface with boundary $h_{0}$ then by (23), (26), Lemma 7 and (19)

$$
\Lambda^{2}(Y+\bar{D}) \geqslant \frac{1}{2}\left(1-2^{-n_{i}}\right) \Lambda A_{n_{i}}-2^{-n_{i}+7}
$$

Choosing the sequence of domains $D$ so that $\Lambda^{2} \bar{D} \rightarrow 0$ and taking $n_{i}$ large we obtain

which by $(27) \geqslant \frac{1}{2} \Lambda A$.

$$
\Lambda^{2} Y \geqslant \frac{1}{2} \lim _{n_{i} \rightarrow \infty} \Lambda A_{n_{i}}
$$

Hence by the first half of Lemma $4^{\prime}$ each component of $A$ is a great circle. But any two great circles meet, and hence $A$ is a great circle; so that $\Lambda A=2 \Pi$. Then by Lemma $7,(29),(26)$ and the definition of $A_{n}$

$$
\lim _{n_{i \rightarrow \infty}} \frac{\Lambda l\left(P, r_{n_{i}}\right)}{2 \pi r_{n_{i}}} \leqslant 1 .
$$

Thence by the application of Lemma 7 and the minimum area property of $K\left(P, r_{n}\right)$ the theorem follows at once.

Lemia $5^{\prime}$. Suppose $A$ is a closed set, $h \in H_{1}(A), S$ a proper surface of minimum area with boundary $h, X$ a closed subset of $S$ not meeting $A$; then there exists a topological relative manifold $M$ with boundary $\Gamma$ such that

$$
S \supset M \supset M-\Gamma \supset X .
$$

By Theorem 4 and the main theorem of this paper, to each $P \in X$ there corresponds an $r>0$ and a disc $D(P)$ in $S-A$ containing $S S(P, 2 r)$. Let $G(P)$ be the component containing $P$ of $S S^{0}(P, r)$. These form an open covering of $X$ and hence by the Heine-Borel theorem we can find a finite set of spheres $S\left(P_{i}, r_{i}\right)$ such that $\Sigma G\left(P_{i}\right) \supset X$. I shall now construet a set of values $r_{i}<r_{i}^{\prime}<2 r_{i}$ such that

$$
\left.\Lambda S s\left(P_{i}, r_{i}^{\prime}\right)\right)<\infty
$$

$S s\left(P_{i}, r_{i}^{\prime}\right) s\left(P_{j}, r_{j}^{\prime}\right)$ consists of a finite set of points if $i \neq j$

and

$$
S s\left(P_{i}, r_{i}^{\prime}\right) s\left(P_{j}, r_{j}^{\prime}\right) s\left(P_{k}, r_{k}^{\prime}\right)=0 \text { if } i \neq j \neq k .
$$

Since $\Lambda^{2} S<\infty$ we may, by Lemma 4, choose $r_{i}^{\prime}$ to comply with (32). Suppose now that $r_{1}^{\prime}, r_{2}^{\prime}, \ldots, r_{\alpha}^{\prime}$ have been chosen to comply. with $(32),(33),(34)$ for $i, j, k \leqslant \alpha$. Then again by Lemma 4, (32), (33), (34) will hold for $i, j, k \leqslant \alpha+1$ for almost all $r_{\alpha+1}^{\prime}$. Let $D_{i}$ be the set of interior points the set obtained from the closure of the 
component containing $P_{i}$ of $S S^{0}\left(P_{i}, r_{i}^{\prime}\right)$ by adding all interior complementary domains relative to $D\left(P_{i}\right)$.

Then

$$
\begin{gathered}
G\left(P_{i}\right) \subset D_{i} \subset D\left(P_{i}\right) \\
\Gamma_{i}=\bar{D}_{i}-D_{i} \subset S s\left(P_{i}, r_{i}^{\prime}\right) .
\end{gathered}
$$

Thus

$$
\Sigma D_{i} \supset X
$$

$$
\Lambda \Gamma_{i}<\infty
$$

$$
\sum_{i \neq j} \Gamma_{i} \Gamma_{j} \text { consists of a finite set of points }
$$

and

$$
\Gamma_{i} \Gamma_{1} \Gamma_{k}=0 \text { for } i \neq j \neq k \text {. }
$$

Moreover, $\Gamma_{i}$ divides $D\left(P_{i}\right)$ into just two domains $D\left(P_{i}\right)-\bar{D}_{i}$ and $D_{i}$ of both of which it is the total boundary and hence by (38) it is a simple closed Jordan curve and $D_{i}$ is a simple closed Jordan domain. By (39) $\bar{D}_{i} \sum_{j \neq i} \bar{D}_{j}$ will have only a finite number of components.

Let $\left\{Q_{i \beta}\right\}\left(1 \leqslant \beta \leqslant \alpha_{i}\right)$ be those which consist of single points. Each $Q_{i \beta}$ will then be at a positive distance from $\bar{D}_{i} \sum_{j \neq i} \bar{D}_{j}-Q_{i \beta}$ from which it follows that

$$
Q_{i \beta} \notin D_{i}+\sum_{j \neq i} D_{j} .
$$

Hence $Q_{i \beta}$ does not belong to $X$ and is therefore at a positive distance from it. We can therefore find $\alpha_{i}$ disjoint cross cuts $\lambda_{i \beta}$ of $D_{i}$ dividing $D_{i}$ into $D_{i}^{*}$ plus $\alpha_{i}$ domains $D_{i \beta}$ such that

$$
\bar{D}_{i \beta} \cdot\left(X+\sum_{j \neq i} \bar{D}_{j}-Q_{i \beta}\right)=0
$$

and

$$
\bar{D}_{i \beta} \supset Q_{i \beta} \text {. }
$$

Let $\Gamma_{i}^{*}=\bar{D}_{i}^{*}-D_{i}^{*}$, then $\quad \Gamma_{i}^{*} \subset \Gamma_{i}+\sum_{1}^{\alpha_{i}} \lambda_{i \beta}-\sum_{1}^{\alpha_{i}} Q_{i \beta}$

and hence by $(42)$

$$
\Gamma_{i}^{*} \cdot \Gamma_{j}^{*} \subset \Gamma_{i} \cdot \Gamma_{j}-\sum_{1}^{\alpha_{i}} Q_{i \beta}-\sum_{1}^{\alpha_{j}} Q_{j \beta}
$$

By (42)

$$
\Sigma D_{i}^{*} \supset X
$$

and by (45), (39) and (40)

and

$$
\sum_{i \neq j} \Gamma_{i}^{*} \Gamma_{j}^{*} \quad \text { is a finite set of poits }
$$


Suppose now that there were a point $Q$ which is a component of $\bar{D}_{i}^{*} \sum_{j \neq i} \bar{D}_{j}^{*} . Q$ will not be a $Q_{k \beta}$ in virtue of (41), (45) and (40). Let $Y$ be the component containing $Q$ of $\bar{D}_{i} \sum_{j \neq i} \bar{D}_{j}$. By (42)

$$
\bar{D}_{i \beta}\left(Y-Q_{i \beta}\right)=0
$$

and for each $j \neq i$

$$
\bar{D}_{j \beta}\left(Y-Q_{j \beta}\right)=0 .
$$

Hence

$$
Y \subset\left(\bar{D}_{i}^{*}+\sum_{1}^{\alpha_{i}} Q_{i \beta}\right)\left(\sum_{j \neq i}\left(\bar{D}_{j}^{*}+\sum_{1}^{\boldsymbol{\alpha}_{j}} Q_{i \beta}\right)\right)
$$

Hence the points of $Y$ nearer to $Q$ than any of the $Q_{k \beta}$ will lie in $\bar{D}_{i}^{*} \sum_{j \neq i} \bar{D}_{j}^{*}$ and hence $Y=Q$, a single point which contradicts the definition of $Y$.

Let now $M^{0}=\Sigma D_{i}^{*}$. I shall show that $M=\overline{M^{0}}$ is a topological relative manifold. At each point of $M^{0} M$ is locally a dise. $M-M^{0} \subset \Sigma \Gamma_{i}^{*}-\Sigma D_{i}^{*}$. There will thus by (47) and $(48)$ be just two kinds of points in $M-M^{0}$, those belonging to just one $\Gamma_{i}^{*}$, which are edge points of the dise $D_{i}^{*}$, not belonging to any other $D_{j}^{*}$, and points $P$ belonging to two $\Gamma_{i}^{*} ; \Gamma_{1}^{*}$ and $\Gamma_{2}^{*}$ say. $P$ will be at a positive distance from $\sum_{i \neq 1,2} \bar{D}_{i}^{*}$ and hence, since $P$ will not be a component of $\bar{D}_{1}^{*} \sum_{j \neq 1} \bar{D}_{j}^{*}$ it will not be a component of $\bar{D}_{1}^{*} \bar{D}_{2}^{*}$. Thus $\Gamma_{1}^{*}-P$ meets $\bar{D}_{2}^{*}$ near $P$ and vica, versa. Since $\Gamma_{1}^{*}$ and $\Gamma_{2}^{*}$ meet in only a finite number of points $\bar{D}_{1}^{*}+\bar{D}_{2}^{*}$ will be locally a half disc at $P$ with boundary through $P$. Thus $M$ is a half disc at each boundary point which completes the proof.

We are now in a position to complete the proof of Theorems 1 and 2 .

Theorem 1. Take $G$ to be the group of integers modulo 2. Suppose the boundary $A$ consists of the disjoint simple closed Jordan curves $A_{1}, \ldots, A_{\alpha}$. For each $\varepsilon>0$ we can cover each $A_{i}$ by a finite set of spheres of radius less than $\frac{1}{2} \varepsilon$ whose sum forms an open set $M_{i} \supset A_{i}$. We may clearly suppose that the $M_{i}$ are disjoint. Take $X$ to be a closed set in $S-A$ containing $S-\Sigma M_{i}$. We thus, by Lemma $5^{\prime}$, obtain a manifold $M=M(\varepsilon)$ such that

$$
S-A \supset M \supset S-\Sigma M_{i}
$$

By Lemmas $24 \mathrm{~A}, 12 \mathrm{~A}$ and $25 \mathrm{~A}$ the boundary of $M(\varepsilon)$ will tend to $A$ in the sense of Theorem 1 as $\varepsilon \rightarrow 0$ which completes the proof.

Theorem 2. Take $G$ to be the group of real numbers modulo one. By Lemma $20 \mathrm{~A}$ we can find a sequence of subgroups $L_{i}$ of $H_{m-1}(A)$ and a sequence of surfaces $S_{i}$ with boundary $\supset L_{i}$ such that 


$$
\Lambda^{m} S_{i} \rightarrow \underset{S \in g^{*}}{\operatorname{glb}} \Lambda^{m} S
$$

By the main theorem and Lemma 15 we can take each $S_{i}$ to be a proper surface of minimum area.

By $[7 \S 28]$ and Lemma $22 \mathrm{~A}$ the surfaces $S_{i}$ will converge in a subsequence to a set $S$ whose algebraic boundary in $A$ is not zero and hence by Lemma $20 \mathrm{~A}, S \in \mathcal{G}^{*}$. If $P \in S_{i}$ and $A . S(P, r)=0$ then, by the main theorem, $\Lambda^{m} S_{i} S(P, r) \geqslant W_{m} r^{m}$.

Let $S(P, r)$ be any sphere with centre on $S$ not meeting $A$. Then for large $i$ there will exist $P_{i} \in S_{i}$ such that

$$
S\left(P_{i}, r-\varepsilon\right) \subset S(P, r) \text { and hence } \lim _{i \rightarrow \infty} \Lambda^{m} S_{i} S(P, r) \geqslant W_{m} r^{m} .
$$

Thus (compare equations (44)-(48) of Chapter 3) the convergence is lower semicontinuous in area. Hence $S$ has minimum area in $\mathcal{G}^{*}$, and by Lemmas $20 \mathrm{~A}$ and 15 any proper surface of minimum area in $\mathcal{G}^{*}$ will be a proper surface of minimum area with boundary $\supset L$ for some $L \subset H_{m-1}(A)$ whence the rest follows from Lemma $19 \mathrm{~A}$ and the main theorem.

\section{Appendix $(1)$}

Introduction. The main paper, to which this appendix is attached, makes use of some results in algebraic topology; it is the object of this appendix to supply these results. It will be clear to the reader that the formulation of these results has been the result of much collaboration between E. R. Reifenberg and myself; I am happy to acknowledge this collaboration.

The plan of this appendix is as follows. After the introduction comes a definition, defining the concept of a surface; this is the definition used in the main paper. Next come a number of examples and counter-examples, intended to illustrate the definition. Finally there come the results needed for the main paper, numbered as Lemmas $1 \mathrm{~A}$ to $26 \mathrm{~A}$.

We now begin with a short recapitulation. The Plateau problem requires us to find a surface of minimum area with given boundary. To give a solution, then, we must clarify our ideas as to what a surface is, what its area is, and what we mean by a surface with given boundary.

Let us begin by admitting that we shall consider surfaces of $m$ dimensions, lying

(1) By Dr. J. F. Adams, Trinity Hall, Cambridge. 
in Euclidean space of $n$ dimensions; the given boundaries, then, will be $(m-1)$ dimensional. We have next to choose between two alternative points of view, which are both classical in the subject. The one regards a surface as a continuous map (of some standard space); the other regards a surface as a set of points. Throughout the present work, a surface is a point-set; to be precise, a surface is a compact subset $X$ of Euclidean $n$-space $R^{n}$. Further, its area is its Hausdorff measure $\Lambda^{m}(X)$. These choices are made for good reasons, but their justification belongs to the main paper, not to this appendix.

In this appendix, we shall consider the notion of a "surface with given boundary". In the spirit of the choices made above, the boundary will be (at least) a compact subset $A$ of Euclidean $n$-space $R^{n}$; we are particularly interested in the case when $A$ is a (topological) ( $m-1)$-sphere, or some other $(m-1)$-manifold. We shall certainly require that $X$ contains $A$; but this condition is not enough by itself, since we do not wish to admit $A$ as a surface with boundary $A$. We have therefore to impose some condition on $X$ to ensure that it does "span the hole in $A$ ". Now algebraic topology was invented to handle such questions, and we should not shrink from using it.

We propose, then, to define the notion of a "surface with given boundary" in terms of algebraic topology.

Definition of a Surface. We have first to indicate the notions of homology theory which we shall use. The letter $G$ will denote a compact Abelian group of coefficients. The letter $U$ will denote the additive group of real numbers modulo one; the symbol $Z_{2}$ will denote the group of integers modulo two. We are particularly interested in the cases $G=U$ and $G=Z_{2}$. Let $X$ be a compact space, let $A$ be a compact subspace of $X$, and let $m$ be a non-negative integer. Then there is defined the Cech homology group $H_{m}(X, A ; G)$ with coefficients in $G$ (see [6] and [8]). If $A$ is empty, this homology group is written $H_{m}(X ; G)$. Let $Y$ be a second compact space with a compact subspace $B$; and let $f: X \rightarrow Y$ be a continuous map with $f(A) \subset B$. Then there is defined an induced homomorphism

$$
f_{*}: H_{m}(X, A ; G) \rightarrow H_{m}(Y, B ; G)
$$

In particular, if $X \subset Y$ and $A \subset B$, we may make use of the inclusion or injection map defined by $i(x)=x$.

If $X, A$ are as above, then we have inclusion maps

$$
\begin{aligned}
& i_{*}: H_{m}(A ; G) \rightarrow H_{m}(X ; G) \\
& j_{*}: H_{m}(X ; G) \rightarrow H_{m}(X, A ; G) .
\end{aligned}
$$


We have, moreover, a boundary homomorphism

$$
\partial ; H_{m}(X, A ; G) \rightarrow H_{m-1}(A ; G) \quad(\text { for } m>0)
$$

and an augmentation homomorphism

$$
\varepsilon: H_{0}(X ; G) \rightarrow G .
$$

These groups and homomorphisms enjoy various good properties, which may be found in $[6]$.

We can now apply these concepts to the study of surfaces. Let $A$ be a fixed compact subset of $R^{n}$, and let $X$ be another compact subset of $R^{n}$, with $X \supset A$. We may associate with $X$ the kernel $K$ of the homomorphism

$$
i_{*}: H_{m-1}(A ; G) \rightarrow H_{m-1}(X ; G) .
$$

$K$ is thus the set of elements $h$ in $H_{m-1}(A ; G)$ such that $i_{*} h=0$.

Definition. We shall call $K$ the algebraic boundary of $X$. We illustrate this notion at once by examples. We say that $A$ is a retract of $X$ if there is a continuous map $r: X \rightarrow A$ such that $r(a)=a$ for $a \in A$. If $A$ is a retract of $X$, then the algebraic boundary $K$ of $X$ is the zero subgroup; this is immediate, from the following diagram.

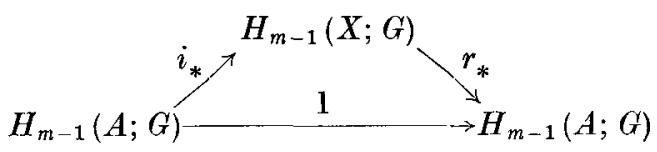

Example 3 is a case in point.

If $X$ is an orientable manifold, as in Example 2, then $K$ is the whole of $H_{m-1}(A ; G)$. In this case, as in the next two examples, the group $H_{m-1}(A ; G)$ is isomorphic to $G$. If $X$ is a non-orientable manifold, as in Example 6, then $K$ consists of the elements of order two in $G$. For example, if $G=U$, then $K$ consists of the residue classes $\{0\},\left\{\frac{1}{2}\right\}$. If $X$ is as in Example 7, then $K$ consists of the elements of order three in $G$. For example, if $G=U$, then $K$ consists of the residue classes $\{0\},\left\{\frac{1}{3}\right\},\left\{\frac{2}{3}\right\}$. Next, let $L$ be a given subgroup of $H_{m-1}(A ; G)$.

Definition. We say that $X$ is a surface with boundary $\supset L$ if the algebraic boundary of $X$ contains $L$. Of course, if we take for $L$ the zero subgroup 0 of $H_{m-1}(A ; G)$, then every set $X$ (containing $A$ ) is a surface with boundary $\supset 0$. This is a trivial ease. For every non-zero subgroup $L$ of $H_{m-1}(A ; G)$, the surfaces with boundary $\supset L$ constitute a significant class of surfaces. Finally, let $h$ be a fixed element of $H_{m-1}(A ; G)$. 
Definition. We say that $X$ is a surface with boundary $\supset h$ if the algebraic boundary of $X$ contains $h$. This is clearly equivalent to saying that $X$ is a surface of boundary $\supset L$, where $L$ is the subgroup of $H_{m-1}(A ; G)$ generated by $h$. It is also equivalent to saying that there exists $k \in H_{m}(X, A ; G)$ with $\partial k=h$; this is immediate, from the following exact sequence.

$$
H_{m}(X, A ; G) \stackrel{\partial}{\rightarrow} H_{m-1}(A ; G) \stackrel{i_{*}}{\rightarrow} H_{m-1}(X ; G)
$$

\section{Examples and Counter-Examples}

Example 1. If $m=2, n=3$ and $X$ is a (topological) disc whose boundary curve is $A$, then we certainly wish $X$ to be a surface with boundary $A$. More generally:-

Example 2. Let $X$ be an orientable (topological) m-manifold-with-boundary whose boundary is the orientable (topological) $(m-1)$-manifold $A$. Then we wish $X$ to be a surface with boundary $A$; and on our definition, it is one.

Example 3. Take $m=2$ and $n=3$, and use complex numbers $z$ to represent points in a fixed plane in $E^{3}$. Let the set $A$ be the circle $|z|=1$, and let the set $X$ be given by

$$
|z| \leqslant 1,\left|z-\frac{1}{2}\right| \geqslant \frac{1}{4},\left|z+\frac{1}{2}\right| \geqslant \frac{1}{4}
$$

so that $X$ is a disc with two holes in it. In this case we do not wish $X$ to be a surface with boundary $A$; and on our definition, it is not.

We remark that, in this example, the boundary $A$ is a retract of $X$, but not a deformation retract of $X$. For this purpose we recall that $A$ is said to be a deformation retract of $X$ if there is a retraction $r: X \rightarrow A$ which is homotopic to the identity map, keeping $A$ fixed. That is, $A$ is a deformation retract of $X$ if there is a continuous map $h: I \times X \rightarrow X$ (where $I=[0,1]$ ) such that

$$
h(0, x)=x \quad h(1, x) \in A \quad h(t, a)=a \text { for } t \in I, a \in A .
$$

Example 4. We take $m=3, n=3$. Let the set $A$ be the torus or ring-surface given in cylindrical polars by

$$
r=1+\varepsilon \mathrm{t} \cos u, \quad \theta=v, \quad z=\varepsilon t \sin u
$$

(where $\varepsilon$ is fixed and small, $t=1,0 \leqslant u \leqslant 2 \pi$, and $0 \leqslant v \leqslant 2 \pi$ ). Let the set $X$ consist of the solid ring (given by $0 \leqslant t \leqslant 1$ ), except that a small open spherical neighbourhood of one internal point is removed. In this case, again, we do not wish $X$ to be a surface with boundary $A$; and on our definition, it is not one. In this example the boundary $A$ is not a retract of $X$. 
Example 5. We take $m=2, n=3$. Let $X$ be the Möbius band given in cylindrical polars by

$$
r=1+\varepsilon t \cos u, \quad \theta=2 u, \quad z=\varepsilon t \sin u
$$

(where $\varepsilon$ is fixed and small, $0 \leqslant t \leqslant 1$, and $0 \leqslant u \leqslant 2 \pi$ ).

The boundary is given by $t=1$, and is a simple closed curve. It is intuitive that if $\varepsilon$ is sufficiently small, there is a minimal surface which approximates closely to the band. This example is a special case of:-

Example 6. Let $X$ be a non-orientable (topological) $m$-manifold-with-boundary whose boundary is the orientable (topological) ( $m-1)$-manifold $A$.

Our definition gives us freedom either to accept this example as a surface, or to reject it. This is done by choice of the coefficient group $G$; if we wish to accept this example as a surface, we take $G=Z_{2}$.

Example 7. The "triple Möbius band". Take $m=2, n=3$, and let $X$ be the set given in cylindrical polars by

$$
r=1+\varepsilon t \cos u, \quad \theta=3 u, \quad z=\varepsilon t \sin u
$$

(where $\varepsilon$ is fixed and small, $0 \leqslant t \leqslant 1$, and $0 \leqslant u \leqslant 2 \pi$ ).

The boundary $A$ is given by $t=1$, and is a simple closed curve. The set $X$ is not a manifold, for it has a singular curve, given by $t=0$, along which three sheets meet at angles of $120^{\circ}$. However, it is intuitive that if $\varepsilon$ is sufficiently small, there is a soap film which approximates closely to the "triple Möbius band". This example is a surface (in the sense of our definition) if $G=U$, but not if $G=Z_{2}$.

Example 8. We take $m=2, n=3$. The set $X$ consists of one copy of Example 5 and one copy of Example 7, joined by a long, thin ribbon of surface. (With the coordinates of Example 5, the axis of the ribbon is given by $r \geqslant 1, \theta=0, z=0$; and where the ribbon meets the band, they have the same tangent planes. The ribbon meets Example 7 similarly.) The boundary $A$ is again a simple closed curve, going (as it were) twice around the Möbius band, along one edge of the ribbon, three times round the triple band, and back along the other edge of the ribbon.

It is intuitive that if the bands and the ribbon are made sufficiently narrow, there is a soap film which approximates closely to this figure. However, this set $X$ is not a surface on our definition, since it admits a retraction onto its boundary $A$. We show this as follows. The set $X$ admits a deformation retraction ( $r^{\prime}$ say) onto a subspace $Y$ consisting of two circles joined by an arc. The space $Y$ admits a map $f: Y \rightarrow A$ such that the circle corresponding to the Möbius band maps with degree 2, 
while the circle corresponding to the triple band maps with degree - 1. By the composite map $f r^{\prime}: X \rightarrow A$, the boundary $A$ maps with degree $2 \cdot 2-1 \cdot 3=1$. Therefore the map $f r^{\prime} \mid A: A \rightarrow A$ is homotopic to the identity. By the homotopy extension theorem, there is a map $r: X \rightarrow A$ such that $r \mid A$ is the identity. Thus $A$ is a retract of $X$.

Of course, it is not easy to visualise the map $r$, since it stretches the narrow width of a band over the whole length of the figure. Similar remarks apply to the next example.

Example 9. We take $m=2, n=3$. We shall construct a set which can clearly be realised as a soap film whose boundary is a closed unknotted curve, but which nevertheless has a deformation retraction onto its boundary, and hence is not a surface on our definition.

We begin by taking a very small regular tetrahedron $A B C D$, with controid $X=(-1,0,0)$, so disposed that $A, B$ lie in the plane $y=0$ with $x>-1$, while $C, D$ lie in the plane $z=0$ with $x<-1$. The point $X$ will be a singular point of our surface. Near $X$, the surface will consist of the six triangles which have $X$ as vertex and a side of the tetrahedron as base.

We next reflect the tetrahedron $A B C D$ in the plane $x=0$, so obtaining a tetrahedron $A^{\prime} B^{\prime} C^{\prime} D^{\prime}$, with centroid $X^{\prime}=(1,0,0)$. The point $X^{\prime}$ will be a singular point of surface similar to $X$.

We next draw a smooth are $\alpha$ in the half-plane $z=0, x \leqslant-1$ which begins with the segment $X C$, ends with the segment $D X$ and encloses a convex dise $\delta$. Reflecting in the plane $x=0$, we obtain a smooth arc $\alpha^{\prime}=X^{\prime} C^{\prime} D^{\prime} X^{\prime}$ enclosing a convex disc $\delta^{\prime}$. Similarly, we draw a simple closed curve $X A A^{\prime} X^{\prime} B^{\prime} B X$ which is smooth except at $X, X^{\prime}$ and lies in the strip $y=0,|x| \leqslant 1$. The ares $\alpha, \alpha^{\prime}, X A A^{\prime} X^{\prime}$ and $X^{\prime} B^{\prime} B X$ will be singular ares on our surface, along which three sheets meet at angles of $120^{\circ}$.

The whole surface consists of the dises $\delta$ and $\delta^{\prime}$, together with a long, narrow, endless ribbon. One edge of this ribbon is the boundary; the other edge is the following are:-

\section{$X A A^{\prime} X^{\prime} D^{\prime} C^{\prime} X^{\prime} A^{\prime} A X D C X A A^{\prime} X^{\prime} B^{\prime} B X C D X B B^{\prime} X^{\prime} C^{\prime} D^{\prime} X^{\prime} B^{\prime} B X$.}

It is understood, of course, that the portion of the ribbon whose edge is said to be $A^{\prime} X^{\prime} D^{\prime}$ contains the triangle $A^{\prime} X^{\prime} D$; similarly for all the other such triangles (except $C X D$ and $C^{\prime} X^{\prime} D^{\prime}$, which lie in the dises $\delta$ and $\delta^{\prime}$ ).

This completes the description of our surface. Let us call it $S$, and its boundary $\Gamma$. If $\Gamma^{\prime}$ is the simple closed curve $X A A^{\prime} X^{\prime} B^{\prime} B X$, then there is clearly a deformation retraction $r^{\prime}: S \rightarrow \Gamma^{\prime}$. The map $r^{\prime}$ is a homotopy equivalence; its inverse is the injection 
$i: \Gamma^{\prime} \rightarrow S$. Let $i: \Gamma \rightarrow S$ be the injection of the boundary. Then $r^{\prime} i: \Gamma \rightarrow \Gamma^{\prime}$ is a map of degree one, and hence a homotopy equivalence. Therefore $i: \Gamma \rightarrow S$ is a homotopy equivalence. The following lemma now shows that $\Gamma$ is a deformation retract of $S$.

L EммA. Suppose that $X$ is a complex, $A$ a subcomplex, and that the injection $i: A \rightarrow X$ is a homotopy equivalence; then $A$ is a deformation retract of $X$.

(I must apologise to specialists in homotopy theory for including a proof they may think unnecessary; I have been asked to do so for the benefit of other readers. The same considerations dictate the use of the proof that follows, rather than the obvious alternative proof by the method of Whitehead's Theorem.)

Proof. We begin by showing that the map $i: A \rightarrow X$ has a homotopy inverse $r: X \rightarrow A$ such that $r i=1_{A}$. In fact, since $i$ is a homotopy equivalence, it has a homotopy inverse $f: X \rightarrow A$. Since $f i \sim 1_{A}$, we have a homotopy $h: I \times A \rightarrow A$ such that

$$
h(0, a)=f(a), h(\mathbf{l}, a)=a .
$$

We may extend $h$ to $h^{\prime}: 0 \times X \cup I \times A \rightarrow A$ by setting $h^{\prime}(0, x)=f(x)$. We can now extend $h^{\prime}$ to $h^{\prime \prime}: I \times X \rightarrow A$ by the homotopy extension theorem. Define $r: X \rightarrow A$ by $r(x)=h(1, a)$; we have $r(a)=a$, that is, $r i=1_{A}$. Moreover, we have $f \sim r: X \rightarrow A$, so ir $\sim$ if $\sim 1_{X}$. We have shown the existence of thr required map $r$.

We will now show that there is a homotopy $h^{\prime}: I \times X \rightarrow X$ such that

$$
h^{\prime}(0, x)=r(x), \quad h^{\prime}(1, x)=x, \quad h^{\prime}(t, a)=a \text { for } a \in A
$$

We may begin by taking a homotopy $h: I \times X \rightarrow X$ such that

$$
h(0, x)=r(x), \quad h(1, x)=x .
$$

We shall now use the homotopy extension theorem on the pair

$$
I \times X, \quad 0 \times X \cup 1 \times X \cup I \times A .
$$

Define a map

$$
k: 0 \times I \times X \cup I \times(0 \times X \cup 1 \times X \cup I \times A) \rightarrow X
$$

by

$$
\begin{array}{ll}
k(0, u, x)=r(x) & k(t, 0, x)=h(t, r(x)) \\
k(t, 1, x)=h(t, x) & k(t, u, a)=h(t, a) .
\end{array}
$$

(We easily verify that these are consistent.) By the homotopy extension theorem, we extend $k$ to $k^{\prime}: I \times I \times X \rightarrow X$, and define $h^{\prime}: I \times X \rightarrow X$ by $h^{\prime}(u, x)=k^{\prime}(1, u, x)$. We have

$$
h^{\prime}(0, x)=r(x), \quad h^{\prime}(1, x)=x, \quad h^{\prime}(u, a)=a .
$$

This completes the proof. 
Results. We now proceed to prove the results which are needed for the main paper. We retain the notations already introduced, but for brevity we omit the symbol for the coefficient group $G$ where no emphasis on it is needed. We make the general convention that the letters $A, B, C, D, X, Y$ and $Z$ (with or without suffixes) shall denote compact spaces; in much of what follows we shall treat them as abstract spaces, since any embedding in $R^{n}$ is irrelevant. The definitions of "algebraic boundary" and "surface" remain meaningful. Since we shall have to deal with a number of inclusion maps, we make the convention that if a space $Y$ contains a subspace $Z$, then $i(Y, Z)$ shall denote the inclusion map from $Z$ to $Y$.

LeMma 1 A. If $X=A$, then the algebraic boundary of $X$ is zero.

This is immediate, because the induced homomorphism $i(X, A)_{*}: H_{m-1}(A) \rightarrow H_{m-1}(X)$ reduces to the identity homomorphism of $H_{m-1}(A)$.

For our next lemma, we recall that $\varepsilon: H_{0}(A) \rightarrow G$ is the augmentation homomorphism.

LEMMA $2 \mathrm{~A}$. If $X$ is contractible, then the algebraic boundary of $X$ is

(i) $H_{m-1}(A)$ if $m>1$

(ii) Ker $\varepsilon$ if $m=\mathbf{1}$.

This lemma will be used to show that contractible "patches" are good for patching holes in surfaces. The result is immediate if $m>1$, since $H_{m-1}(X)=0$ in this case. The case $m=1$ is similar, since $\varepsilon: H_{0}(X) \rightarrow G$ is then an isomorphism.

Lемма 3A. Suppose that $X=\bigcup_{1 \leqslant r \leqslant N} X_{r}$, where the $X_{r}$ are disjoint and contractible. Write $A_{r}=A \cap X_{r}$; write $\varepsilon_{r}: H_{0}\left(A_{r}\right) \rightarrow G$ for the augmentation maps. Define

$$
K_{0}=\sum_{r} i\left(A, A_{r}\right)_{*} \operatorname{Ker} \varepsilon_{r} \subset H_{0}(A) .
$$

Then the algebraic boundary of $X$ is

(i) $H_{m-1}(A)$ if $m>1$

(ii) $K_{0}$ if $m=1$.

The purpose of this lemma is similar to that of Lemma $2 \mathrm{~A}$. The proof is also similar, since $H_{m-1}(X)$ is isomorphic to the direct sum $\sum_{1 \leqslant r \leqslant N} H_{m-1}\left(X_{r}\right)$.

LEMMA 4 A. Suppose that $X$ is a disc; urite $L$ for $H_{m-1}(A)$ if $m>1$, or for Ker $\varepsilon$ if $m=1$. Then $X$ is a surface with boundary $\supset L$.

This is immediate from Lemma $2 \mathrm{~A}$, since a disc is contractible. 
Leмmа $5 \mathrm{~A}$. If $A$ is a Jordan arc, then $H_{1}(A)=0$.

This holds because $A$ is contractible.

LEMMA 6 A. Suppose that $f: X, A \rightarrow Y, B$ is a continuous map. Let $L_{A}$ be a subgroup of $H_{m-1}(A)$; let $L_{B}$ be the subgroup of $H_{m-1}(B)$ consisting of elements of the form $f_{*} h$, where $h \in L_{A}$. Suppose that $X$ is a surface with boundary $\supset L_{A}$. Then $Y$ is a surface with boundary $\supset L_{B}$.

This is immediate by "naturality" ([6], p. 11, Axiom 2).

LEMMA 7 A. Suppose that $L$ is a subgroup of $H_{m-1}(A)$, that $X$ is a surface with boundary $\supset L$, and that $Y \supset X$. Then $Y$ is also a surface with boundary $\supset L$.

This follows immediately from Lemma $6 \mathrm{~A}$, by taking $f$ to be the inclusion map.

LEMMA $8 \mathrm{~A}$. Take $n=m$, and suppose that $A$ is the unit sphere $S^{m-1}$ in $R^{m}$. Then we have tuo cases.

(i) If $X$ contains the unit solid ball $E^{m}$, then the algebraic boundary of $X$ is $H_{m-1}(A)$ if $m>1$, or Ker $\varepsilon$ if $m=1$.

(ii) If $X$ does not contain the unit solid ball $E^{m}$, then the algebraic boundary of $X$ is zero.

Part (i) is immediate from Lemmas $2 \mathrm{~A}$ and $7 \mathrm{~A}$. Part (ii) follows from the fact that $A$ is then a retract of $X$.

Lемма $9 \mathrm{~A}$. Suppose that $X=I \times Y$ and $A=0 \times Y \cup \mathrm{I} \times Y$. Let $i_{0}, i_{1}$ be the obvious embeddings of $Y$ in $A$ as $0 \times Y$ and as $1 \times Y$. Let $K$ be the subgroup of $H_{m-1}(A)$ consisting of elements of the form $i_{1 *} h-i_{0 *} h$, where $h \in H_{m-1}(Y)$. Then the algebraic boundary of $X$ is $K$.

This lemma, like the following one, is intended to allow the use of cylindrical "patches". The result is immediate, by using the homotopy axiom ([6], p. 11, Axiom 5) which is valid for C̆ech homology.

Lемма 10 A. Suppose that $f: I \times Y \rightarrow X$ is a continuous map. Set $A_{0}=f(0 \times Y)$, $A_{1}=f(1 \times Y)$, and $A=A_{0} \cup A_{1}$. Let us write $f_{0}$ for $f \mid 0 \times Y, f_{1}$ for $f \mid 1 \times Y$. Suppose that $f_{0}$ is a homeomorphism from $0 \times Y$ to $A_{0}$, and that we are given a subgroup $L_{0}$ of $H_{m-1}\left(A_{0}\right)$. Let $K$ be the algebraic boundary of $X$. Then we conclude that there is a subgroup $L_{1}$ of $H_{m-1}\left(A_{1}\right)$ such that

$$
K+i\left(A, A_{0}\right)_{*} L_{0}=K+i\left(A, A_{1}\right)_{*} L_{1} .
$$

Proof. For each element $h^{0}$ in $H_{m-1}(0 \times Y)$ we have a corresponding element $h_{1}$ in $H_{m-1}(1 \times Y)$ such that 


$$
i(I \times Y, 0 \times Y)_{*} h^{0}=i(I \times Y, 1 \times Y)_{*} h^{1} .
$$

We have an isomorphism

$$
f_{0 *}: H_{m-1}(0 \times Y) \cong H_{m-1}\left(A_{0}\right) ;
$$

write $L_{0}^{\prime}=f_{0 *}^{-1} L_{0} \subset H_{m-1}(0 \times Y)$; let $L_{1}^{\prime}$ be the corresponding subgroup of $H_{m-1}(1 \times Y)$; define

Then we have

$$
L_{1}=f_{1 *} L_{1}^{\prime} \subset H_{m-1}\left(A_{1}\right) \text {. }
$$

$$
\begin{aligned}
0 & =f_{*} i(I \times Y, 0 \times Y)_{*} h^{0}-f_{*} i(I \times Y, 1 \times Y)_{*} h^{1} \\
& =i(X, A)_{*}\left[i\left(A, A_{0}\right) f_{0 *} h^{\mathbf{0}}-i\left(A, A_{1}\right)_{*} f_{1 *} h^{1}\right] .
\end{aligned}
$$

Thus

$$
i\left(A, A_{0}\right)_{*} f_{0 *} h^{0}-i\left(A, A_{1}\right)_{*} f_{1 *} h^{1} \in K \text {. }
$$

Since $f_{0 *} h^{0}$ may be a general element of $L_{0}$, and $f_{1 *} h^{1}$ may be a general element of $L_{1}$, the required equation follows at once.

LEMMA 11 A. Suppose that $X=\bigcup_{1 \leqslant r \leqslant N} X_{r}$. Suppose given subsets $A_{r} \subset X_{r}$ and $A \subset X$; write $B=A \cup \bigcup_{r} A_{r}$. Let $L_{r}, L$ be subgroups of $H_{m-1}\left(A_{r}\right), H_{m-1}(A)$; suppose that $X_{r}$ is a surface with boundary $\supset L_{r}$. Suppose we have

$$
\text { (Equation E) } \quad i(B, A)_{*} L \subset \sum_{1 \leqslant r \leqslant N} i\left(B, A_{r}\right)_{*} L_{r} .
$$

Then $X$ is a surface with boundary $\supset L$.

This result is needed in order to prove that after cutting holes in a surface, and then patching it again, we still have a surface.

Proof. Suppose that $h \in L$. Then

$$
\begin{aligned}
i(X, A)_{*} h & =i(X, B)_{*} i(B, A)_{*} h \\
& \in i(X, B)_{*} \sum_{1 \leqslant r \leqslant N} i\left(B, A_{r}\right)_{*} L \\
& \in \sum_{1 \leqslant r \leqslant N} i\left(X, X_{r}\right)_{*} i\left(X_{r}, A_{r}\right)_{*} L_{r} \\
& \in \sum_{1 \leqslant r \leqslant N} i\left(X, X_{r}\right)_{*} 0 .
\end{aligned}
$$

Thus $h \in \operatorname{Ker} i(X, A)_{*}$. This concludes the proof.

In applying Lemma $11 \mathrm{~A}$, we require Equation $E$ as data; Lemmas $12 \mathrm{~A}$ to $16 \mathrm{~A}$ are designed to supply this data, in the applications we have in mind.

LEMMA $12 \mathrm{~A}$. With the notations of Lemma $11 \mathrm{~A}$, suppose further that $A \cap X_{r} \subset A_{r}$, 
and that if $r \neq s$, then $X_{r} \cap X_{s} \subset A_{r} \cap A_{s}$. Let $K_{r}$ be the algebraic boundary of $X_{r}$, so that $K_{r} \subset H_{m-1}\left(A_{r}\right)$. Then the algebraic boundary of $X$ is given by

$$
K=i(B, A)_{*}^{-1}\left[\sum_{r} i\left(B, A_{r}\right)_{*} K_{r}\right] .
$$

Proof. By Lemma $11 \mathrm{~A}$ the algebraic boundary of $X$ contains this subgroup; we have to show the converse. That is, we have to show that if $h \in H_{m-1}(A)$ and if $i(X, A)_{*} h=0$, then there exist $h^{r} \in H_{m-1}\left(A_{r}\right)$ such that

$$
i(B, A)_{*} h=\sum_{r} i\left(B, A_{r}\right)_{*} h^{r}
$$

This is proved by diagram-chasing in the following obvious diagram.

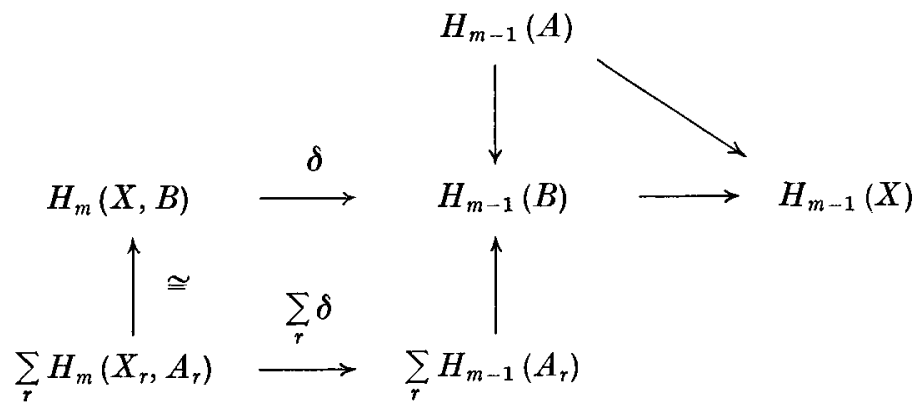

In this diagram, the horizontal line is exact, and the isomorphism marked comes from "excision" (see [6], pp. 11, 266, and cf. p. 33).

Lемма $13 \mathrm{~A}$. Suppose that $A=A_{1} \cup A_{2}, D=A_{1} \cap A_{2}$. Write $K$ for $H_{m-2}(D)$ if $m>2$, or for the kernel of $\varepsilon: H_{0}(D) \rightarrow G$ if $m=2$. Suppose that $B$ is a surface with boundary $\supset K$. Then we have

$i\left(A \cup B, A_{1} \cup B\right)_{*} H_{m-1}\left(A_{1} \cup B\right)+i\left(A \cup B, A_{2} \cup B\right)_{*} H_{m-1}\left(A_{2} \cup B\right) \supset i(A \cup B, A)_{*} H_{m-1}(A)$.

This lemma would allow us to show that the union of two surfaces, one with boundary $A_{1} \cup B$ and the other with boundary $A_{2} \cup B$, is a surface with boundary $A$. We note that the subsidiary surface $B$ is of one less dimension than those we are ultimately concerned with.

The result is proved by diagram-chasing in the following diagram.

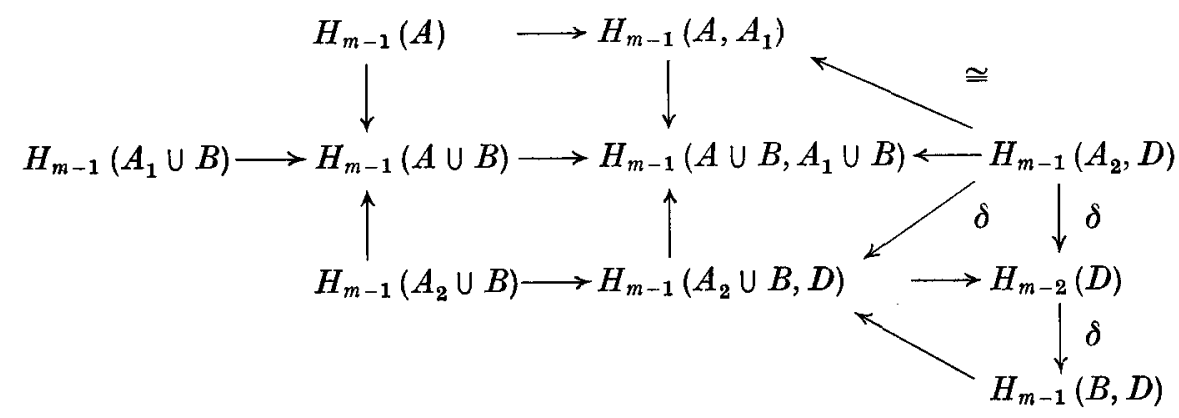


Lemma 14 A. Take $G=Z_{2}, m=2$. Suppose that $A=A_{1} \cup A_{2}$, and that $D=A_{1} \cap A_{2}$ consists of three points. Suppose given $h \in H_{1}(A)$. Then we can choose two of the three points in $D$ so that, if $B$ is an arc joining them, there exist $h^{1} \in H_{1}\left(A_{1} \cup B\right), h^{2} \in$ $H_{1}\left(A_{2} \cup B\right)$ such that $i\left(A \cup B, A_{1} \cup B\right)_{*} h^{1}+i\left(A \cup B, A_{2} \cup B\right)_{*} h^{2}=i(A \cup B, A)_{*} h$.

Proof. Consider the (Mayer-Vietoris) homomorphism

$$
H_{1}(A) \stackrel{j}{\rightarrow} H_{1}\left(A, A_{1}\right) \stackrel{i}{\cong} H_{1}\left(A_{2}, D\right) \stackrel{\partial}{\rightarrow} H_{0}(D) .
$$

Let $k \in H_{1}\left(A_{2}, D\right)$ be an element such that $i k=j h$. Since we have $\varepsilon \partial k=0$ and $H_{0}(D)=Z_{2}+Z_{2}+Z_{2}$, the element $\partial k$ must be one of $(0,0,0),(0,1,1),(1,0,1)$ $(1,1,0)$. We can choose two points of $D$ so that $\partial k$ is zero on the remaining point of $D$. Let $B$ be an arc joining them; then we have an element $L \in H_{1}(B \cup D, D)$ such that $\partial L=\partial k$. The proof is completed in a fashion precisely similar to that of Lemma $13 \mathrm{~A}$, by diagram-chasing in the following diagram.

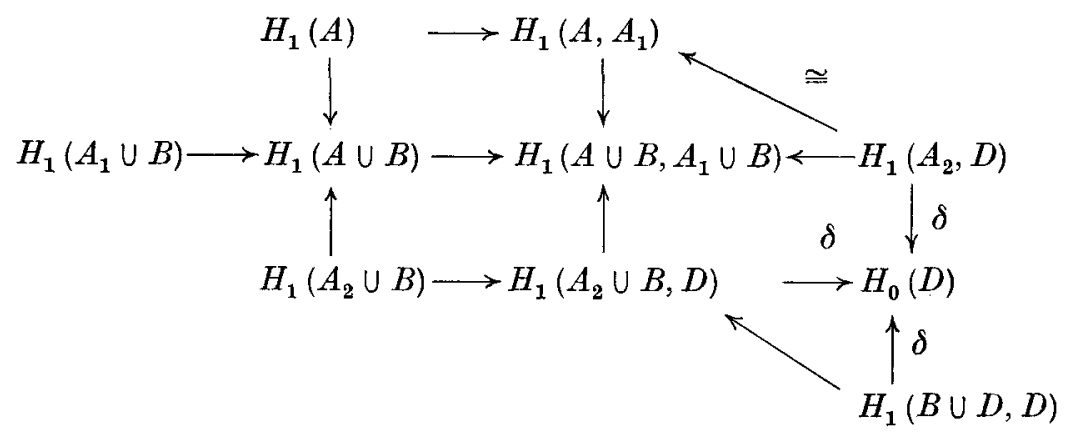

LEMMA 15A. Suppose that

$$
A=A_{0} \cup \underset{1 \leqslant r \leqslant N}{\bigcup} A_{r},
$$

where

$$
\begin{aligned}
& A_{0} \cap A_{r}=D_{r} \text { if } 1 \leqslant r \leqslant N \\
& A_{r} \cap A_{s}=0 \quad \text { if } \quad 1 \leqslant r<s \leqslant N .
\end{aligned}
$$

Write $K_{r}$ for $H_{m-2}\left(D_{r}\right)$ if $m>2$, or for the kernel of $\varepsilon: H_{m-2}\left(D_{r}\right) \rightarrow G$ if $m=2$. Suppose that $B_{r}$ is a surface with boundary $\supset K_{r}$, and write

$$
\begin{aligned}
& C=A \cup \underset{1 \leqslant r \leqslant N}{\cup} B_{r} \\
& C_{0}=A_{0} \cup \bigcup_{1 \leqslant r \leqslant N} B_{r} \\
& C_{r}=A_{r} \cup B_{r} \quad(\text { if } \quad 1 \leqslant r \leqslant N) .
\end{aligned}
$$


Then we conclude that

$$
\sum_{\mathbf{0} \leqslant r \leqslant N} i\left(C, C_{r}\right)_{*} H_{m-1}\left(C_{r}\right) \supset i(C, A)_{*} H_{m-1}(A) .
$$

Proof. We may assume that $B_{N} \cap A=D_{N}$, since the general case follows from this one by a continuous map. The result is now immediate from Lemma $13 \mathrm{~A}$, by induction over $N$.

LEMMA $16 \mathrm{~A}$. Suppose that

$$
A \underset{1 \leqslant r \leqslant N}{\bigcup} A_{r}
$$

where

$$
\begin{aligned}
& A_{r-1} \cap A_{r}=D_{r} \text { if } 1 \leqslant r \leqslant N \\
& A_{r} \cap A_{s}=0 \text { if }|r-s|>1 .
\end{aligned}
$$

Write $K_{r}$ for $H_{m-2}\left(D_{r}\right)$ if $m>2$, or for the kernel of $\varepsilon: H_{0}\left(D_{r}\right) \rightarrow G$ if $m=2$. Suppose that $B_{r}$ is a surface with boundary $\supset K_{r}$ (for $\left.1 \leqslant r \leqslant N\right)$; and set $B_{0}=0, B_{N+1}=0$. Write

Then we conclude that

$$
\begin{aligned}
& C=A \cup \bigcup_{\mathrm{I} \leqslant r \leqslant N} B_{r} \\
& C_{r}=B_{r} \cup A_{r} \cup B_{r+1} \quad(\text { if } 0 \leqslant r \leqslant N) .
\end{aligned}
$$

$$
\sum_{0 \leqslant r \leqslant N} i\left(C, C_{r}\right)_{*} H_{m-1}\left(C_{r}\right) \supset i(C, A)_{*} H_{m-1}(A) .
$$

Proof. We may assume that $B_{N} \cap A=D_{N}$, since that general case follows from this one by a continuous map. The result is now immediate from Lemma $13 \mathrm{~A}$, by induction over $N$.

We now pass on to certain results concerning measure and dimension. We therefore revive the assumption that our spaces are compact subspaces of $R^{n}$, for some $n$.

LemMA $17 \mathrm{~A}$. If $\Lambda^{m-1}(A)=0$, then $H_{m-1}(A)=0$.

Proof. Since $\Lambda_{m-1}(A)=0, \operatorname{dim}(A) \leqslant m-2$, by [8] Theorem VII, 3. This shows that $A$ admits arbitrarily fine coverings whose nerves are simplicial complexes of dimension at most $m-2$. Hence the Cech homology group $H_{m-1}(A)$ is zero (cf. $[8], 151-152)$.

LeмmA $18 \mathrm{~A}$. If $A$ is a totally disconnected set and $m \leqslant 2$, then $H_{m-1}(A)=0$. Since $\operatorname{dim}(A)=0$, this follows in the same way as the preceding lemma.

Lemma $19 \mathrm{~A}$. If $\Lambda^{m}(X-A)=0$ then the algebraic boundary of $X$ is zero.

Proof. Given $\varepsilon>0$ cover $A$ with open sets $O_{i}$ of diameter $<\frac{1}{2} \varepsilon$. Set $O=\bigcup_{i} O_{i}$; write $X^{\prime}=X \cap C O$; then $X^{\prime}$ is compact and $\operatorname{dim}\left(X^{\prime}\right) \leqslant m-1$. Cover $X^{\prime}$ with sets $P_{j}$ 
of diameter $<\frac{1}{2} \varepsilon$ so that not more than $m$ of them have a common point. Now take for a covering of $X$ the sets $O_{i}, P_{j}$, and for a covering of $A$ the sets $O_{i}$ together with those $P_{j}$ which meet on $O_{i}$. Since no such $P_{j}$ is more than $\varepsilon$ from $A$, such pairs of coverings are cofinal in the set of all pairs of coverings. Moreover, when we form the nerve of this pair, all simplexes of dimension $>m-1$ lie in the nerve $N_{A}$. As in Lemma $17 \mathrm{~A}$, we conclude that $H_{m}(X, A)=0$. The required result now follows from the exact sequence

$$
H_{m}(X, A) \stackrel{\partial}{\rightarrow} H_{m-1}(A) \rightarrow H_{m-1}(X)
$$

Lemma $20 \mathrm{~A}$. Tale $G=U$. Suppose that $A$ is a topological $(m-1)$-sphere, that $X \supset A$ and that $\operatorname{dim}(X) \leqslant m$. Then $A$ is a retract of $X$ if and only if the algebraic boundary of $X$ is zero.

We note that the assumption $\operatorname{dim}(X) \leqslant m$ is certainly valid if $\Lambda^{m}(X)<\infty$, by [8], Theorem VII, 3. The result is immediate from Hopf's Extension Theorem as given in [8], p. 147] (Theorem VIII, 1'). We now pass on to three lemmas involving limits.

LEMMA 21 A. Suppose given a set $A$, a fixed subgroup $L$ of $H_{m-1}(A)$, and a decreasing sequence of sets $X_{r} \supset A$, each of which is a surface with boundary $\supset L$. Write $X=\bigcap_{r} X_{r}$. Then $X$ is a surface with boundary $\supset L$.

Proof. Let $h \in L$; then $i\left(X_{r}, A\right)_{*} h=0$, by definition. Now, by the continuity of Cech homology $([6], 260-261)$ the injections $i\left(X_{r}, X\right)$ yield an isomorphism of $H_{m-1}(X)$ with the inverse limit group $\operatorname{Lim} H_{m-1}\left(X_{r}\right)$. This isomorphism maps the element $i(X, A)_{*} h$ into the sequence $\left\{i\left(X_{r}, A\right)_{*} h\right\}$, that is, into the sequence of zeros, which is the zero element of the inverse limit. Thus $i(X, A)_{*} h=0$, and $X$ is a surface with boundary $\supset L$.

LEMma $22 \mathrm{~A}$. Take $G=U$. Suppose given a set $A$ which is a topological $(m-1)$ sphere, and a decreasing sequence of sets $X_{r} \supset A$ such that the algebraic boundary of each $X_{r}$ is not zero. Write $X=\bigcap_{r} X_{r}$. Then the algebraic boundary $K$ of $X$ is not zero

Proof. Let the algebraic boundary of $X_{r}$ be $K_{r}$. It is immediate that $K_{r+1} \subset K_{r}$, so $\left\{K_{r}\right\}$ is a decreasing sequence of subgroups. By the argument used in proving Lemma $21 \mathrm{~A}$, we see that $K=\bigcap_{r} K_{r}$. Now (since we are using Čech homology with compact coefficients) $K_{r}$ is compact. It is also a subgroup of $H_{m-1}(A, U)$, which is isomorphic to $U$. The compact subgroups of $U$ are (i) $U$ itself and (ii) the finite cyclic groups. 
We conclude that the intersection $\bigcap_{r} K_{r}$ is attained after a finite number of steps, that is, $\bigcap_{r} K_{r}=K_{n}$ for some $n$. Hence $K$ is non-zero.

Lемма $23 \mathrm{~A}$. Tale $G=Z_{2}, m=2, n=3$. All the sets considered will lie on the sphere-surface $S^{2}$. Suppose given sets $A, A_{r}, D$ such that $A_{r} \rightarrow A$ and $A \subset \operatorname{Int} D$, where the interior Int $D$ is taken relative to the space $S^{2}$. Suppose given $h^{r} \in H_{1}\left(A_{r}\right)$. Then we conclude, there exists $h \in H_{1}(A)$ such that, for infinitely many $r, D$ is a surface with boundary $\supset$

$$
i\left(A \cup A_{r} A_{r}\right)_{*} h^{r}-i\left(A \cup A_{r}, A\right)_{*} h .
$$

Proof. First, suppose $S^{2}$ triangulated. We may subdivide it so finely that every "closed star" either lies in $D$, or does not meet $A$. Then the closed simplexes, which meet $A$ form a subcomplex $D$, with $A \subset \operatorname{Int} D_{1}, D_{1} \subset D$. The group $H_{1}\left(D_{1}\right)$ is finite.

Next, by repeating the subdivision, we may construct a decreasing sequence of subcomplexes $D_{s}$ such that $A \subset \operatorname{Int} D_{s}$ and $A=\bigcap_{s} D_{s}$. Thus $H_{1}(A)$ is isomorphic to the inverse limit $\operatorname{Lim} H_{1}\left(D_{s}\right)$.

We now argue by limits and finiteness to find a $t$ so large that $i\left(D_{1}, D_{t}\right)_{*} H_{1}\left(D_{t}\right)$ $\left.=i D_{1}, A\right)_{*} H_{1}(A)$. In fact, suppose that $h_{1}$ is a class in $H_{1}\left(D_{1}\right)$ which is in the image $i\left(D, D_{s}\right)_{*} H_{1}\left(D_{s}\right)$ for arbitrarily large $s$. Then $h_{1}$ is of the form $i\left(D_{1}, D_{2}\right)_{*} h_{2}$ for at (most) finitely many $h_{2}$; one of these must be in the image $i\left(D_{2}, D_{s}\right)_{*} H_{1}\left(D_{s}\right)$ for arbitrarily large $n$. Continuing by induction, we obtain a sequence of elements $h_{s} \in H_{1}\left(D_{s}\right)$ with $h_{s-1}=i\left(D_{s-1}, D_{s}\right)_{*} h_{s}$. This sequence defines an element of the inverse limit, and hence of $H_{1}(A)$. We conclude that if $h_{1} \in H_{1}\left(D_{1}\right)$ it not in the image $i\left(D_{1}, A\right)_{*} H_{1}(A)$, then it is not in the image $i\left(D_{1}, D_{s}\right)_{*} H_{1}\left(D_{s}\right)$ for some finite $s$. Since $H_{1}\left(D_{1}\right)$ is finite, we can find at $t$ so large that

$$
i\left(D_{1}, D_{t}\right)_{*} H_{1}\left(D_{t}\right)=i\left(D_{1}, A\right)_{*} H_{1}(A) .
$$

For sufficiently large $r$, we now have $A_{r} \subset D_{t}$. Thus for sufficiently large $r$, we have

$$
i\left(D_{1}, A_{r}\right)_{*} h^{r} \in i\left(D_{1}, A\right)_{*} H_{1}(A) \text {. }
$$

But the elements of $i\left(D_{1}, A\right)_{*} H_{1}(A)$ are finite in number. Hence we can find one of them, say $i\left(D_{1}, A\right)_{*} h$, such that

$$
i\left(D_{1}, A_{r}\right)_{*} h^{r}=i\left(D_{1}, A\right)_{*} h
$$

for an infinity of $n$. By the definition of the algebraic boundary, this completes the proof. 
Lемма 24A. Take $G=Z_{2}, m=2$. Suppose that $A$ is a finite set of disjoint simple closed Jordan curves; let $h \in H_{1}(A)$ be the fundamental class of the manifold $A$. Then any compact 2-manifold-with boundary whose boundary is $A$ is a surface with boundary $\supset h$.

We note that the fundamental class $h$ may be described as follows. Let us write $A=\bigcup_{r} A_{r}$, where the $A_{r}$ are the separate Jordan curves. Then $H_{1}(A) \cong \sum_{r} H_{1}\left(A_{r}\right)$, and $H_{1}\left(A_{r}\right) \cong Z_{2}$. The component of $h$ in each group $H_{1}\left(A_{r}\right)$ is the non-zero element. The lemma is a special case of classical results.

LEMMA $25 \mathrm{~A}$. We take $G=Z_{2}, m=2, n=3$. Suppose given $C \subset S \subset M$, where $C$ is a finite set of disjoint simple closed Jordan curves, $S$ is a compact set, and $M$ is an open subset of $R^{3}$. Let $h \in H_{1}(C)$ be the fundamental class of the manifold $C$. Suppose that $S$ is a surface with boundary $\supset$. Then there exists a compact 2-manifold $R$ with boundary $\Gamma$ and a continuous map $f: R \rightarrow M$ such that $f \mid \Gamma$ maps $\Gamma$ homeomorphically onto $C$.

Proof. We first replace $M$ by a finite complex $N$ in $R^{3}$ such that $S \subset N \subset M$. We now have $i(N, C)_{*} h=0$. Since $C$ and $N$ are finite simplicial complexes, their Čech homology groups and their singular homology groups are isomorphic (see [6]). Thus in singular homology we have $i(N, C)_{*} h^{\prime}=0$, where the element $h^{\prime}$ in singular homology corresponds to $h$ in Čech homology. The equation $i(N, C)_{*} h^{\prime}=0$ means precisely the following. There is a finite set of plane triangles $P_{i} Q_{i} R_{i}$ (which we may suppose disjoint) and continuous maps $f_{i}: P_{i} Q_{i} R_{i} \rightarrow N$ such that the parametrized ares $\left.f_{i}\left(P_{i} Q_{i}\right), f_{i}\left(Q_{i} R_{i}\right), f_{i} P_{i} R_{i}\right)$ have the following properties. Firstly, there is a subset of them which consists of the simple closed Jordan curves of $C$, each repeated once and once only. Secondly, the remaining arcs fall into pairs of equal arcs.

We now construct a quotient space $R$ from the triangles $P_{i} Q_{i} R_{i}$, as follows. If a typical pair of equal arcs is $f_{j}\left|P_{j} Q_{j}, f_{k}\right| Q_{k} R_{k}$, then we indentify corresponding points on the sides $P_{j} Q_{j}$ and $Q_{k} R_{k}$ of the triangles $P_{j} Q_{j} R_{j}$ and $P_{k} Q_{k} R_{k}$. The maps $f_{i}$ pass to the quotient, and define a map $f: R \rightarrow N$. The space $R$ provides the 2-manifold required.

Lемма 26A. Take $G=Z_{2}$. Suppose given $A$ and $h \in H_{m-1}(A)$; let $L$ be the subgroup of $H_{m-1}(A)$ consisting of $h$ and 0 . Then a set $X$ containing $A$ is a surface with boundary $\supset L$ if and only if there exists $k \in H_{m}(X, A)$ such that $\partial k=h$.

As remarked above, this is immediate from the exact sequence

$$
H_{m}(X, A) \stackrel{\partial}{\rightarrow} H_{m-1}(A) \rightarrow H_{m-1}(X) .
$$

This completes the results needed for the main paper. 


\section{References}

[1]. A. S. Besicovitch, On the fundamental geometrical properties of linearly measurable plane sets of points II. Math. Ann., 115 (1938), 295-329.

[2]. - - A general form of the covering principle and relative differentiation of additive functions. Proc. Cambridge Phil. Soc., 41 (1945), 103-110.

[3]. - - Parametric Surfaces I. Compactness. Proc. Cambridge Phit. Soc., 45 (1949), 1-13.

[4]. - - Parametric Surfaces II. Lower Semi-continuity of the Area. Proc. Cambridge Phil. Soc., 45 (1949), 14-23.

[5]. J. Dougras, Minimal Surfaces of Higher Topological Structure. Ann. Math., 40 (1939), 205-298. [This paper contains many illustrations and also an extensive bibliograpy-including most of the author's other papers in this field.]

[6]. S. Eilenberg \& N. E. Steenrod, Foundations of Algebraic Topology. Princeton Mathematical Series No. 15.

[7]. F. Hausdorff, Mengenlehre. 3rd Edition. Dover Publications.

[8]. W. Hurewicz \& H. Wallman, Dimension Theory. Princeton Mathematical Series No. 4.

[9]. E. R. Rentenberg, Parametric Surfaces I. Area. Proc. Cambridge Phil. Soc., 47 (1951), 687-698.

[10]. - - Parametric Surfaces V. Area II. Proc. Lond. Math. Soc., 19 (1955), 342-357.

[11]. —_, Parametric Surfaces II. Tangential Properties. Proc. Cambridge Phil. Soc., 48 (1952), 46-69.

[12]. E. J. McShane, Parametrizations of Saddle Surfaces with Applications to the Problem of Plateau. Trans. Amer. Math. Soc., 35 (1933), 716-733.

Received April 24, 1959. 